\title{
Rotating jet phenomena in Active Galactic Nuclei
}

\author{
Dissertation \\ zur Erlangung des Doktorgrades \\ der Mathematisch-Naturwissenschaftlichen \\ Fakultäten \\ der Georg-August Universität zu Göttingen
}

vorgelegt von

Frank Michael Rieger

aus Pforzheim

Göttingen 2000 
D 7

Referent: PD Dr. K. Mannheim

Korreferent: Prof. Dr. K. Beuermann

Tag der mündlichen Prüfung: 01.02.2001 
Wir erkennen die Wahrheit nicht nur mit der Vernunft, sondern auch mit dem Herzen.

Nous connaissons la vérité, non seulement par la raison, mais encore par le cœur.

Blaise Pascal, Pensée 



\begin{abstract}
Frank M. Rieger:

Rotating jet phenomena in Active Galactic Nuclei

Highly collimated and often bipolar outflows have been observed in many Active Galactic Nuclei (AGN). The present thesis considers in detail the implications of an intrinsic and extrinsic rotation in such jets:

The first part deals with the centrifugal acceleration of charged test particles at the base of an intrinsically rotating jet magnetosphere. The equation characterizing the radial accelerated motion is derived and an analytical solution presented. For electrons moving outwards along rotating magnetic field lines, the maximum attainable Lorentz factor is found to be limited to a few hundred by (i) inverseCompton losses in the disk radiation field and (ii) the breakdown of the beadon-the-wire approximation which occurs in the vicinity of the light cylinder. The results show that this mechanism may not only provide pre-accelerated seed particles required for efficient Fermi-type particle acceleration at larger scales, but could also contributes to the hard X-ray hump observed in AGN.

The second part extends the investigation of the previous chapter. Following previous work by Webb (1989), steady-state Green's solutions of the kinetic equations describing the transport of energetic particles in a collisionless, rotating background flow are discussed for conditions assumed to prevail in the jets of active galactic nuclei. By considering rigid and keplerian rotation profiles the centrifugal and shear acceleration of particles scattered by magnetic inhomogeneities are distinguished. In the case of rigidly rotating background flows shearing is absent and the energy gain is analogous to the bead-on-the-wire approach of part 1. For keplerian rotation profiles both shear and centrifugal effects are present. In the case where the shear effects dominate, it is confirmed that power-law particle momentum solutions exist if the mean scattering time is an increasing function of momentum. The relevance of shear acceleration in addition to Fermi-type particle acceleration in AGN jets is finally pointed out with reference to recent observations (e.g. in $3 \mathrm{C} 273)$.

The final part considers the influence of an extrinsic caused jet rotation in the case of the well-kown AGN Mkn 501. It has been shown for the first time that the TeV and X-ray periodicity of $\sim 23$ days, observed in 1997, could be basically interpreted as a doppler-shifted flux modulation due to the orbital motion of the relativistic jet in a supermassive binary black hole system. Using typical jet parameters the intrinsic orbital period and the centre-of-mass distance have been determined, indicating a very close binary system. A simple upper limit on the allowed binary masses has been obtained assuming that the current binary separation is of such an order that gravitational radiation might become dominant, yielding black hole masses which interestingly agree with expectations from merger scenarios.
\end{abstract}





\section{Contents}

1 Basic properties and the physics of AGN 1

1.1 Taxonomy of AGN . . . . . . . . . . . . . . . . . . 1

1.2 Black hole - accretion disk paradigm . . . . . . . . . . . . . 4

1.3 Superluminal motion and flux enhancement in relativistic jets 6

1.4 Unified models . . . . . . . . . . . . . . . . . . . . . . . . 9

1.5 The formation and collimation of jets . . . . . . . . . . . . 9

1.6 High energy emission and particle acceleration in AGN . . . . 13

1.7 The concept of this work . . . . . . . . . . . . . . . 15

$\begin{array}{llll}2 & \text { Test particle acceleration by rotating jet magnetospheres } & 17\end{array}$

2.1 Introduction . . . . . . . . . . . . . . . . . . . . 17

2.2 On centrifugal acceleration in AGN $\ldots \ldots \ldots \ldots$

2.3 Analysis of forces in a rotating reference frame . . . . . . . . . 19

2.4 Analytic solution for the radial acceleration . . . . . . . . . 21

2.5 Results for the maximum Lorentz factor . . . . . . . . . . 25

2.6 Discussion . . . . . . . . . . . . . . . . . . . . . . . . . . . . . 31

3 Rotating and shearing jet flows 35

3.1 Introduction . . . . . . . . . . . . . . . . . . 35

3.2 Relativistic transport theory . . . . . . . . . . . . . . . . 37

3.3 The steady state transport equation in the diffusion approximation . . . . . . . . . . . . . . . . 39

3.4 Transport equation for cylindrical coordinates . . . . . . . . . 40

3.5 Green's function solutions . . . . . . . . . . . . . . . . . 46 
3.5.1 Fourier method and the Green's functions for infinite domains ................... 46

3.5.2 On the method of images and the Green's functions for bounded domains . . . . . . . . . . . . . 47

3.5.3 General Green's formula for Dirichlet boundary conditions . . . . . . . . . . . . . . 49

3.5.4 One- and two-dimensional Green's tunctions . . . . . . 50

3.6 Particle acceleration by rigidly rotating flows . . . . . . . . . . 52

3.6.1 Solution of the transport equation . . . . . . . . . . . . 54

3.6 .2 Results and discussion . . . . . . . . . . . . . . 57

3.7 Acceleration by keplerian rotating flows . . . . . . . . . . . . . 60

3.7 .1 Solution of the transport equation . . . . . . . . . . . . 62

3.7 .2 Results and discussion . . . . . . . . . . . . . . . 67

3.8 Conclusion . . . . . . . . . . . . . . . . . . . . . . 69

4 Rotating jets in binary black holes 73

4.1 Introduction . . . . . . . . . . . . . . . . . . . . . 73

4.1 .1 Evolution of binary black hole systems . . . . . . . . . 74

4.1 .2 Evidence for binary black holes . . . . . . . . . . . . . 75

4.2 The gamma-ray blazar Mkn 501 . . . . . . . . . . . . . . . . 77

4.3 Possible evidence for a binary system in Mkn 501 . . . . . . . 79

4.3 .1 Doppler-shifted flux modulation . . . . . . . . . . . . 79

$4.3 .2 \quad$ Upper limits for the binary masses . . . . . . . . . . . 84

4.4 Discussion . . . . . . . . . . . . . . . . . . . . . . . . . 87

$\begin{array}{lll}5 & \text { Summary } & 93\end{array}$

\begin{tabular}{lll}
\hline A Hamiltonian for a bead on a rigidly rotating wire & 97
\end{tabular}

A. 1 Non-relativistic limit . . . . . . . . . . . . . . . . . . . . . . . 97

A.2 Relativistic cast . . . . . . . . . . . . . . . . . . . . . 98

B Derivation of the non-relativistic transport equation 101

$\begin{array}{ll}\text { References } & 107\end{array}$ 
Acknowledgements

List of Publications 



\section{Chapter 1}

\section{Basic properties and the physics of AGN}

Galaxies are the fundamental building blocks of the universe. In the majority of cases, galaxies exhibit a quite regular structure and, by using Hubble's classification scheme for normal galaxies, may be divided in elliptical, lenticular, spiral or barred spiral galaxies.

Since the discovery of quasars (3C273) by Maarten Schmidt in 1963 (Schmidt 1963), the class of objects called "active galaxies" has become a matter of particular astrophysical interest (cf. Blandford, Netzer \& Woltjer 1990; Duschl \& Wagner 1992). "Active galaxies", to which around 1-3\% of all galaxies may be counted among, display particular spectral properties not related to ordinary stellar processes. The variable continuum emission observed from those objects is centered in a bright, compact core of the galaxy and outshines the light of all constituent stars by up to a factor of $10^{3}$. The activity centres of such galaxies with linear extension of less than one pc are usually called "Active Galactic Nuclei" (AGN) and exhibit at least one of the following properties: a high absolute luminosity, a nonthermal and often highly variable continuum emission, broad emission lines and/or rather unusual morphological structures like jets.

\subsection{Taxonomy of AGN}

The taxonomy of AGN appears to be rather complex and sometimes a bit confusing, for historically, AGN have been often classified using different 
schemes.] The so-called Seyfert galaxies (Seyfert 1943) and the quasar group constitute the two largest subclasses of AGN. Seyfert galaxies (Sy) are radio-quiet, lower luminosity $\left(M_{v}>-23\right)$ AGN which are typically found in early-type spiral galaxies. Spectroscopically they are characterised by strong, high-ionization emission lines. Seyfert galaxies could again be divided into two subclasses (cf. Khachikian \& Weedman 1974), the type 1 and type 2 Seyfert galaxies. Seyfert 1 galaxies display two distinct set of emission lines superimposed on one another, the broad lines and the narrow lines, while the less luminous Seyfert 2 only seems to have narrow lines. If the line width is interpreted as due to doppler motion around the central object, the broad lines indicate high velocities up to $10^{4} \mathrm{~km} / \mathrm{s}$ (full width half maximum) while the narrow lines imply lower velocities $<10^{3} \mathrm{~km} / \mathrm{s}$. Today however, polarization measurements indicate that broad emission lines (e.g. of hydrogen and FeII) may be also present in Seyfert 2, the polarization being probably due to scattering. Some intermediate types (e.g. Sy 1.5, Sy 1.8, Sy 1.9) based on the appearance of the optical continuum have also been introduced (e.g. Osterbrock 1981).

Quasars, on the other hand, belong to the most luminous class of AGN $\left(M_{v}<-23\right)$, showing a bluish $(\mathrm{U}-\mathrm{B}<0)$ continuum and strong broad optical emission lines. Historically, they have been distinguished from the Seyfert galaxies by being spatially unresolved on the Palomar Sky Survey photographs, implying an angular size smaller than $\sim 7^{\prime \prime}$. Most, if not all, quasars are associated with elliptical galaxies. Following Kellermann et al. 1989, quasars could be divided into radio-quiet quasars (sometimes called QSO) and radio-loud quasars (QSR) depending on whether their ratio of specific radio $(5 \mathrm{GHz})$ to optical $(680 \mathrm{THz})$ fluxes is smaller or larger than 10 . Only $10 \%$ of all quasars are radio-loud, the majority appears to be radio-quiet but detectable through optical surveys. Radio-loud quasars have been further divided into steep-spectrum radio quasars (SSRQ) and flat-spectrum radio quasars (FSRQ), according to the value of their radio spectral index $\alpha\left(S_{\nu} \propto \nu^{-\alpha}\right)$, i.e. $\alpha \leq 0.5$ for FSRQ and $\alpha>0.5$ for SSRQ.

Additionally, radio-loud but lower luminous $\left(M_{v}>-23\right)$ AGN, which reside in elliptical galaxies, are called radio galaxies (like M 87, Cen A) . Broademission line radio galaxies (BLRG) which show both, broad (asymmetric) and narrow emission lines, and the narrow-line radio galaxies (NLRG) which display only narrow lines, compose subclasses of radio galaxies. BLRG and NLRG may be regarded as the radio-loud (elliptical) counterparts of the Seyfert 1 and Seyfert 2 galaxies.

\footnotetext{
${ }^{1}$ The following overviews may serve as an introduction, e.g. Browne \& Jackson 1992; Dermer \& Schlickeiser 1992; Netzer 1990; Padovani 1999; Peterson 1997 and Woltjer 1990.
} 
LINERs (low ionization nuclear emission line region galaxies) constitute another subclass of AGN which is similar to the Sy 2 class (i.e. no broad but only narrow emission lines) except that their low-ionization lines (e.g. [OI], $[\mathrm{NII}])$ are relatively strong. Almost half of all spiral galaxies show LINER activity.

Blazars comprise one of the most interesting subclass of radio-loud AGN, characterized by their unusual rapid variability (e.g. $\Delta m \geq 0.1 \mathrm{mag} /$ day), their strong and variable optical linear polarization $\left(P_{\mathrm{opt}} \geq 3 \%\right)$ and their flat radio spectrum and featureless broad nonthermal continuum. VLBI (Very Large Baseline Interferometry) observations have revealed that some blazars are superluminal sources showing apparent transverse velocities of components larger than the speed of light (see also below). Theoretically, blazars are radio sources thought to be viewed-on (i.e. small [viewing] angles of jet axis to the line of sight), i.e. their emission is believed to be enhanced and their time scales believed to be shortened due to Doppler boosting. Three groups of radio sources have been counted to the blazar class: the optically violent variable quasars $(O V V s)$, which are characterized by rapid and large amplitude optical continuum variations; the highly polarized quasars $(H P Q s)$ with a high percentage of optical linear polarization; and the low luminous $B L L a c$ objects which show no or only weak detectable emission lines. All blazars seem to be associated with elliptical galaxies.

Historically, extragalactic radio sources have for a long time also been divided into two classes according to their radio morphology and luminosity, i.e. in the (unresolved, flat spectra) compact radio sources and the (resolved, steep spectra) extended radio sources where the emission originates from regions (e.g. lobes) more than one $\mathrm{kpc}$ from the center of the associated galaxy. Fanaroff \& Riley (1974) have introduced two subclasses for the extended radio sources, called FR I and FR II. FR I are lower luminosity $\left(\leq 2 \cdot 10^{26} \mathrm{~W} / \mathrm{Hz}\right.$ at $178 \mathrm{MHz}$ ), edge-darkened sources which show no prominent hot spots in their outer lobes. They have only weak optical emission lines and show only weak cosmological evolution. FR II sources, on the other hand, are high luminous $\left(\geq 2 \cdot 10^{26} \mathrm{~W} / \mathrm{Hz}\right.$ at $\left.178 \mathrm{MHz}\right)$, edge-brightened sources where the radio lobes are dominated by bright hot spots, probably due to shock heating in the surrounding medium. In contrast to FR I sources, they exhibit strong optical emission lines and show strong cosmological evolution. There is now mounting evidence that BL Lacs are related to FR I and quasars/OVVs to FR II radio sources. 


\subsection{Black hole - accretion disk paradigm}

Today, it is generally believed that the central engine of an AGN consists of an (eventually spinning) supermassive black hole surrounded by a hot accretion disk, where the disk is formed when gas endowed with angular momentum is accreted onto the central object (cf. Rees 1984; Frank, King \& Raine 1995). Soon after the discovery of powerful radio sources it was argued that only high-efficient conversion of gravitational into radiant energy seems to be able to account for the observed high luminosity in those objects (e.g. Zeldovič \& Novikov 1964; Lynden-Bell 1969). For, the required high efficiency of $\eta \sim 0.1$ could not be provided by any known atomic or nuclear processes leaving gravitational potential energy of infalling material (i.e. accretion) on a massive, compact object as the most likely energy source. Several lines of arguments may be added to support the black hole paradigm (note that the following overview is by no means complete): first the production, collimation and stability of AGN jets seem to be (only) plausibly explainable by MHD scenarios via the formation of rapidly rotating magnetospheres in black hole - accretion disk systems (e.g. Camenzind 1996). Secondly, the observations of (apparent) superluminal motion of radio components (see also below) indicate relativistic fluid motion in the source (Rees 1966) which in turn implies the presence of a relativistic deep gravitational potential well (cf. Blandford 1990). Thirdly, by causality arguments, the time scale for rapid variability is related to the light travel time across the size of the source. Observations of rapid variability, occasionally on time scales $\Delta t$ of the order of one minute, as observed for example in the Sy 1 galaxy NGC 6814 (cf. Kunieda et al. 1990), imply a compact source whose size is of the order of the Schwarzschildradius for a black hole of mass $M=c^{3} \Delta t /(2 G) \sim 10^{7} M_{\odot}$. Forthly, VLBA observations of water maser emitting material in the thin gaseous disk around the Sy 2 galaxy NGC 4258 show almost perfect keplerian motion (over the distance of $0.13-0.26 \mathrm{pc}$ ) around the central object indicating the presence of a central mass of $M \simeq 3 \times 10^{7} M_{\odot}$ within the inner $0.13 \mathrm{pc}$ (Miyoshi et al. 1995). Fifthly, recent reverberation mapping[ of a sample of 17 Sy 1 galaxies and two quasars yields strong evidence for central masses up to $4 \times 10^{8} M_{\odot}$ (Wandel et al. 1999). Sixthly, kinematic studies of the radio galaxy $M 87$ using optical emission lines allow a determination of the rotation curve of the ionized gas disk to a distance as close as $\simeq 5 \mathrm{pc}$ to the dynamical center, which points to the presence of a central black hole of

\footnotetext{
${ }^{2}$ Reverberation techniques use the light travel time delayed emission-line response to a continuum variation for a determination of the size and kinematics of the emission-line region, see e.g. Peterson 1997.
} 
mass $M \simeq 3 \times 10^{9} M_{\odot}$ (Maccheto et al. 1997). Seventhly, the detection of relativistic effects (i.e. relativistic Doppler and gravitational redshift) in the $\mathrm{K} \alpha \mathrm{X}$-ray emission line from ionized iron in the disk of the AGN MCG-6-3015 may add another argument to the black hole - accretion disk paradigm (Tanaka et al. 1995).

There is also increasing observational evidence that supermassive black holes do not only reside in AGN but are also present in the center of all galaxies (e.g. Rees 1984; Kormendy \& Richstone 1995; Ho 1998; Magorrian et al. 1998; Richstone et al. 1998). The most prominent example is probably our own galaxy where near-infrared imaging reveal proper motions of stars which increase with a kepler law down to separations of less than five light days from the compact radio source Sgr $\mathrm{A}^{*}$ at the dynamic center of the Milky Way. The observational findings provide strong evidence for the presence of a black hole of mass $M \simeq 3 \times 10^{6} M_{\odot}$ (Eckart \& Genzel 1996; Genzel et al. 2000; see also Falcke et al. 1993; Melia \& Falcke 2001).

Several correlations for the (putative) black holes masses in galaxies have been established in the last years. The correlation between the black hole mass and the bulge luminosity, for example, seems to be well-represented (apart from some scatter) by the relation $M_{h}=2 \times 10^{7}\left(L_{\text {bulge }} / 5 \times 10^{9} L_{\odot}\right)^{1.2}$ (e.g. Richstone et al. 1998), whereas recently a more tight correlation between the black hole mass and the velocity dispersion $\sigma$ within the half-light radius has been established, i.e. $M_{h} \simeq 1.2 \times 10^{8} M_{\odot}\left(\sigma / 200 \mathrm{~km} \mathrm{~s}^{-1}\right)^{3.75( \pm 0.3)}$ (Gebhardt et al. 2000).

There exists a critical luminosity in the black hole paradigm, called the Eddington luminosity, if one assumes spherical accretion of material. Despite the general expectation for the accreted gas to (more likely) form an accretion disk (as a consequence of being endowed with angular momentum), the assumption of spherical accretion allows the derivation of some useful quantities, generally used in accretion theory. The Eddington luminosity $L_{\text {Edd }}$ describes the maximum possible luminosity of a source of mass $M$ and is given by the condition of a balance between the (outwards directed) radiation pressure acting on an electron-proton pair and the attractive gravitational force

$$
L_{\mathrm{Edd}}=\frac{4 \pi G M m_{p} c}{\sigma_{T}} \simeq 1.2 \times 10^{46}\left(\frac{M}{10^{8} M_{\odot}}\right) \operatorname{ergs~s}^{-1}
$$

where $m_{p}$ denotes the proton mass and $\sigma_{T}$ the Thomson cross-section, $G$ is the gravitational constant and $c$ the speed of light. The related Eddington accretion rate $\dot{M}_{\text {Edd }}$, i.e. the accretion rate required to sustain an Eddington luminosity with $\eta=0.1$ efficiency of conversion of mass into radiant energy, 
may then be defined as

$$
\dot{M}_{\mathrm{Edd}}=\frac{L_{\mathrm{Edd}}}{\eta c^{2}} \simeq 2.2\left(\frac{M}{10^{8} M_{\odot}}\right) M_{\odot} \mathrm{yr}^{-1}
$$

This definition also allows a derivation of a mass-independent Eddington time (sometimes called the salpeter time), which is the e-folding time for a black hole mass accreting at the Eddington rate, i.e.

$$
t_{\mathrm{Edd}}=\frac{M}{\dot{M}_{\mathrm{Edd}}} \simeq 4.5 \times 10^{7} \mathrm{yrs} .
$$

\subsection{Superluminal motion and flux enhance- ment in relativistic jets}

In the context of AGN, the term "superluminal motion" has been introduced in order to describe the observations of (apparent) motion of radio source components with velocities exceeding the speed of light. Superluminal motion has been observed in many blazars (see above). Recent VLBA observations of 42 gamma-loud blazars, for example, reveal apparent velocities of jet components ranging up to velocities of $\sim 40 \mathrm{c}$. The distribution of apparent velocities for the general population peaks at $11 \mathrm{c}$ and shows a long high-velocity tail of sources with motion exceeding 20 c (Marscher et al. 2000). The apparent discrepancy with special relativity theory however, could be solved by assuming relativistic (but sub-c) motion of source regions at small angles to the line of sight (Rees 1966; Blandford, McKee $\&$ Rees 1977). For, consider a source moving at an inclination $i$ to the line

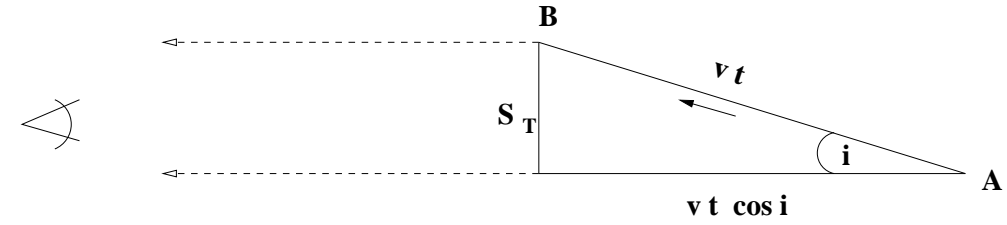

Figure 1.1: Sketch explaining the superluminal motion observed in radio sources.

of sight from point A to point B at velocity $\beta=v / c$ (see Fig. 1.1). The observed difference in arrival times for radiation emitted at $\mathrm{A}$ and $\mathrm{B}$ then appears to be shortened due to the source motion towards the observer and is given by $t_{\mathrm{obs}}=(1-\beta \cos i) t$, where $t$ denotes the (true) time interval for 
source motion from A to B. The observer measures a transverse dimension $S_{\mathrm{T}}$ of $S_{\mathrm{T}}=\beta c t \sin i$ and thus derives an apparent transverse velocity of

$$
\beta^{\mathrm{T}} c=\frac{S_{\mathrm{T}}}{t_{\mathrm{obs}}}=\frac{\beta c \sin i}{1-\beta \cos i},
$$

which may exceed the speed of light for small viewing angles. Eq. (1.4) has a maximum for $i_{m}=\arccos \beta$ where the apparent velocity becomes $\beta_{m}^{\mathrm{T}}=\gamma \beta$ with $\gamma=1 / \sqrt{1-\beta^{2}}$. Thus, for $\gamma \gg 1$ one finds $\beta_{m}^{\mathrm{T}} \gg 1$. The dependence of the apparent transverse velocity on the viewing angle is illustrated in Fig. 1.2. If the source region moves at a small viewing angle $i$ with rela-

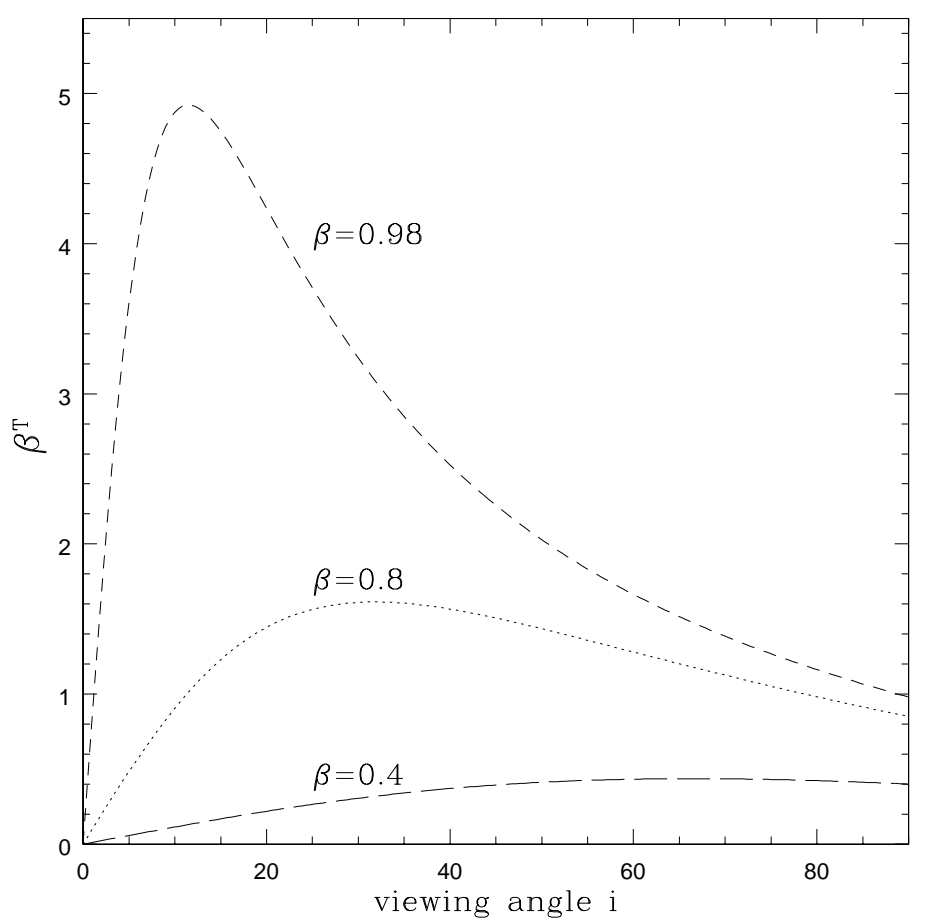

Figure 1.2: Apparent superluminal motion measured by a distant observer for different source velocities $\beta$.

tivistic speed $\beta$ towards the observer, there is another important effect, called doppler boosting or beaming, which may lead to a (drastical) enhancement of the observed flux. The Doppler boosting formulas relate the quantities measured in the comoving frame of the source to those measured in the observer frame (using Lorentz transformations). From relativistic Doppler formula 
we know, that the frequency $\nu^{\prime}$ measured in the rest frame of the source ${ }^{3}$ is related to the observed frequency $\nu$ by $\nu=\delta \nu^{\prime}$ where $\delta$ is the Doppler factor given by

$$
\delta=\frac{1}{\gamma_{b}(1-\beta \cos i)}
$$

with $\gamma_{b}$ the bulk Lorentz factor of the flow, i.e. $\gamma_{b}=1 / \sqrt{1-\beta^{2}}$. Since it can be shown that the quantity $I(\nu) / \nu^{3}$, where $I(\nu)$ denotes the intensity of radiation, is a Lorentz invariant (e.g. Rybicki \& Lightman 1979), the transformation law for the intensity becomes $I(\nu)=\delta^{3} I^{\prime}\left(\nu^{\prime}\right)$. For a resolved emission region (e.g. a blob of plasma) the flux $S(\nu)$ is transformed in the same way as the intensity. The spectral flux modulation by doppler boosting therefore can be written in the form $\mathrm{t}$

$$
S(\nu)=\delta^{3} S^{\prime}\left(\nu^{\prime}\right)=\delta^{3+\alpha} S^{\prime}(\nu)
$$

where $S^{\prime}$ is the spectral flux density measured in the comoving frame and the final equality holds if the source has a spectral index $\alpha$, i.e. $S^{\prime}\left(\nu^{\prime}\right) \propto\left(\nu^{\prime}\right)^{-\alpha}$. Doppler boosting of emission has an additional advantage of solving the problem that in several cases the deduced (variability) brightness temperature $T_{b}$ for radio (synchrotron) sources violates the inverse Compton limit of $10^{12} \mathrm{~K}$ (e.g. Wagner \& Witzel 1992). This inverse Compton limit may be derived from the requirement that the energy of (one-scattered) Compton radiation must not exceed the synchrotron radiation energy in order to avoid catastrophic losses (e.g. Kellermann \& Pauliny-Toth 1969; Melrose 1980). If Doppler boosting occurs, the observed brightness temperature appears to be enhanced by $T_{b} \sim \delta^{3} T_{b}^{\prime}$ (e.g. Blandford 1990), thus allowing an accomodation for most sources.

Beaming may also account for the $\gamma$-ray transparency of blazars (e.g. Maraschi et al. 1992). For, if beaming does not occur in blazars, the source dimensions derived from variability studies are so small, that all (co-existent) $\gamma$-ray photons, which are produced inside the source, should be absorbed through photon-photon collisions with target photons in the X-ray band, resulting in the production of $e^{+} e^{-}$pairs.

\footnotetext{
${ }^{3}$ Note, that quantities measured in the rest frame of the source are labelled with a prime superscript.

${ }^{4}$ Note, that if one additionally has to consider the redshift dependence, i.e. the expansion of the universe, the doppler factor $\delta$ in Eq. (1.6) should be replaced by $\delta /(1+z)$.
} 


\subsection{Unified models}

There have been many attempts to develop unified models which may account for the observed, wide variety of AGN-types (see sect. 1.1). Some overviews may be found in Netzer (1990), Woltjer (1990), Antonucci (1993), Urry \& Padovani (1995), Peterson (1997) and Padovani (1999). The fundamental idea behind most unification models is the assumption that the observed AGN properties depend on their viewing angle, i.e. that all AGN may intrinsically belong to the same class but are viewed under different angles. A simplified sketch of the working paradigm is shown in Fig. 1.3. According to this paradigm, an AGN consists of a black hole which is surrounded by an accretion disk. The central part is believed to be obscured by an absorbing torus with inner radius of several parsecs which is composed of dense molecular clouds. The fast moving clouds of the Broad line region (dark spots) are inside the torus, while the slower moving clouds outside the torus (grey spots) comprise the Narrow line region. Radio-loud objects additionally have a relativistic jet perpendicular to the disk, which is absent or only weak in radio-quiet objects.

Seyfert 2 galaxies are supposed to correspond to those sources which are viewed edge-on (i.e. at large viewing angle) so that the central part is obscured and can only be seen through reflected radiation due to scattering by hot electrons (black dots). Seyfert 1 galaxies, which in addition display broad emission lines, are believed to be face-on versions where the central part is unobscured. Also, BL Lacs and radio-quasars have been unified with FR I and FR II radio sources. BL Lacs are thought to correspond to the lowluminosity FR I radio sources viewed face-on, i.e. at small viewing angle, the observed properties thus being modified by Doppler boosting. Flat-spectrum (FSRQ) and steep-spectrum (SSRQ) radio quasars, on the other hand, might correspond to the more luminous FR II radio galaxies viewed at small (i.e. $<15^{\circ}$ ) and intermediate (i.e. $15^{\circ}<i<40^{\circ}$ ) angles, respectively. A simple unification scheme is shown in Tab. 1.1 using two parameters, i.e. their radio-loudness and their orientation with respect to the observer.

\subsection{The formation and collimation of jets}

Many efforts have been undertaken in order to account for the observed well-collimated, often superluminal (on parsec scales) jets in AGN. Several theoretical models for the formation and collimation of jets have been proposed. Each of these models however, seems to have its own problem (e.g. 


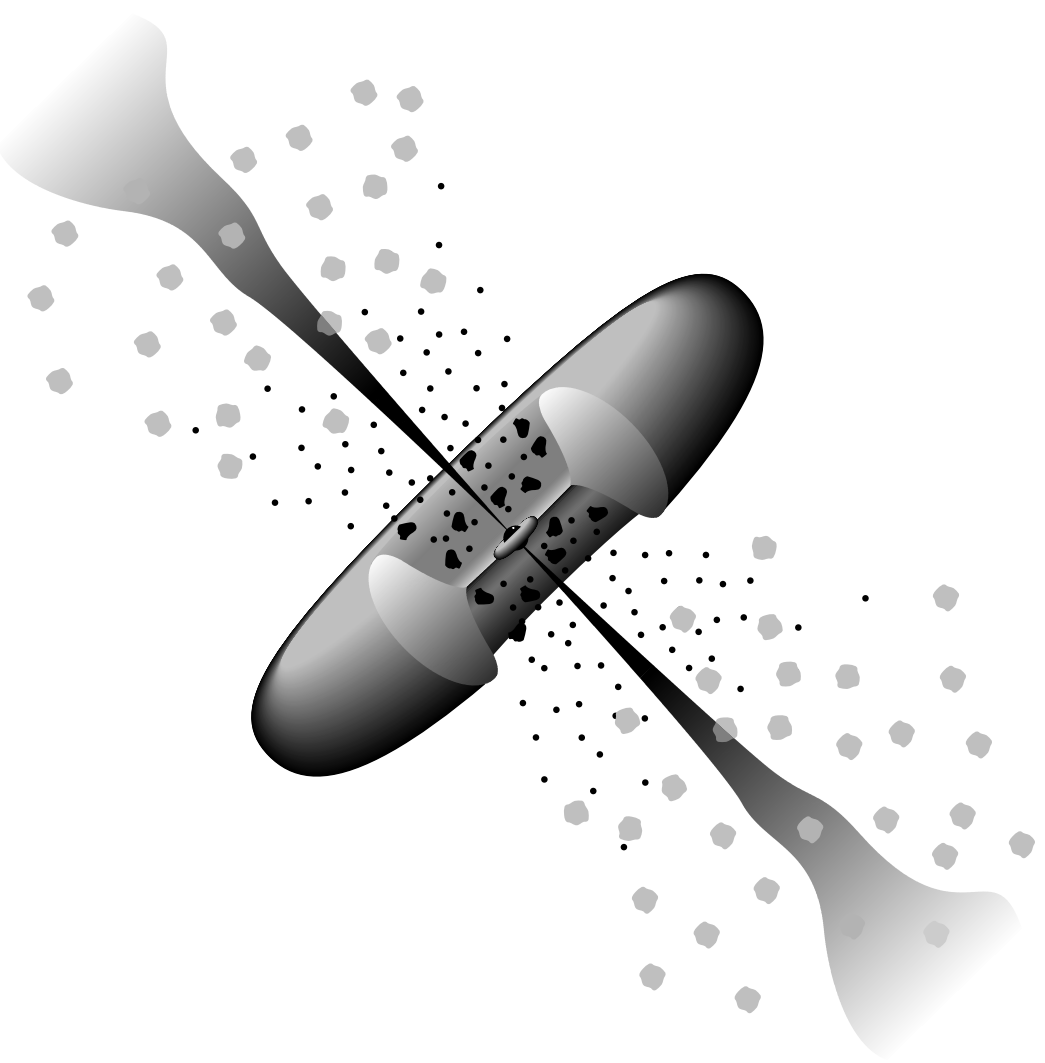

Figure 1.3: Sketch illustrating the current paradigm of (radio-loud) AGN (adopted from Urry \& Padovani 1995).

Begelman et al. 1984). Mainly three types of models may be distinguished (cf. Blandford 1990; Celotti \& Blandford 2000):

Hydrodynamical self-collimation and acceleration of an adiabatically outflowing fluid, assumed to be in pressure equilibrium with the surrounding gas, has been proposed in so-called "twin-exhausts" models (e.g. Blandford \& Rees 1974). However, for powerful radio sources the required large gas pressure would imply an X-ray luminosity exceeding the observational constraints. 


\begin{tabular}{|l|l|l|}
\hline Radio loudness & $\begin{array}{l}\text { Orientation: } \\
\text { Edge-On }\end{array}$ & $\begin{array}{l}\text { Orientation: } \\
\text { Face-On }\end{array}$ \\
\hline \hline Radio-quiet & Seyfert 2 & $\begin{array}{l}\text { Seyfert 1 } \\
\text { Radio-quiet quasars }\end{array}$ \\
\hline Radio-loud & $\begin{array}{l}\text { Fanaroff-Riley I } \\
\text { Narrow-line radio galaxies } \\
\text { Fanaroff-Riley II }\end{array}$ & $\begin{array}{l}\text { BL Lacs } \\
\text { Broad-line radio galaxies } \\
\text { Radio-quasars }\end{array}$ \\
\hline
\end{tabular}

Table 1.1: A possible, simplified AGN unification scheme, following the suggestion by Urry \& Padovani 1995 and Padovani 1999.

A second class comprises radiation pressure driven outflows assuming that the radiation pressure may be sufficient to accelerate pairs along certain directions (e.g. funnels). However, high luminosities of the order of the Eddington limit are required despite increasing observational evidence for sub-Eddington accretion in BL Lac-type objects. Additionally, due to the induced radiation drag only midly relativistic bulk velocities are expected. Today magnetohydrodynamical models seem to represent the most promising class for production and collimation of astrophysical jets (e.g. Blanford \& Znajek 1977; Blandford \& Payne 1982; Camenzind 1989; Begelman 1994). The required magnetic flux could be advected from the interstellar magnetic field via the accretion process or possibly be produced through a disc dynamo effect (e.g. Khanna \& Camenzind 1996). Co-rotation of gas with the central rotator (i.e. a rapidly spinning black hole or the surrounding accretion disk) then allows for the centrifugal acceleration. For, if the magnetic field is strong enough, (cold) matter, which is forced to corotate with the rotating field lines but free to slide along them, may be flung away from the disk by the centrifugal force. Hence the flow is accelerated centrifugally in an inner region bounded by the Alfvén surface, which in the relativistic limit is located close to the light surface (Camenzind 1986). Outside this surface however, the flow would exceed the Alfvén speed and therefore stops to be accelerated beyond this surface. The inertia of the plasma then governs the motion. Generally, the jet is assumed to be collimated by a toroidal field component which develops beyond the Alfven surface. For the associated "hoop stress" then produces a force directed towards the axis. It should be noted that there is an extensive, ongoing discussion concerning the stability of the generated solutions since (experimentally) toroidal magnetic fields are known to be unstable.

If the magnetic flux that gives rise to the jet is concentrated towards the innermost part of the disk, differential rotation of the crank could possibly be 


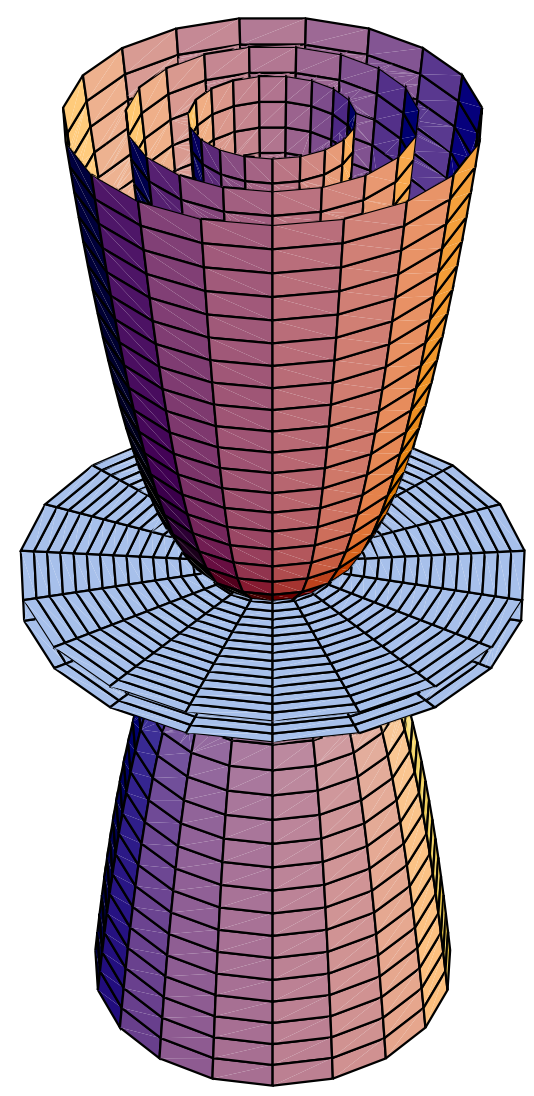

Figure 1.4: Illustration of the jet structure as expected in MHD scenarios. The jet consists of a family of nested magnetic surfaces (e.g. Camenzind \& Krockenberger 1992).

neglected and the magnetic surfaces are characterized by the same angular velocity of their footpoints (e.g. Lesch et al. 1989; Camenzind \& Krockenberger 1992). The jet may then be regarded as a family of nested magnetic surfaces rotating at constant angular velocity. Such a possible structure is illustrated in Fig. 1.4. In the high conductivity limit these surfaces can never cross and normal plasma from the disk is streaming along the surfaces. In the case where the toroidal collimation is destroyed by non-axisymmetric instabilities, powerful poloidal collimating effects are still possible (e.g. Spruit 94, Spruit et al. 1997). It is then required however, for the magnetic flux to thread the disk over several decades of radius in order to produce 
highly collimated jets. The internal velocity structure of the jet could then be still more complex (e.g. Blandford 1993).

\subsection{High energy emission and particle accel- eration in AGN}

AGN are characterized by their nonthermal emission over the whole electromagnetic spectrum from the radio to the $\gamma$-ray band, i.e. by emission over a range up to about 20 orders of magnitudes in frequency. In the case of $\gamma$-ray-loud blazars, the overall energy distribution $\left(\nu F_{\nu}\right)$ shows two broad humps, the first peaking in the infrared-optical and sometimes in the X-rays (e.g. for Mkn 421; Mkn 501; PKS 2251+158), and the second in the $\gamma$-rays (e.g. Dondi \& Ghisellini 1995). At least four BL Lac objects have recently been detected at very high energies (above $250 \mathrm{GeV}$ ) with $\gamma$-ray emission extending up to at least $10 \mathrm{TeV}$ in Mkn 421 and Mkn 501 (Catanese \& Weekes 1999).

The first hump is usually interpreted as synchrotron emission of relativistic electrons in a jet which itself moves at relativistic speed towards the observer. Synchrotron emission is produced by charged, relativistic particles in a magnetic field. Depending on the assumed intrinsic magnetic field strength, high Lorentz factors for the radiating particles are required $\left(\nu_{s} \propto \gamma^{2} B\right.$; Lorentz factor up to $\sim 10^{6}$ in leptonic models, e.g. Rieger 1997). Several acceleration mechanisms have been proposed which may explain the generation of high energy particles (e.g. via rectilinear acceleration by an electric field: Bednarek et al. 1996, Schopper et al. 1998; or via the formation of a two-stream instability: Pohl \& Schlickeiser 2000) among which Fermi-type (shock) acceleration seems to be most promising. The original idea of shock acceleration goes back to a seminal work by Fermi $(1949,1954)$ who considered the (stochastic) acceleration of particles by scattering off randomly moving magnetic mirrors (i.e. irregularities in the magnetic field), showing that a particle may gain energy $\propto(V / v)^{2}$ (sometimes called Fermi process of second order with the $V$ typical mirror velocity and $v \sim c$ the particle velocity) due to the enhanced probability for head-on collisions. In the late 1970s, an effective version of Fermi's original mechanism called "diffusive shock acceleration", was proposed (Krymsky 1977; Axford et al. 1977; Bell 1978; Blandford \& Ostriker 1978). In the underlying picture, high energy particles are supposed to pass unaffected through a shock front and scatter elastically off magnetic irregularities (Alfvén waves) in the plasma flow on either side of the shock. The acceleration mechanism then is essentially a first order process (energy 
gain $\propto V / v)$ due to the fact that in the rest frame of shock the upstream velocity $u_{1}$ exceeds the downstream velocity $u_{2}$ in which case only head-on collisions are possible. In its simplest version, this process generates a particle spectrum $N(\gamma)$ which follows a power law whose slope $s$ depends only on the shock compression ratio $\rho=u_{1} / u_{2}$ and not on the microphysics involved in the scattering process (for review, see Drury 1983; Kirk et al. 1994; Kirk \& Duffy 1999). Diffusive shock acceleration thus promises a natural explanation for the observed (apparent) universality of $\sim 2$ (i.e. $N(\gamma) \propto \gamma^{-2}$ ), predicted for strong shocks with compression ratio $\rho=4$, which is one of the main attractions of the theory. In the context of supernova remnants, the diffusive acceleration theory could reasonable account for the origin of the observed radio emission (e.g. Ball \& Kirk 1992, 1995; Duffy et al. 1995). In the case of AGN, a simple time-dependent model for the X-ray emission in blazar jets, incorporating both diffusive shock acceleration and synchrotron losses of electrons, has been recently applied by Kirk, Rieger \& Mastichiadis (1998). They successfully show that the observed spectral index variations observed in many blazars (i.e. "soft lag", "hard lag") could be understood as an interplay between the time scale for acceleration and the time scale for synchrotron losses, giving additional credit to shock acceleration mechanisms. There is an increasing consensus that the nonthermal X-ray emission in blazars might be understood as synchrotron radiation of relativistic charged particles. However, there remain considerable questions concerning the production of the observed high energy gamma-rays. Various explanations have been introduced (for review, see Schlickeiser 1996; Mannheim 1997). These explanations cover leptonic Synchrotron-Self-Compton (SSC) models, in which electrons are assumed to emit synchrotron photons directly and scatter them to high (gamma-ray) energies via the inverse-Compton process (e.g. Maraschi, Ghisellini \& Celotti 1992; Mastichiadis \& Kirk 1997). Some models suggest external photons as seed for inverse Comptom scattering (e.g. accretion disk photons: Dermer, Schlickeiser \& Mastichiadis 1992; accretion disk photons reprocessed/rescattered in the surrounding matter: Sikora, Begelman \& Rees 1994). Additionally, the importance of shock-accelerated protons have been stressed in hadronic models (e.g. Mannheim et al. 1991; Mannheim 1993). Since the radiative losses are smaller for protons than for electrons, much higher energy particles can be achieved. Pions, which are produced in proton-photon collisions, decay in pairs, neutrinos and $\gamma$-rays. The pion decay products (except for the neutrinos) will then subsequently initiate an electromagnetic cascade leading to further pairs and $\gamma$-rays until the medium becomes optically thin for the produced $\gamma$-rays. The resulting radiation could then account for the observed high energy emission.

Up to now, however, no consensus has emerged concerning the origin of the 
high energy $\gamma$-rays. For a realistic approach it seems, that to some degree all described processes might contribute to the observed high energy emission.

\subsection{The concept of this work}

The main focus of this work is concentrated on a detailed investigation of (intrinsic and extrinsic) jet rotation in AGN. Since there is increasing theoretical and observational evidence for jet rotation, we feel that there is a need for a detailed investigation which considers the impact of rotation on particle acceleration and jet morphology. By explaining the objects and related terms, the preceding comments might have set the frame for such an investigation. In the course of this work we will frequently refer to the results and expectations of the previous sections.

The present work opens with an analysis of centrifugal acceleration of test particles at the base of rigidly rotating jet magnetospheres. In magnetohydrodynamical scenarios, such rotating magnetospheres are believed to be responsible for the relativistic jet phenomena in AGN. A simple analytical approach is presented which allows of a determination of the Lorentz factor for particles following the rotating magnetic field lines. It is explicitly shown that (even if one neglects radiation losses) the energy gain of a particle is limited by the breakdown of the bead-on-the-wire approximation which occurs in the vicinity of the light cylinder.

In the subsequent chapter, the investigation of particle acceleration is extended. By using relativistic transport theory in the diffusion approximation, particle acceleration in rotating and shearing jet flows is considered. Our analysis makes recourse to previous work for the transport of cosmic rays in the galaxy by Webb (1989) and Webb, Jokipii \& Morfill (1994), but incorporates rotation profiles more relevant for AGN jets. Following an analytical approach, solutions are derived for rigidly and keplerian rotating flow profiles. In the case of rigid rotation, shearing is absent and it is shown that the energy gain might be correlated with the test particle approach of the foregoing chapter. For keplerian rotation profiles shearing is present and the solution becomes more complex. The consequences of particle acceleration by rotating and shearing jet flows and the special relevance to observations are indicated.

Occasionally, observational evidence for intrinsic jet rotation, i.e. for rotation of material inside the jet, seems not unambiguous. For, the rotation of jets due to an extrinsic cause, e.g. the precession of a jet due to the gravitational torque or the rotation of a jet in a binary system, might also mimic the ob- 
servational effects. Hence, modelling of intrinsic and extrinsic jet rotation might be of particular relevance. As an example, the extrinsic caused jet rotation in a binary system is therefore considered in a further chapter. It is shown that a binary black hole interpretation may properly account for the observed periodicity in the X-ray and $\gamma$-ray bands of the well-observed blazar Mkn 501. Additional evidence for the presence of binary black hole systems is discussed. If verified by further observations, Mkn 501 could be the first object where $\gamma$-ray astronomy contributes to the evidence for binary black hole systems. [?

\footnotetext{
${ }^{5}$ Results of chapter 2 and chapter 4 have already been published, see for example Rieger \& Mannheim (2000a,b).
} 


\section{Chapter 2}

\section{Test particle acceleration by rotating jet magnetospheres}

\subsection{Introduction}

In this chapter we consider the acceleration of test particles by rotating jet magnetospheres which are widely believed to be responsible for the relativistic jet phenomenon in active galactic nuclei (AGN). Our main focus is related to the question whether centrifugal acceleration of particles may provide an explanation for the required existence of relativistic electrons with high Lorentz factors. An answer to this question seems to be very interesting for the origin of the nonthermal, highly variable emission in AGN. Several acceleration mechanisms have been proposed which may explain the observed high energy emission extending up to $\mathrm{TeV}$ energies at least in four blazars (Mkn 421, Mkn 501, 1ES 2344+514, PKS 2155-304: for review, see Catanese \& Weekes 1999) among which Fermi-type particle acceleration mechanisms (i.e. diffusive shock acceleration) are quite promising (see sect. 1.6). However, such kind of mechanisms require a pre-accelerated seed population of electrons with Lorentz factors of the order of 100. For, in order to work efficiently, a minimum Lorentz factor $\gamma_{\min }$ of $\gamma_{\min } \sim m_{p} / m_{e}$ (where $m_{p}$ and $m_{e}$ are the proton and electron masses, respectively) seems to be demanded in the case of diffusive shock wave acceleration and resonant acceleration by magnetohydrodynamical turbulence (e.g. Lesch \& Birk 1997). This threshold condition is associated with the requirement for an electron to resonate with Alfven waves, which in turn requires $\gamma>\Omega_{e} v_{A} /(\omega c)$, where $v_{A}$ denotes the Alfven velocity and $\Omega_{e}$ is electron cyclotron frequency. Since Alfven modes only exist for $\omega \leq \Omega_{p}$, where $\Omega_{p}=e B /\left(m_{p} c\right)$ is the ion cyclotron frequency, 
one may arrive at the quoted limit. To overcome this difficulty (also referred to as the "injection problem"), it has been suggested that acceleration by whistler modes (which exists in the range $\Omega_{p}<\omega<\Omega_{e}$ ) may reduce the lower bound on the electron Lorentzfactor down to $\gamma_{\min }>\left(m_{p} / m_{e}\right)^{0.5}$, see for example Levinson (1992), Melrose (1994). While this seems possible (for a critic, however, see Lesch \& Birk 1997), there again remains the problem to be solved of how this pre-acceleration is achieved. Thus, it might be well worth considering whether in the case of AGN centrifugal acceleration at the base of a rotating jet magnetosphere may serve as an efficient acceleration mechanism, potentially providing the required pre-accelerated seed particles.

\subsection{On centrifugal acceleration in AGN}

Centrifugal acceleration models have a long and prominent history: Since the pioneer work of Gold in the late 1960s (Gold 1968, 1969), centrifugal acceleration has often been discussed in the context of pulsar emission theory (for recent contributions, see e.g. Machabeli \& Rogava 1994; Chedia et al. 1996; Gangadhara 1996; Contopoulos et al. 1999, Beskin et al. 2000). In the application to accreting black hole systems, i.e. AGN, Blandford and Payne (1982) have first pointed out that centrifugal driven outflows (jets) from magnetized accretion disks are possible, if the poloidal magnetic field direction is inclined at an angle less than $60^{\circ}$ to the radial direction (cf. sect. 1.5). However, as has been shown recently, for a very rapidly rotating black hole system this critical angle could be as large as $90^{\circ}$ (Cao 1997). In all these models, a rotating magnetosphere could emerge from the accretion disk (or the rotating black hole itself: Blandford \& Znajek 1977), initiating a plasma outflow with initially spherical shape until the flow is collimated on a scale of less than a few hundred Schwarzschild radii into an essentially cylindrical, relativistic jet (e.g. Camenzind 1995, 1996; Fendt 1997a). For illustration, a typical model topology is given in the figure below (Fig. 2.1).

Detailed calculations in such magnetohydrodynamical (MHD) scenarios for the origin of relativistic jets show, that in the MHD case centrifugal acceleration is rather limited, leading to typical maximum bulk Lorentz factors for the outflowing plasma of the order of 10 (Camenzind 1989). Despite such results however, it seems quite interesting to ask whether supra-thermal test particles (e.g. from magnetic flares on the accretion disk) could be accelerated to even higher energies at the base of such rotating jet magnetospheres. Following this question, Gangadhara \& Lesch (1997) have recently proposed a model for spinning active galactic nuclei, in which charged test particles are 

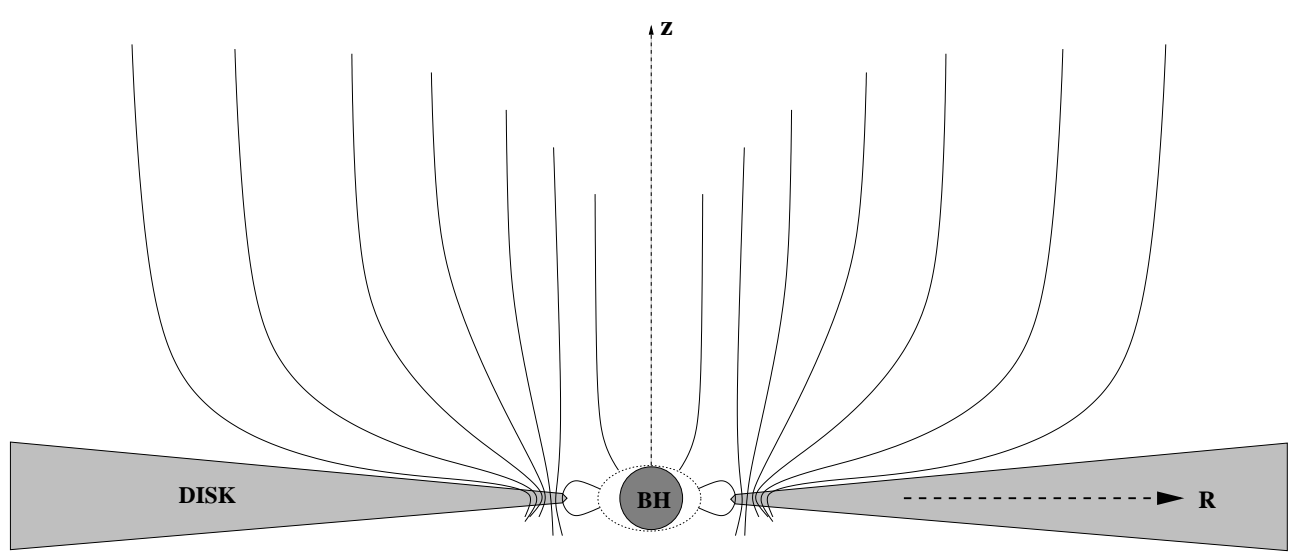

Figure 2.1: Model topology for the jet structure around a rotating black hole as expected in magnetohydrodynamical scenarios.

accelerated to very high energies by the centrifugal force while moving along rotating magnetic field lines. According to their calculations, the nonthermal $\mathrm{X}$-ray and even $\gamma$-ray emission in AGN could arise via the inverse-Compton scattering of UV-photons by centrifugal accelerated electrons.

In the following section, we reinvestigate the acceleration of charged test particles in an idealized two-dimensional model topology where the magnetic field is supposed to rotate rigidly with a fraction of the rotational velocity of the black hole (cf. Fendt 1997a). Centrifugal acceleration then occurs as a consequence of the bead-on-the-wire motion. A charged particle gains rotational energy as long as it is directed outwards. But as shown below, its energy gain is substantially limited not only by inverse-Compton losses, but also in consequence of the relativistic Coriolis force. First of all, based on an analysis of forces, the special relativistic equation of motion is derived and solved in closed form. An estimate is then given for the maximum Lorentz factor attainable in the case of AGN. At the end of this chapter, the results are discussed in the context of the particle acceleration problem for rotating AGN jets.

\subsection{Analysis of forces in a rotating reference frame}

Usually, the motion of a particle along rotating magnetic field lines is treated in the bead-on-the-wire approximation where a bead is assumed to follow the 
rotating field line and experiences centrifugal acceleration (or deceleration) while moving in the outward direction (e.g. Machabeli \& Rogava 1994; Chedia et al. 1996; Cao 1997). This simple approach yields indeed quite useful results, though - as we will show further below - such an approximation breaks down in the region near the light cylinder.

Let us first consider the forces acting on a particle in a rotating frame of reference (Gangadhara 1996; Gangadhara \& Lesch 1997). A particle with rest mass $m_{0}$ and charge $q$, which is injected at time $t_{0}$ and position $r_{0}$ with initial velocity $v_{0}$ parallel to the magnetic field line $B_{\mathrm{r}}\left(t_{0}\right)$, experiences a centrifugal force in the radial direction given by

$$
\vec{F}_{\text {cf }}=m_{0} \gamma(\vec{\Omega} \times \vec{r}) \times \vec{\Omega}
$$

where $\gamma$ is the Lorentz factor of the particle and $\vec{\Omega}=\Omega \vec{e}_{z}$ is the angular velocity of the field. Additionally, there is also a relativistic Coriolis force in the noninertial frame governed by the equation

$$
\vec{F}_{\text {cor }}=m_{0}\left(2 \gamma \frac{\mathrm{d} r}{\mathrm{~d} t}+r \frac{\mathrm{d} \gamma}{\mathrm{d} t}\right)\left(\vec{e}_{\mathrm{r}} \times \vec{\Omega}\right)
$$

which acts as a deviation-force in the azimuthal direction. In the inertial rest frame now, the particle sees the field line bending off from its initial injection position. Thus, it experiences a Lorentz force, which may be written as

$$
\vec{F}_{\mathrm{L}}=q\left(\vec{v}_{\text {rel }} \times \vec{B}\right),
$$

where $v_{\text {rel }}$ is the relative velocity between the particle and the magnetic field line and where the convention $c=1$ has been used. Due to the Lorentz force a charged particle tries to gyrate around the magnetic field line. Initially, the direction of the Lorentz force is perpendicular to the direction of the Coriolis force. But as a particle gyrates, it changes direction and eventually becomes antiparallel to the Coriolis force. Hence one expects the bead-on-the-wire approximation to hold, if the Lorentz force is not balanced by the Coriolis force. In this case the accelerated motion of the particle's guiding center due to the centrifugal force is given by

$$
\gamma \frac{\mathrm{d}^{2} r}{\mathrm{~d} t^{2}}+\frac{\mathrm{d} r}{\mathrm{~d} t} \frac{\mathrm{d} \gamma}{\mathrm{d} t}=\gamma \Omega^{2} r
$$

with $\gamma$ the Lorentz factor

$$
\gamma=\frac{1}{\sqrt{1-\Omega^{2} r^{2}-\dot{r}^{2}}}
$$


and $r$ the radial coordinate. The left hand side of Eq. (2.4) is simply given by the time-derivative of the momentum $\gamma m_{0} \mathrm{~d} r / \mathrm{d} t$. On the other hand, the bead-on-the-wire motion for the guiding center is supposed to break down, if the Coriolis force exceeds the Lorentz force, i.e. if the following inequality, given by the azimuthal components of the forces, holds:

$$
\frac{\mathrm{d} \gamma}{\mathrm{d} t}>\frac{1}{r}\left(\frac{B q v_{\mathrm{rel}}}{m_{0} \Omega}-2 \gamma \frac{\mathrm{d} r}{\mathrm{~d} t}\right) .
$$

\subsection{Analytic solution for the radial accelera- tion}

The general solution of Eq. (2.4) can be found using the simple argument that the Hamiltonian $H$ of the particle is a constant of motion (see appendix A). The Hamiltonian may be identified with the energy of the particle in the rotating reference frame. In the non-relativistic limit we know, that if $E_{0}$ denotes the energy of the particle in the inertial rest frame, then the energy $E$ in the uniformly rotating frame (angular velocity $\Omega$ ) is given by (A.4): $E=E_{0}-m_{0} \Omega^{2} r^{2}$ (e.g. Landau \& Lifshitz 1960). The generalization of this equation to the relativistic case is rather straightforward and leads to the equation [see appendix, Eq. (A.9)]

$$
H=\gamma m_{0}\left(1-\Omega^{2} r^{2}\right)=\text { const. }
$$

where $\gamma$ is the Lorentz factor defined above, see Eq. (2.5).

Assume now the general case of a particle to be injected at time $t=t_{0}$ and position $r=r_{0}$ with initial velocity $v=v_{0}$. Using Eq. (2.7) the timederivative of the radial coordinate $r$ may be written as

$$
\frac{\mathrm{d} r(t)}{\mathrm{d} t}=\sqrt{\left(1-\Omega^{2} r^{2}\right)\left[1-\tilde{m}\left(1-\Omega^{2} r^{2}\right)\right]}
$$

where

$$
\tilde{m}=\frac{1-\Omega^{2} r_{0}^{2}-v_{0}^{2}}{\left(1-\Omega^{2} r_{0}^{2}\right)^{2}} .
$$

In the particular case $r_{0}=0$, this expression reduces to the equation given in Henriksen \& Rayburn (1971).

Following Machabeli \& Rogava (1994), Eq. (2.8) could be solved analytically by introducing the variables

$$
\begin{aligned}
\kappa & =\arccos (\Omega r) \\
\lambda & =\Omega t
\end{aligned}
$$


noting that

$$
\frac{\mathrm{d} \kappa}{\mathrm{d} \lambda}=-\frac{1}{\sqrt{1-\Omega^{2} r^{2}}} \frac{\mathrm{d} r}{\mathrm{~d} t} .
$$

Thus, using Eq. (2.8) and Eq. (2.12) one has

$$
\frac{\mathrm{d} \lambda}{\mathrm{d} \kappa}=-\frac{1}{\sqrt{1-\tilde{m} \sin ^{2} \kappa}},
$$

which may be integrated yielding the formal solution

$$
\lambda=-\int_{\varphi_{0}}^{\varphi} \frac{\mathrm{d} \kappa^{\prime}}{\sqrt{1-\tilde{m} \sin ^{2} \kappa^{\prime}}}=\lambda_{0}-\int_{0}^{\varphi} \frac{\mathrm{d} \kappa^{\prime}}{\sqrt{1-\tilde{m} \sin ^{2} \kappa^{\prime}}},
$$

where $\varphi_{0}=\kappa\left(r_{0}\right)=\arccos \left(\Omega r_{0}\right)$, and where for $0<\tilde{m}<1$ the abbreviation $\lambda_{0}$ denotes a Legendre elliptic integral of the first kind

$$
\lambda_{0}=\int_{0}^{\varphi_{0}} \frac{\mathrm{d} \kappa^{\prime}}{\sqrt{1-\tilde{m} \sin ^{2} \kappa^{\prime}}} .
$$

Since $\lambda$ in Eq. (2.13) is a monotonic decreasing function of $\kappa$ for $0<\tilde{m}<1$, i.e. $\mathrm{d} \lambda / \mathrm{d} \kappa<0$, the inverse function for $\left(\lambda_{0}-\lambda\right)$, called the amplitude $\varphi=$ $\operatorname{am}\left(\lambda_{0}-\lambda\right)$, exists. By using the definition for the standard Jacobian elliptic function (e.g. Abramowitz \& Stegun 1965, p. 569ff; cf. also Tölke 1967) one finds

$$
\cos \varphi=\cos \left(\operatorname{am}\left(\lambda-\lambda_{0}\right)\right)=\operatorname{cn}\left(\lambda-\lambda_{0}\right),
$$

with cn the Jacobian elliptic cosine. Thus, by noting that $\varphi=\arccos (\Omega r)$, we finally obtain

$$
r(t)=\frac{1}{\Omega} \operatorname{cn}\left(\lambda_{0}-\Omega \mathrm{t}\right) .
$$

From Eq. (2.17) it follows that the time-derivative of $r$ could also be expressed as $\dot{r}=\operatorname{dn}\left(\lambda_{0}-\Omega t\right) \operatorname{sn}\left(\lambda_{0}-\Omega t\right)$. Note, that the Jacobian elliptic functions sn and $\mathrm{dn}$ satisfy the identities $\mathrm{sn}^{2}+\mathrm{cn}^{2}=1$ and $\tilde{m} \mathrm{sn}^{2}+\mathrm{dn}^{2}=1$.

Using Eq. (2.8), the Lorentz factor may be written as a function of the radial coordinate $r$

$$
\gamma(r(t))=\frac{1}{\sqrt{\tilde{m}}\left(1-\Omega^{2} r(t)^{2}\right)},
$$

or, on the other hand, in terms of Jacobian elliptic functions

$$
\gamma(t)=\frac{1}{\sqrt{\tilde{m}}\left[\operatorname{sn}\left(\lambda_{0}-\Omega t\right)\right]^{2}} .
$$


For the particular conditions where the injection of a test particle is described by $r\left(t_{0}=0\right)=0$ and $v\left(t_{0}=0\right)=v_{0}$, the time-dependence of the radial coordinate is given by a much simpler expression. For in this situation, $\lambda_{0}$ reduces to a complete elliptic integral $K$ of the first kind and thus, by noting that for the change of argument we have (e.g. Abramowitz \& Stegun 1965, 16.8.)

$$
\operatorname{cn}(\lambda-K)=\sqrt{1-\tilde{m}} \frac{\operatorname{sn}(\lambda)}{\operatorname{dn}(\lambda)},
$$

the time-dependence of the radial coordinate becomes

$$
r(t)=\frac{v_{0} \operatorname{sn}(\Omega \mathrm{t})}{\Omega \operatorname{dn}(\Omega \mathrm{t})} .
$$

For non-relativistic motions, where $\tilde{m} \simeq 1$, and for the special condition $r_{0}=0$, the limiting cases are given by $\operatorname{sn}(\Omega t) \rightarrow \tanh (\Omega t)$ and $\operatorname{dn}(\Omega t) \rightarrow$ $\operatorname{sech}(\Omega t)$ (cf. Abramowitz \& Stegun 1965), therefore Eq. (2.21) reduces to

$$
r(t)=\frac{v_{0}}{\Omega} \frac{\tanh (\Omega t)}{\operatorname{sech}(\Omega t)}=\frac{v_{0}}{\Omega} \sinh (\Omega t) .
$$

This expression is known to be the general solution of the equation

$$
\ddot{r}-\Omega^{2} r=0,
$$

which describes the motion of a particle due to the centrifugal force in the non-relativistic limit. In Fig. 2.2, we compute the time-dependence of the radial coordinate $r$ for different initial conditions under the (unphysical) assumption that the bead-on-the-wire motion continues until the light cylinder (with radius $r_{\mathrm{L}}$ ) is reached. For visualization, a comparison with the nonrelativistic limit is shown in Fig. 2.3. Note that in the relativistic case all particles would turn back at the light cylinder due to the reversal of the centrifugal acceleration (Machabeli \& Rogava 1994). Using the definition of the Lorentz factor, the equation for the accelerated motion, Eq. (2.4), may also be written as

$$
\frac{\mathrm{d}^{2} r}{\mathrm{~d} t^{2}}=\frac{\Omega^{2} r}{1-\Omega^{2} r^{2}}\left[1-\Omega^{2} r^{2}-2\left(\frac{\mathrm{d} r}{\mathrm{~d} t}\right)^{2}\right]
$$

(cf. Chedia et al. 1996; Kahniashvili et al. 1997) or, if entirely written as a function of the radial coordinate $r$, as

$$
\frac{\mathrm{d}^{2} r}{\mathrm{~d} t^{2}}=\Omega^{2} r\left[2 \tilde{m}\left(1-\Omega^{2} r^{2}\right)-1\right] .
$$




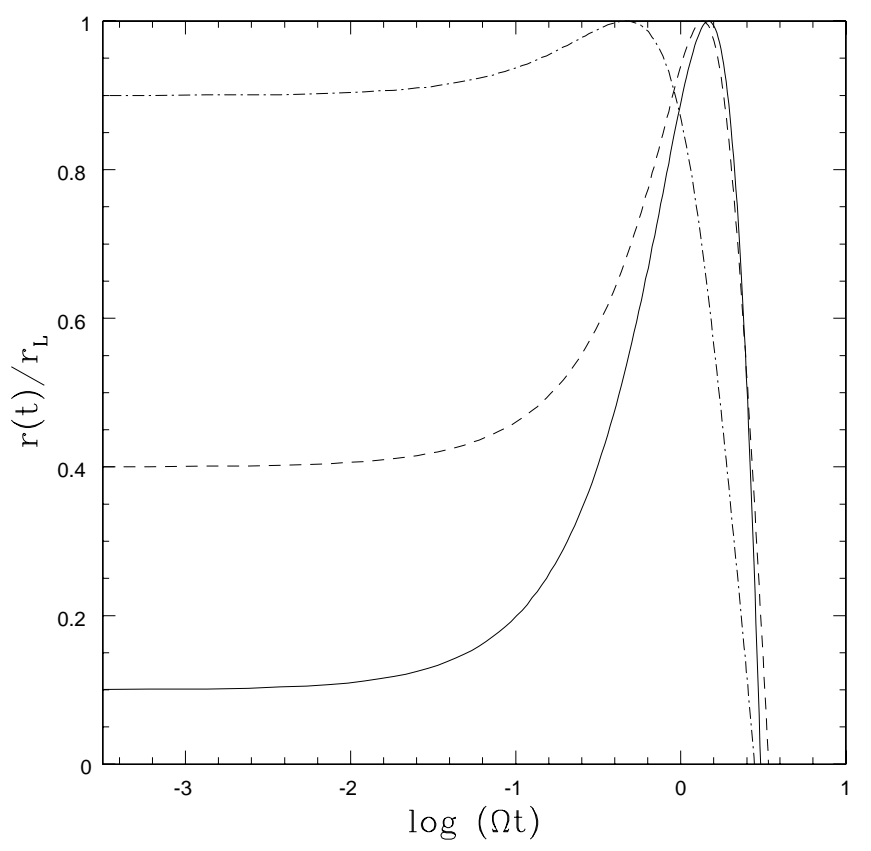

Figure 2.2: The time-dependence of the radial coordinate $r$ for bead-onthe-wire motion in the relativistic case, plotted using the initial conditions $v_{0}=0.99 c$ and $r_{0}=0.1 r_{\mathrm{L}}$ (solid line), $v_{0}=0.6 c$ and $r_{0}=0.4 r_{\mathrm{L}}$ (short dashed) and $v_{0}=0.4 c$ and $r_{0}=0.9 r_{\mathrm{L}}$ (dotted - short dashed).

By inserting the above relations, the solution for the radial acceleration could again be expressed in terms of the Jacobian elliptic functions

$$
\ddot{r}=\Omega \cdot \operatorname{cn}\left(\lambda_{0}-\Omega \mathrm{t}\right)\left[1-2 \operatorname{dn}^{2}\left(\lambda_{0}-\Omega \mathrm{t}\right)\right] .
$$

According to our simple model, one expects a charged test particle to gain energy due to rotational motion as long as it is directed outwards. Therefore the relativistic Lorentz factor increases with distance $r$ as the particle approaches the light cylinder. This is illustrated in Fig. 2.4 where the evolution of the relativistic Lorentz factor $\gamma$ is plotted as a function of $r$ for different initial velocities $v_{0}$ and fixed $r_{0}=r_{\mathrm{L}} / 10$ (using a typical light cylinder radius of $\left.r_{\mathrm{L}} \simeq 10^{15} \mathrm{~cm}\right)$. Note, that $\gamma\left(r / r_{\mathrm{L}}\right)$ is not scale-invariant with respect to the injection velocity $v_{0}$ (i.e. the injection energy). If one identifies Eq. (2.24) with the general expression for the centrifugal force, which in the non-relativistic limit reduces to the well-known classical expression, the centrifugal force changes its signs and becomes negative for $r^{2} / r_{\mathrm{L}}^{2}>1-(2 \tilde{m})^{-1}$ (see Fig. 2.5; cf. also Machabeli \& Rogava 1994). Hence, if one assumes the 


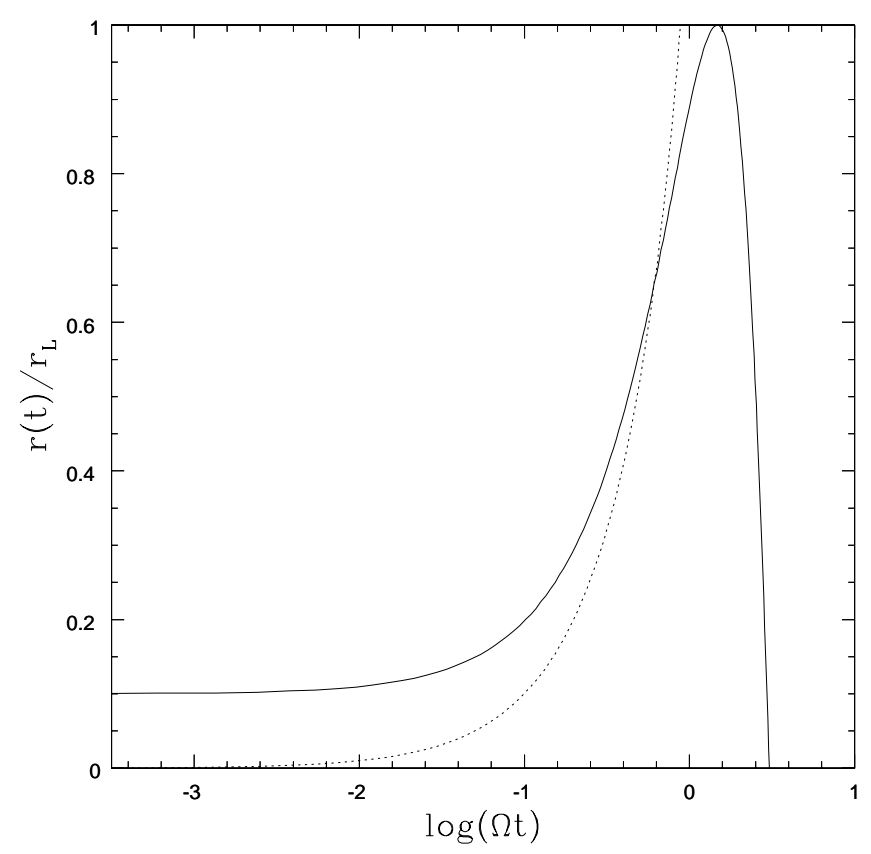

Figure 2.3: Comparision of the time-evolution of the radial coordinate $r$ in the relativistic case $\left(r_{0}=0.1 r_{\mathrm{L}}, v_{0}=0.99 \mathrm{c}\right.$, solid line) and the non-relativistic limit $r(t) \Omega / v_{0}=\sinh (\Omega \mathrm{t})$ (dotted line).

bead-on-the-wire approximation to hold in the vicinity of the light cylinder, the radial velocity becomes zero at the light cylinder and changes direction in any case. Thus $r$ will decrease (e.g. Fig. 2.2). The reversal of direction of the centrifugal force, according to which the centrifugal force may attract rotating matter towards the centre, is well-known in strong gravitational fields (for Schwarzschild geometry: Abramowicz 1990; Abramowicz \& Prasanna 1990; for Kerr geometry: Iyer \& Prasanna 1993; Sonega \& Massar 1996). For illustration, the evolution of the effective radial acceleration $a_{\mathrm{r}}=\mathrm{d}^{2} r / \mathrm{d} t^{2}$ as a function of the radial coordinate $r$, is shown in Fig. 2.5 for different initial velocities. Obviously, there exists a point where the effective acceleration, i.e. the centrifugal force, becomes negative.

\subsection{Results for the maximum Lorentz factor}

Consider now the centrifugal acceleration of electrons (rest mass $m_{0}=m_{e}$, charge $q=e$ ) via rotating magnetospheres in AGN. Imagine an electron 


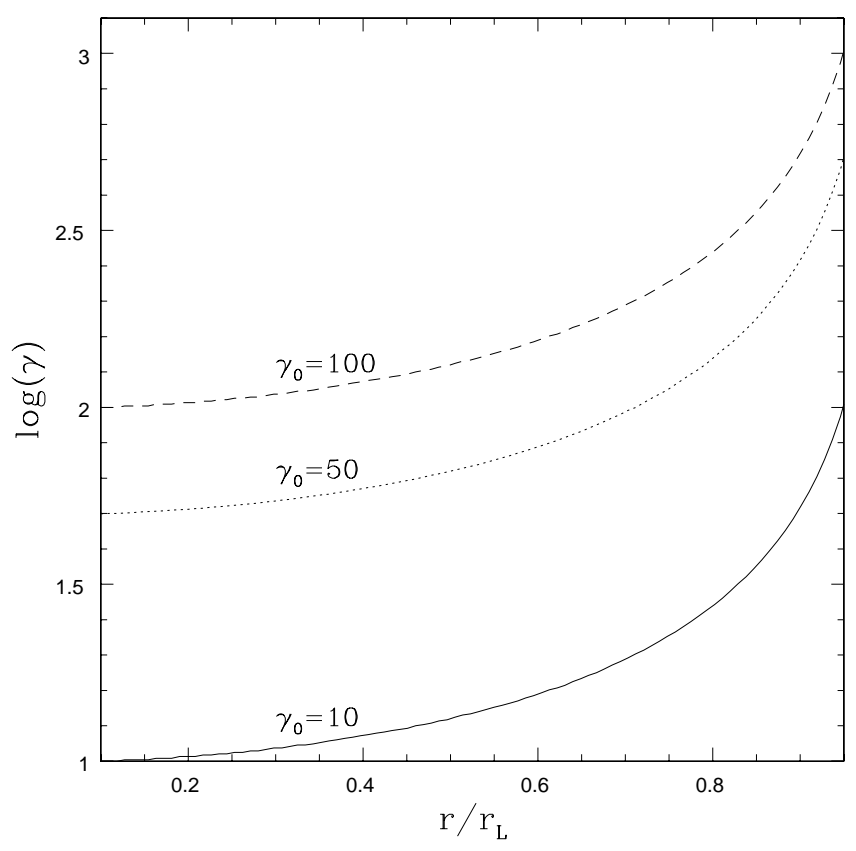

Figure 2.4: The relativistic Lorentz factor $\gamma$ for a particle approaching the light cylinder $r_{\mathrm{L}}$ using $r_{0}=r_{\mathrm{L}} / 10$ and injection Lorentz factors $\gamma_{0}=10$ (solid line), $\gamma_{0}=50$ (dotted) and $\gamma_{0}=100$ (dashed).

which moves along a rotating magnetic field line towards the light cylinder. Generally, one expects there to be at least two processes which could limit the energy gain of a particle:

First, there are inverse-Compton energy losses due to interaction with accretion disk photons: low-energy accretion disk photons are scattered to higher energies by the accelerated electrons so that the photons gain energy while the electrons lose energy. Near the disk the electrons might encounter a very strong disk radiation field, which substantially limits the maximum attainable energy (this needs not be the case if electrons are accelerated far away from the disk, e.g. Bednarek, Kirk \& Mastichiadis 1996). The maximum energy, which an electron is able to reach under the influence of inverseCompton scattering, is given at the point where the acceleration time scale equals the cooling time scale. In the case, where the energy of the photon in the electron rest frame is small compared to the energy of the electron (Thomson scattering), the cooling time scale for inverse-Compton losses can 


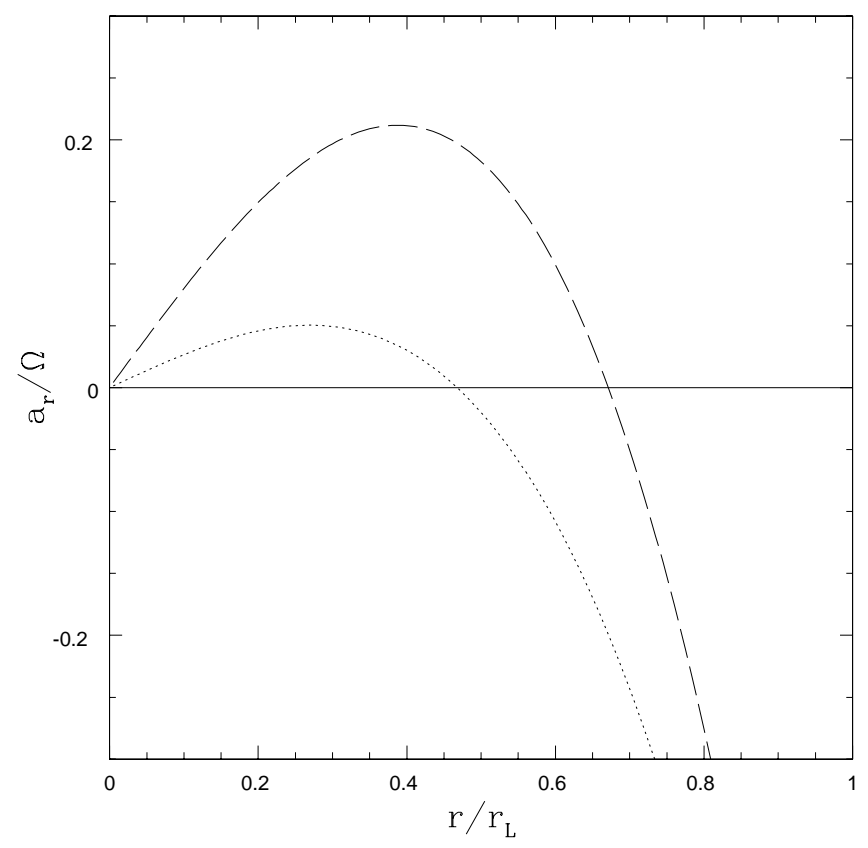

Figure 2.5: The radial acceleration $a_{\mathrm{r}}$ as a function of $r / r_{\mathrm{L}}$ for the initial conditions $r_{0}=0$ and $v_{0}=0.3 c$ (dashed), $v_{0}=0.6 \mathrm{c}$ (dotted).

be approximated by (e.g. Rybicki \& Lightman 1979)

$$
t_{\mathrm{cool}}^{\mathrm{IC}}=3 \times 10^{7} \frac{\gamma}{\left(\gamma^{2}-1\right) U_{\mathrm{rad}}}[\mathrm{s}]
$$

where $U_{\text {rad }}=\tau L_{\text {disk }} / 4 \pi r_{\mathrm{L}}^{2}$ is the energy density of the disk radiation field and $\tau \leq 1$.

Using Eq. (2.18), the acceleration time scale $t_{\text {acc }}$ may be written as:

$$
t_{\mathrm{acc}}=\gamma / \dot{\gamma}=\frac{\sqrt{1-\Omega^{2} r^{2}}}{2 \Omega^{2} r \sqrt{1-\tilde{m}\left(1-\Omega^{2} r^{2}\right)}} .
$$

By equating these two time scales, we may obtain an estimate for the maximum electron Lorentz factor $\gamma_{\max }$.

A second, general constraint, which was neither considered in the calculation by Machabeli \& Rogava (1994) nor by Gangadhara \& Lesch (1997), is given by the breakdown of the bead-on-the-wire approximation which occurs in the vicinity of the light cylinder. Beyond this point, where the Coriolis force exceeds the Lorentz force [see condition Eq. (2.6)], the particle leaves 
the magnetic field line and the rotational energy gain ceases. Hence the acceleration mechanism becomes ineffective. In the case of AGN, where the magnetic field strength is much smaller than in pulsars, this constraint may be quite important. From a mathematical point of view, the position of the breakdown of the bead-on-the-wire approximation determines the limit, up to which one may transform self-consistently into a rotating reference frame.

For illustration, we apply our calculations in the following to a typical AGN with a central black hole mass $M_{\mathrm{BH}}=10^{8} M_{\odot}$ and a light cylinder radius $r_{\mathrm{L}} \simeq 10^{15} M_{\mathrm{BH}} /\left(10^{8} M_{\odot}\right) \mathrm{cm}$, where $M_{\odot}$ denotes the solar mass. The Eddington luminosity, i.e. the maximum luminosity of a source of mass $M_{\mathrm{BH}}$ which is powered by spherical accretion [e.g. Eq. (1.1)], is given by $L_{\mathrm{Edd}} \simeq 10^{46} \mathrm{ergs}^{-1}$. Typically, we may express the disk luminosity as $L_{\text {disk }}=l_{\mathrm{e}} \times L_{\text {Edd }}$, with $10^{-4}<l_{\mathrm{e}} \leq 1$. The equipartition magnetic field strength at the radius $r$ is then given by $B(r)^{2}=2 L_{\text {disk }} / r^{2}$, where $B$ is measured in Gauss. Electrons are assumed to be injected at an initial position $r_{0} \simeq 0.4 r_{\mathrm{L}}$ with a characteristic escape velocity from the last marginally stable orbit around a black hole of $v_{0} \simeq 0.6 \mathrm{c}$. By applying the two constraints above, we may have three generic regimes for the acceleration of electrons by rotating magnetospheres:

1. the region, in which inverse-Compton losses dominate entirely over the energy gains, leading to an inefficient acceleration (generally in the case of Eddington accretion, i.e. $l_{\mathrm{e}} \sim 1$ ).

2. the region, in which inverse-Compton losses are important but not dominant (generally the sub-Eddington range: $l_{\mathrm{e}} \leq 2 \times 10^{-2}$ ). In this case the acceleration mechanism works, but there exists a maximum Lorentz factor given at the position where the energy gain is exactly balanced by losses. This is illustrated in Fig. 2.6, where we have calculated the cooling and the acceleration time scale as a function of the Lorentz factor $\gamma$ for $l_{\mathrm{e}}=5 \times 10^{-3}$. For this value, the maximum Lorentz factor is roughly $\gamma \simeq 150$. Typically, the expected maximum Lorentz factors in the range under consideration are of the order of 100 to 1000 (cf. Fig. 2.7).

3. the region, in which the inverse-Compton losses are rather unimportant (generally $l_{\mathrm{e}}<10^{-3}$ ). In this case the maximum Lorentz factor is determined by the breakdown of the bead-on-the-wire approximation [see Eq. (2.6)], which yields a general upper limit for the Lorentz factor of the order of 1000. This limit is found if one approximates $v_{\text {rel }}$ by the light velocity which amounts to the highest possible value 


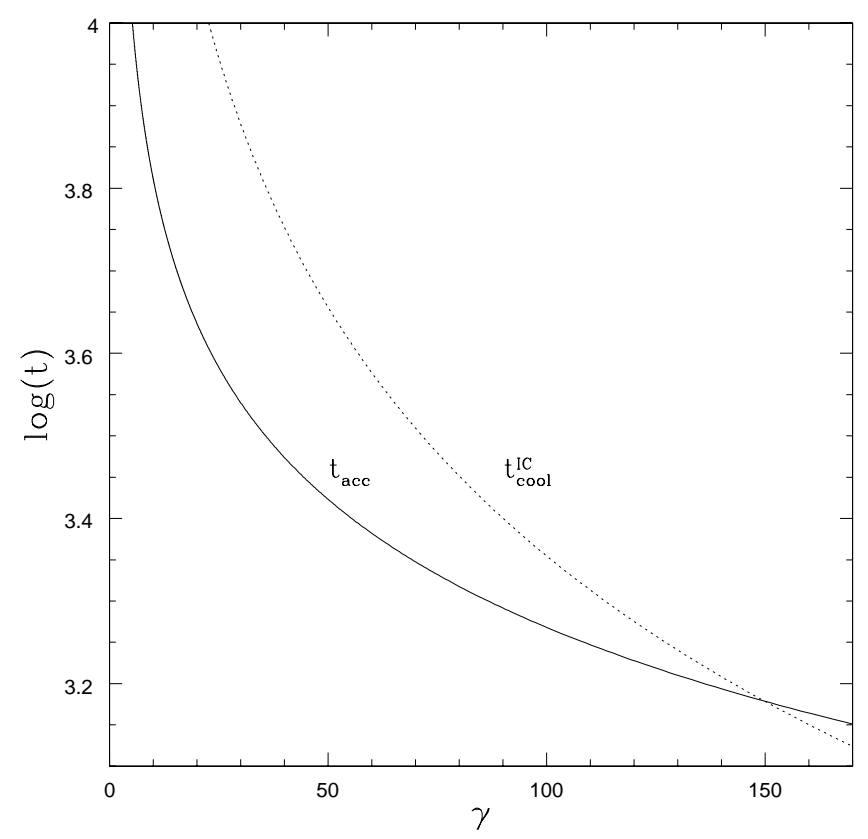

Figure 2.6: Cooling time scale $t_{\text {cool }}^{\mathrm{IC}}$ for inverse-Compton scattering, Eq. (2.27), and acceleration time scale $t_{\mathrm{acc}}$, Eq. (2.28), as a function of the Lorentz factor $\gamma$ using $l_{\mathrm{e}}=5 \times 10^{-3}$ and $\tau=1$ (i.e. $L_{\text {disk }}=5 \times 10^{43} \mathrm{ergs} / \mathrm{s}$ ). The maximum electron Lorentz factor, given at the position where the cooling time scale equals the acceleration time scale, is approximately 150.

for the Lorentz forces. The results are shown in Fig. 2.8, where we also allow the injection position to vary. It should be noted that the results, presented in Fig. 2.8, depend essentially on the assumed intrinsic magnetic field strength and the size of the light cylinder radius (i.e. the angular velocity). Generally, the breakdown condition may be written as [e.g. Eq. (2.6)]:

$$
\frac{\mathrm{d} \gamma}{\mathrm{d} t}=\frac{1}{r}\left(\frac{B e v_{\mathrm{rel}}}{m_{e} \Omega}-2 \gamma \frac{\mathrm{d} r}{\mathrm{~d} t}\right) .
$$

Using Eq. (2.18) and Eq. (2.8) the time-derivative of the Lorentz factor $\gamma$ near the light cylinder (i.e. $r \sim r_{\mathrm{L}}$ ) may be approximated by

$$
\frac{\mathrm{d} \gamma}{\mathrm{d} t} \simeq \frac{2 \Omega}{\sqrt{\tilde{m}}\left(1-\Omega^{2} r^{2}\right)^{3 / 2}},
$$

while it can be easily shown that for the range considered here, the first term in brackets on the right hand side of Eq. (2.29) is the leading 


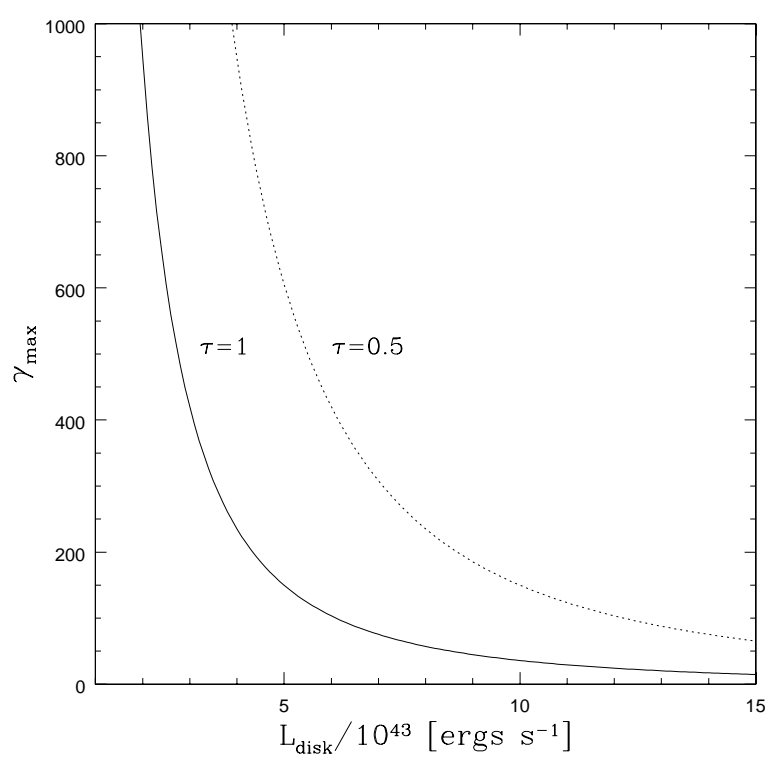

Figure 2.7: Maximum electron Lorentz factor $\gamma_{\max }$ attainable under the influence of inverse-Compton losses as a function of the disk luminosity $L_{\text {disk }}$ for $\tau=0.5$ (dotted) and $\tau=1$ (solid), where $\tau=4 \pi r_{\mathrm{L}}^{2} U_{\text {rad }} / L_{\text {disk }}$ and $U_{\text {rad }}$ is the energy density of the disk radiation field.

term (setting $v_{\text {rel }} \sim c$ ). . Hence, in a sufficiently good approximation the position of the breakdown is given by

$$
r^{2} \simeq \frac{c^{2}}{\Omega^{2}}\left[1-\left(\frac{2 m_{e} \Omega c}{B\left(r_{\mathrm{L}}\right) e \sqrt{\tilde{m}}}\right)^{2 / 3}\right]
$$

which corresponds to an upper limit for the Lorentz factor $(B$ measured in Tesla, $r_{\mathrm{L}}$ in meter)

$$
\gamma_{\max } \simeq \frac{1}{\tilde{m}^{1 / 6}}\left(\frac{B\left(r_{\mathrm{L}}\right) e}{2 m_{e} c^{2}} r_{\mathrm{L}}\right)^{2 / 3}
$$

Thus, even if one uses a magnetic field strength of $B\left(r_{\mathrm{L}}\right)=100 \mathrm{G}=$ $0.01 \mathrm{~T}$, which is roughly three times the corresponding equipartition field, the maximum Lorentz factor does not exceed $2.5 \times 10^{3}$.

\footnotetext{
${ }^{1}$ For a concrete estimate, the light velocity $c$ is explicitly written in the following expressions.
} 


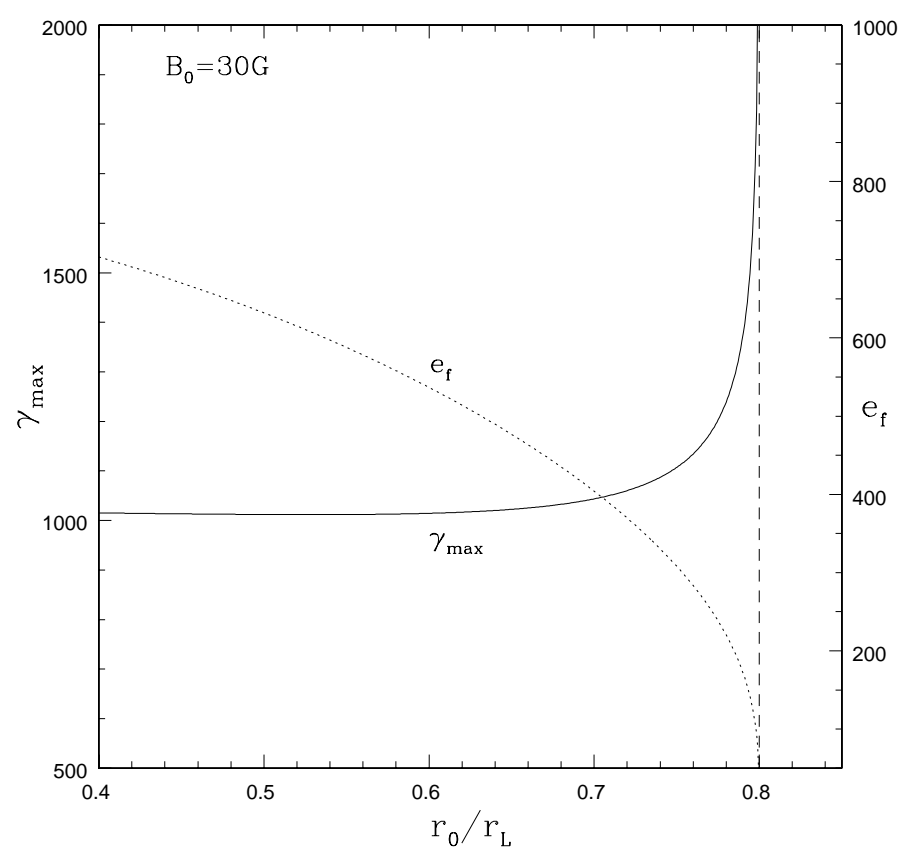

Figure 2.8: Maximum electron Lorentz factor $\gamma_{\max }$ as a function of the initial injection position $r_{0}$ for $v_{0}=0.6 \mathrm{c}$ and $B\left(r_{\mathrm{L}}\right)=30 \mathrm{G}$ (i.e. a disk luminosity $\left.L_{\text {disk }} \simeq 1.35 \times 10^{43} \mathrm{ergs} \mathrm{s}^{-1}\right)$. The dotted line shows the decrease in efficiency of energy gain $e_{f}=\gamma_{\max } / \gamma_{0}$, while the dashed line indicates the relativistic limit for injection given by the condition $1-v_{0}^{2}-\Omega^{2} r_{0}^{2}>0$.

\subsection{Discussion}

In this chapter we have considered the acceleration of charged test particles via rotating magnetospheres based on a simple model topology which is motivated by the standard MHD model for AGN (cf. Begelman 1994; Camenzind 1995; Fendt 1997a). Accordingly, the jet magnetosphere originates very closely to the central black hole from an accretion disk, with initially spherical profile until the relativistic jet is collimated to a cylindrical shape outside the light cylinder.

The centrifugal particle acceleration model described in this chapter extends the calculations by Machabeli \& Rogava (1994) and Gangadhara \& Lesch (1997). We find that the maximum Lorentz factor attainable for an electron moving along a rotating magnetic field line is substantially limited not only by radiation losses (e.g. inverse-Compton) but in particular (even if one neglects these radiation losses) by the breakdown of the bead-on-the-wire approximation which occurs in the vicinity of the light cylinder. Due to these 
limiting effects, particle acceleration by rotating jet magnetospheres seems to be rather less important for the origin of the nonthermal high energy emission in AGN. In particular, our calculations show that for sub-Eddington accreting black holes, such as black holes with advection-dominated accretion flows (e.g. Narayan \& Yi 1994; Narayan 1997), efficient pre-acceleration of electrons to Lorentz factors of the order of a few hundred might be possible, at least under the idealized conditions of our analytical model. Hence, inverse-Compton scattering of accretion disk photons seems not to provide an explanation for the high energy $\mathrm{GeV}-\mathrm{TeV}$ gamma-rays observed from AGN of the BL Lac type (cf. Kanbach 1997; Catanese 1999), which very likely accrete in a sub-Eddington mode (e.g. Celotti, Fabian \& Rees 1998).

A more quantitative estimate for the scattered photon energy may be derived by considering an optically thick standard disc around a black hole with mass $10^{8} M_{\odot}$ and light cylinder radius $r_{\mathrm{L}} \simeq 10^{15} \mathrm{~cm}$. If, as commonly assumed, the disk radiates (locally) roughly as a black body, the emission at the relevant position of the inner disc is maximized at the photon energy $\simeq 10 l_{e}^{0.25} \mathrm{eV}$ (cf. Frank, King \& Raine 1995, p. 199). Inverse-Compton scattering of accretion disc photons by centrifugally accelerated electrons with Lorentz factor $\gamma$ then yields (scattered) photons with energy of about

$$
E_{\gamma} \simeq 1.3\left(\frac{\gamma}{1000}\right)^{2}\left(10 l_{e}^{0.25}\right) \mathrm{MeV} .
$$

Using the results presented, for example, in Fig. 2.6, i.e. $l_{e}=5 \times 10^{-3}$, $\gamma_{\max }=150$, one arrives at an upper limit $E_{\gamma} \leq 80 \mathrm{keV}$. The expected photon energy is thus too low to account for the observed high energy $\mathrm{GeV}-\mathrm{TeV}$ gamma-rays in blazars.

However, it seems quite interesting to note, that the expected upper limit for the scattered photon energy falls in the range of the AGN X-ray hump between $\sim 10-100 \mathrm{keV}$. Such a hard X-ray hump is typically expected if a nonthermal power law X-ray spectrum (e.g. from a hot, nonthermal corona) is reprocessed in the cold matter in AGN (i.e. gas at amount the equivalent black body temperature, most probably in the form of an accretion disk) (cf. Guilbert \& Rees 1988; Lightman \& White 1988; George \& Fabian 1991). Photoelectric absorption then leads to a flux reduction of the X-ray spectrum below $10 \mathrm{keV}$ while Comptonization introduces a curvature above $\sim 50$ $\mathrm{keV}$. Our above calculations show that - depending on the strength of the disk radiation field - inverse-Compton scattering of accretion disk photons by centrifugally accelerated electrons may also significantly contribute to this X-ray hump.

Additionally, the acceleration of supra-thermal test particles by rotating magnetospheres might possibly provide an interesting explanation for the pre- 
acceleration which is required for efficient Fermi-type particle acceleration at larger scales in radio jets (cf. sect. 2.1).

It should be mentioned that our upper limits for the maximum Lorentz factor calculated in this chapter, essentially depend on the assumed intrinsic magnetic field and the angular frequency $\Omega$, i.e. on the size of the light cylinder radius $\left(\gamma_{\max } \propto B^{2 / 3} r_{\mathrm{L}}^{2 / 3}\right)$. Therefore it seems possible to obtain higher Lorentz factors by assuming for example, a light cylinder radius in BL Lac type objects which is much greater than $10^{15} \mathrm{~cm}$ for a black hole mass of $M_{\mathrm{BH}}=10^{8} M_{\odot}$. But in view of magnetohydrodynamic models already existing, such a possibility appears to be rather improbable (e.g. Camenzind \& Krockenberger 1992; Camenzind 1995; Fendt 1997a).

There are some restrictions on the present approach which should be kept in mind. For example, we have assumed a projected, two-dimensional geometry and rigid rotation of magnetic field lines almost up to the light cylinder and hence, concerning the last point, neglected a kind of toroidal twist (Begelman 1994), when the inertial forces overcome the tension in the field line so that the field line is swept back opposite to the sense of rotation. However, one would not expect these restrictions to alter our conclusions essentially since they should lower the upper limit for the maximum Lorentz factor by making the acceleration mechanism ineffective somewhat earlier. Another restriction is related to the use of special relativity in our analysis, which is only justified far away from the black hole. A detailed, general relativistic model may be valuable to overcome the limitations of the present approach. 


\section{Chapter 3}

\section{Particle acceleration in rotating and shearing jet flows}

\subsection{Introduction}

The observation of jets from radio-loud AGN are among the most interesting phenomena relevant to astrophysics. Today there is convincing evidence that the central engine in these AGN is a rotating, supermassive black hole surrounded by a geometrically thin accretion disk which gives rise to the formation of a pair of relativistic jets. If jets and disks are indeed symbiotic features (e.g. Falcke and Biermann, 1995), the presence of jets may however be related to a much wider class of objects.

The observations of superluminal motion and theoretical opacity arguments indicate that the plasma in these jets moves at relativistic speeds along the axis. In the case of BL Lac objects, assumed to be oriented at small viewing-angles, this may result in a strong Doppler-boosting of the observed flux (cf. sect. 1.3).

So far, theoretical acceleration and emission models usually consider rather idealized flow dynamics assuming that the relevant variable is the bulk velocity in the direction of the jet axis. In real jets however, one also expects there to be a significant velocity shear perpendicular to the jet axis. We know indeed of several observational evidence pointing to intrinsic rotation in AGN jets, e.g. in the case of NGC 4258 (Cecil et al. 1992), M87 (Biretta 1993) and for the blazar 3C345 (Schramm et al. 1993). It should be noted however, that there might remain some uncertainties as it proves not simple for any case to distinguish observationally between the intrinsic rotation of jet material and the extrinsic rotation due to jet precession or a 
compact binary system (cf. Livio 1999; Rieger \& Mannheim 2000b; see also chapt. (4).

From a theoretical point of view, intrinsic jet rotation is also expected in magnetohydrodynamical models for the formation and collimation of astrophysical jets (e.g. Begelman, 1994). In such jet models intrinsic rotation with speeds up to some fraction of the velocity of light is a natural consequence of the assumption that the flow is centrifugally accelerated from an accretion disk. It should be noted however, that the rotation profile in the jet itself does not need to be necessarily disk-like, i.e. the set of available jet rotation profiles could be much wider and might include, for example, rigid, flat and keplerian profiles (e.g. Hanasz, Sol \& Sauty 2000). In particular, rigid rotation inside a well-defined light cylinder might be related to foot points of the magnetic field lines near the last inner stable orbit (e.g. Camenzind 1996; Fendt 1997a), while more general differential rotation might be intuitively expected if there is an intrinsic connection between jet motion and the rotationg disk (cf. Blandford \& Payne 1982; Fendt 1997b; Lery \& Frank 2000; Fendt \& Memola 2001).

The acceleration of particles by shear flows has been investigated so far by several authors. A pioneer approach has been given by the kinetic analysis of Berezhko (1981; 1982a,b; 1984) and Berezhko \& Krymskii (1981, 1982). They have shown that the steady state particle distribution might follow a power law spectrum if the mean interval between scattering events increases with momentum according to a power law.

Independently, particle acceleration in the diffusion approximation at a gradual shear transition for non-relativistic flows has been analysed by Earl, Jokipii \& Morfill (1988). They derived Parker's equation, i.e. the transport equation including the well-known effects of convection, diffusion and adiabatic energy changes (Parker 1965), but augmented by new terms describing the viscous momentum transfer and the effects of inertial drifts (see also appendix B). Jokipii and Morfill (1990) have used a microscopic treatment to analyse the non-relativistic particle transport for a moving, scattering fluid which undergoes a step-function velocity change in the direction normal to the flow, showing that particles may gain energy at a rate proportional to the square of the magnitude of the velocity change. Based on a Monte Carlo simulation method, Ostrowski (1990), has studied diffusive particle acceleration at a sharp tangential velocity discontinuity involving relativistic speeds. He finds that only relativistic flows can provide conditions for efficient acceleration resulting in a very flat particle energy spectra which depends only weakly on the scattering conditions near the discontinuity (cf. also Ostrowski 1999). 
The acceleration of particles by Fermi processes in shear flows and the resultant acceleration-induced viscosity has been studied by Katz (1991). In particular, he considered the application to second-order Fermi acceleration in the low density corona due to flux tubes anchored in a keplerian accretion disk. Following this approach, Subramanian, Becker \& Kazanas (1999) have recently investigated Fermi acceleration of protons in a coupled disk-coronawind model assuming that the pressure-driven wind transforms into a jet far from the central object.

The work on (gradual) shear acceleration by Earl, Jokipii and Morfill (1988) has been extended to the relativistic regime by Webb (1989). Assuming the scattering by small-scale magnetic field irregularities to be strong enough to keep the distribution function almost isotropic in the comoving frame, i.e. the diffusion approximation to apply, he derived the relativistic diffusive particle transport equation for rotating and shearing flows employing the comoving particle momentum $p^{\prime}$. Our present approach in this chapter utilizes a simple version of the transport equation derived by Webb (cf. also Webb et al. 1994) and examines the effect of different intrinsic flow rotation profiles on the acceleration of energetic particles using a basic jet model. If intrinsic jet rotation is considered, it should be noted that particle energization may in general be a consequence of both, centrifugal and shear effects.

\subsection{Relativistic transport theory}

Using relativistic transport theory, the propagation of energetic charged particles in electromagnetic fields is governed by the relativistic Boltzmann equation (e.g. Lindquist 1966; Stewart 1971; Webb 1985)

$$
p^{\alpha} \frac{\partial f}{\partial x^{\alpha}}-\Gamma_{\beta \gamma}^{\alpha} p^{\beta} p^{\gamma} \frac{\partial f}{\partial p^{\alpha}}+q F_{\beta}^{\alpha} p^{\beta} \frac{\partial f}{\partial p^{\alpha}}=S_{c},
$$

where $f=f(\mathbf{x}, \mathbf{p})$ denotes the invariant phase space distribution function, $x^{\alpha}$ and $p^{\alpha}(\alpha=0,1,2,3)$ are the particle position and momentum four vectors, respectively, $q$ is the charge, $F_{\beta}^{\alpha}$ is the electromagnetic Faraday field tensor, $\Gamma_{\beta \gamma}^{\alpha}$ are the affine connection coefficients of the reference frame $K$ and $S_{c}$ represents the scattering of the energetic particles by MHD turbulence.

In the case of relativistic bulk flows with four velocity $u^{\alpha}$, it has been found useful to evaluate the scattering operator in the local Lorentz frame in which the fluid is at rest, i.e. in the so-called comoving frame $K^{\prime}$ (e.g. Webb 1985; 
Riffert 1988; Kirk, Schlickeiser \& Schneider 1988; cf. also appendix B).円 For in this frame $K^{\prime}$, a simple form of the scattering operator could be applied if one assumes, as in the present approach, the rest frame of the scattering centres to be essentially that of the background flow. From relativity theory we know that the metric in $K^{\prime}$ could locally be specified by the Minkowski metric $\eta_{\alpha \beta}$ with signature $(-+++)$. The quantities which are operated upon the scattering operator, e.g. the momentum, are then conveniently evaluated in this comoving frame while the time and space coordinates are measured in the laboratory frame $K$ characterized by the metric tensor $g_{\alpha \beta}$. However, since $K^{\prime}$ is in general a non-inertial coordinate system (i.e. an accelerated frame), the connection coefficients do not vanish and thus the covariant form of the Boltzmann equation is required. This covariant form may be achieved by replacing the ordinary (partial) space-time derivatives by their covariant derivatives (e.g. Webb 1989).

Starting from Eq. (3.1) and using the differential moment equations, Webb $(1989,1992)$ has derived the relativistic generalization of the particle transport equation. The scattering of high energetic particles has been modelled using a simple BKG-type time relaxation approximation (cf. Bhatnagar, Gross \& Krook 1954; also Berezhko \& Krymskii 1981; Earl, Jokipii \& Morfill 1989), i.e. using $S_{c}=-p^{\prime 0} \nu_{c}\left(f^{\prime}-f_{0}^{\prime}\right) / c$ with $\nu_{c}=1 / \tau_{c}$ the collision frequency and $f_{0}^{\prime}$ the isotropic part of the comoving frame particle distribution function $f^{\prime}$. $\tau_{c}$ denotes the mean time interval between two scattering events and may be a function of position and momentum. In the underlying physical picture, scattering of high energy particles by small-scale magnetic field irregularities carried in a collisionless plasma background flow is assumed to occur. In each scattering event the particle momentum is randomized in direction but its magnitude $p^{\prime}$ is assumed to be conserved in the (local) comoving flow frame where the electric field vanishes. Since the rest frame of the scattering centres is regarded to be essentially that of the background flow, particles would not gain energy or momentum merely by virtue of the scattering if there is no shear or rotation present (cf. also Williams \& Jokipii 1993). However if shear in the background is present, for example, the particle momentum relative to the flow changes for a particle which travels across the shear flow. Since the particle momentum in the local flow frame is preserved in the next scattering event, a net increase in particle momentum may occur (Jokipii and Morfill, 1990). Thus, if rotation and shear is present, high energy particles which do not corotate with the flow will sample the shear flow and

\footnotetext{
${ }^{1}$ In the following, quantities which are measured relative to $K^{\prime}$ are labelled with a prime superscript.
} 
may be accelerated by the centrifugal and shear effects (Webb et al, 1994).

\subsection{The general steady state transport equa- tion in the diffusion approximation}

By using a perturbation solution of the moment equations in the diffusion approximation, i.e. by assuming the deviation of the particle distribution from isotropy in the comoving frame to be small, Webb (1989) has derived a general equation describing steady state particle transport in relativistic rotating and shearing flows. From Eq.(4.4) of Webb (1989) the special relativistic diffusive particle transport equation for the mean scattering frame distribution (averaged over all momentum directions) $f_{0}^{\prime}\left(x^{\alpha}, p^{\prime}\right)=<f^{\prime}>$ may be written as

$$
\begin{array}{r}
\frac{1}{p^{\prime 2}} \frac{\partial}{\partial p^{\prime}}\left(-\frac{p^{\prime 3}}{3} c f_{0}^{\prime} \nabla_{\beta} u^{\beta}-p^{\prime}\left(p^{\prime 0}\right)^{2} \dot{u}_{\alpha} q^{\alpha}-\Gamma p^{\prime 4} \tau_{c} \frac{\partial f_{0}^{\prime}}{\partial p^{\prime}}\right) \\
+\nabla_{\alpha}\left(c u^{\alpha} f_{0}^{\prime}+q^{\alpha}\right)=0 .
\end{array}
$$

with $x^{\alpha}$ the position four vector in the laboratory frame $\mathrm{K}$, where the background plasma is in motion with four velocity $u^{\alpha}$, and $p^{\prime}$ the (magnitude of the) particle momentum as measured in the local (comoving) fluid frame $K^{\prime}$. The particle energy and momentum in this frame $K^{\prime}$ may be written as

$$
E^{\prime}=m^{\prime 2} c^{2}=p^{\prime 0} c \quad \text { and } \quad p^{\prime}=m^{\prime} v^{\prime}=\sqrt{\left(p^{\prime 0}\right)^{2}-m_{0}^{2} c^{2}},
$$

respectively, with $m^{\prime}$ the relativistic particle mass and $c$ the speed of light. The terms in the first line of Eq. (3.2) represent particle energy changes due to adiabatic expansion or compression of the flow (i.e. the term proportional to the fluid four divergence $\nabla_{\beta} u^{\beta}$ ), due to shear energization (i.e. the term involving $\Gamma$ ) and due to the fact that $K^{\prime}$ is an accelerated frame (i.e. the term $\left.\propto \dot{u}_{\alpha}\right)$. The second line gives the effects of diffusion and convection. In Eq. (3.2), $\nabla_{\alpha}$ denotes the covariant derivative while $q^{\alpha}$ denotes the heat flux. This heat flux contains a diffusive particle current plus a relativistic heat inertial term $\propto \dot{u}_{\beta}$ and is given by

$$
q^{\alpha}=-\kappa^{\alpha \beta}\left(\nabla_{\beta} f_{0}^{\prime}-\dot{u}_{\beta} \frac{\left(p^{\prime 0}\right)^{2}}{p^{\prime}} \frac{\partial f_{0}^{\prime}}{\partial p^{\prime}}\right) .
$$

As shown by Webb (1989), Eq. (3.2) could be regarded as the relativistic generalization of the non-relativistic particle transport equation first derived 
by Earl, Jokipii and Morfill (1988) (cf. appendix B).

The acceleration four vector $\dot{u}_{\alpha}$ of the comoving (or scattering) frame in Eqs. (3.2) and (3.4), is defined by

$$
\dot{u}_{\alpha}=u^{\beta} \nabla_{\beta} u_{\alpha} .
$$

The fluid energization coefficient $\Gamma$ in Eq. (3.2) represents energy changes due to viscosity. Since the acceleration of particles draws energy from the fluid flow field, one expects on the other hand the flow to be influenced by the presence of these particles. As was shown, for example, by Earl, Jokipii \& Morfill (1988) and Katz (1991), the resultant dynamical effect on the flow could be modelled by means of an (induced) viscosity coefficient. If one considers the strong scattering limit, i.e. the case where $\omega \tau_{c} \ll 1$, with $\omega$ the gyrofrequency of the particle in the scattering frame (i.e. $\left.\omega=q B^{\prime} c / p^{\prime 0}\right), \tau_{c}=$ $1 / \nu_{c}$ the mean time interval between two scattering events and $\nu_{c}$ the collision frequency, this fluid energization coefficient could be written as (Webb 1989, Eq. 34)

$$
\Gamma=\frac{c^{2}}{30} \sigma_{\alpha \beta} \sigma^{\alpha \beta}
$$

where $\sigma_{\alpha \beta}$ is the (covariant) fluid shear tensor given by

$$
\sigma_{\alpha \beta}=\nabla_{\alpha} u_{\beta}+\nabla_{\beta} u_{\alpha}+\dot{u}_{\alpha} u_{\beta}+\dot{u}_{\beta} u_{\alpha}+\frac{2}{3}\left(g_{\alpha \beta}+u_{\alpha} u_{\beta}\right) \nabla_{\delta} u^{\delta},
$$

with $g_{\alpha \beta}$ the (covariant) metric tensor. Additionally, for the strong scattering limit the spatial diffusion tensor $\kappa^{\alpha \beta}$ reduces to a simple form given by

$$
\kappa^{\alpha \beta}=\kappa\left(g^{\alpha \beta}+u^{\alpha} u^{\beta}\right), \quad \text { with } \kappa=v^{\prime 2} \tau_{c} / 3,
$$

the isotropic diffusion coefficient and $v^{\prime}$ the comoving particle speed.

\subsection{The steady state transport equation for cylindrical coordinates}

We are interested in a close investigation of particle acceleration in rotating and shearing AGN jets. Hence, for the present application, we consider an idealized (hollow) cylindrical jet model where the plasma moves along the $z$-axis at constant (relativistic) $v_{z}$ while the velocity component in the plane perpendicular to the jet axis is purely azimuthal and characterized by the angular frequency $\Omega$. For an analysis of such cylindrically rotating flows it 
proves useful to apply cylindrical coordinates $x^{\alpha}=(c t, r, \phi, z)$. We may then choose holonomic basis vectors, i.e. a coordinate basis $\left\{\mathbf{e}_{\alpha}, \alpha=0,1,2,3\right\}$ for which the commutation coefficients vanish and the affine connection coefficients reduce to the well-known Christoffel symbols. Such (non-normalized) holonomic basis vectors might be defined by $\mathbf{e}_{\alpha}=\partial \mathbf{x} / \partial x^{\alpha}$ and determine a 1 -form basis $\left\{\mathbf{e}^{\alpha}\right\}$, known as its dual basis. $x^{\alpha}$ the metric tensor $g_{\alpha \beta}$ becomes coordinate-dependent. Writing the line element $d s^{2}$ as

$$
d s^{2}=\eta_{\alpha \beta} d \xi^{\alpha} d \xi^{\beta}=g_{\alpha \beta} d x^{\alpha} d x^{\beta},
$$

with $\left(\eta_{\alpha \beta}\right)=\operatorname{diag}\{-1,1,1,1\}$ the Minkowski metric and $\xi^{\alpha}$ cartesian coordinates, and setting $\xi^{\alpha}=(c t, r \cos \phi, r \sin \phi, z)$ the covariant metric tensor may be simply written as

$$
\left(g_{\alpha \beta}\right)=\operatorname{diag}\left\{-1,1, r^{2}, 1\right\},
$$

while for the contravariant metric tensor one consequently has $\left(g^{\alpha \beta}\right)=$ $\operatorname{diag}\left\{-1,1,1 / r^{2}, 1\right\}$. Thus, except for the $g_{22}$ coefficient all partial derivatives of the metric coefficients vanish. Noting that the connection coefficients or Christoffel symbols of second order are determined by

$$
\Gamma_{k l}^{m}=\frac{g^{i m}}{2}\left(\frac{\partial g_{i k}}{\partial x^{l}}+\frac{\partial g_{l i}}{\partial x^{k}}-\frac{\partial g_{k l}}{\partial x^{i}}\right)
$$

the only non-vanishing coefficients are then given by

$$
\Gamma_{22}^{1}=-r, \quad \Gamma_{21}^{2}=\Gamma_{12}^{2}=\frac{1}{r} .
$$

Using the chosen holonomic basis the considered flow four velocity could be written in coordinate form as $u^{\alpha} \mathbf{e}_{\alpha}=\gamma \mathbf{e}_{\mathbf{0}}+(\gamma \Omega / c) \mathbf{e}_{\mathbf{2}}+\left(\gamma v_{z} / c\right) \mathbf{e}_{\mathbf{3}}$ and $u_{\alpha} \mathbf{e}^{\alpha}=-\gamma \mathbf{e}^{0}+\left(\gamma r^{2} \Omega / c\right) \mathbf{e}^{\mathbf{2}}+\left(\gamma v_{z} / c\right) \mathbf{e}^{\mathbf{3}}$, respectively, or in shortened notation as

$$
\begin{aligned}
& u^{\alpha}=\gamma\left(1,0, \Omega / c, v_{z} / c\right), \\
& u_{\alpha}=\gamma\left(-1,0, \Omega r^{2} / c, v_{z} / c\right),
\end{aligned}
$$

where the normalization

$$
\gamma=\frac{1}{\sqrt{1-\Omega^{2} r^{2} / c^{2}-v_{z}^{2} / c^{2}}}
$$

\footnotetext{
${ }^{2}$ Note that the chosen basis vectors $\mathbf{e}_{\alpha}$ are not normalized. For cylindrical coordinates the normalized basis vectors $\hat{\mathbf{e}}_{r}, \hat{\mathbf{e}}_{\phi}$ are related to the chosen ones by $\hat{\mathbf{e}}_{r}=\mathbf{e}_{1}=\mathbf{e}^{1}$ and $\hat{\mathbf{e}}_{\phi}=\mathbf{e}_{2} / r=r \mathbf{e}^{2}$.
} 
denotes the Lorentz factor of the flow and where the angular frequency may be selected to be a function of the radial coordinate, i.e. $\Omega=\Omega(r)$. We may note, that compared with the flow profile treated in the model by Webb, Jokipii \& Morfill (1994), the chosen flow velocity additionally includes a $v_{z}$-component. Also, galactic rotation (i.e. $\Omega(r) \propto 1 / r$ ) is not assumed.

For a contravariant four vector $A^{\alpha}$ the covariant derivative could generally be written as

$$
A_{\| \beta}^{\alpha}=\frac{\partial A^{\alpha}}{\partial x^{\beta}}+\Gamma_{\beta \mu}^{\alpha} A^{\mu},
$$

while for the covariant derivative of a covariant four vector $A_{\alpha}$ one has

$$
A_{\alpha \| \beta}=\frac{\partial A_{\alpha}}{\partial x^{\beta}}-\Gamma_{\alpha \beta}^{\mu} A_{\mu}
$$

Hence, for the assumed four velocity Eq. (3.13) the fluid four divergence becomes zero, i.e. $\nabla_{\beta} u^{\beta}=0$, while the fluid four acceleration Eq. (3.5) reduces to

$$
\dot{u}_{\alpha} \mathbf{e}^{\alpha}=-u^{2}\left(\Gamma_{21}^{2} u_{2}\right) \mathbf{e}^{\mathbf{1}}=-\left(\gamma^{2} \Omega^{2} r / c^{2}\right) \mathbf{e}^{\mathbf{1}} .
$$

For the components of the shear tensor Eq. (3.7) we may then derive the following relations

$$
\begin{aligned}
& \sigma_{01}=\sigma_{10}=-\left(\gamma^{3} r^{2} / c^{2}\right) \Omega \frac{d \Omega}{d r}, \\
& \sigma_{00}=\sigma_{11}=\sigma_{22}=\sigma_{33}=\sigma_{02}=\sigma_{20}=\sigma_{23}=\sigma_{32}=0, \\
& \sigma_{12}=\sigma_{21}=\left(\gamma^{3} r^{2} / c\right) \frac{d \Omega}{d r}\left(1-v_{z}^{2} / c^{2}\right), \\
& \sigma_{13}=\sigma_{31}=\left(\gamma^{3} r^{2} v_{z} / c^{3}\right) \Omega \frac{d \Omega}{d r},
\end{aligned}
$$

and thus, the viscous energization coefficient Eq. (3.6) becomes

$$
\begin{aligned}
\Gamma & =\frac{c^{2}}{30}\left(\sigma_{01} \sigma^{01}+\sigma_{10} \sigma^{10}+\sigma_{21} \sigma^{21}+\sigma_{12} \sigma^{12}+\sigma_{13} \sigma^{13}+\sigma_{31} \sigma^{31}\right) \\
& =\frac{1}{15} \gamma^{4} r^{2}\left(\frac{d \Omega}{d r}\right)^{2}\left(1-v_{z}^{2} / c^{2}\right)
\end{aligned}
$$

using that $\sigma^{01}=-\sigma_{01}, \sigma^{12}=\sigma_{12} / r^{2}$ and $\sigma^{13}=\sigma_{13}$.

For the assumed four velocity the fluid four divergence vanishes, i.e. the first term in Eq. (3.2) becomes zero, while for the second term in Eq. (3.2) we have

$$
\dot{u}_{\alpha} q^{\alpha}=\dot{u}_{1} q^{1}
$$


with the radial particle current $q^{1} \equiv q^{r} \quad[$ cf. Eq. (3.4)]

$$
q^{1} \equiv q^{r}=-\kappa\left(\frac{\partial f_{0}^{\prime}}{\partial r}+\frac{\gamma^{2} \Omega^{2} r}{c^{2}} \frac{\left(p^{0}\right)^{2}}{p^{\prime}} \frac{\partial f_{0}^{\prime}}{\partial p^{\prime}}\right)
$$

We are interested in steady state solutions of Eq. (3.2) in which case the fifth term reduces to

$$
\begin{aligned}
\nabla_{\alpha} q^{\alpha} & =\frac{\partial q^{1}}{\partial x^{1}}+\frac{\partial q^{3}}{\partial x^{3}}+\Gamma_{21}^{2} q^{1} \\
& =\frac{1}{r} \frac{\partial}{\partial r}\left(r q^{r}\right)-\kappa\left(1+\gamma^{2} v_{z}^{2} / c^{2}\right) \frac{\partial^{2} f_{0}^{\prime}}{\partial z^{2}}
\end{aligned}
$$

noting that the third component of the heat flux is given by

$$
q^{3}=-\kappa\left(1+\gamma^{2} v_{z}^{2} / c^{2}\right) \frac{\partial f_{0}^{\prime}}{\partial z}
$$

while the fourth term in Eq. (3.2) results in

$$
u^{\alpha} \nabla_{\alpha} f_{0}^{\prime}=\left(\gamma v_{z} / c\right) \frac{\partial f_{0}^{\prime}}{\partial z}
$$

Collecting the relevant terms together and introducing a source term $Q$ (which may depend on $r, z$ and $p^{\prime}$ ), the relativistic steady state transport equation for rotating and shearing flows in cylindrical coordinates could be written as

$$
\begin{array}{r}
\frac{1}{p^{\prime 2}} \frac{\partial}{\partial p^{\prime}}\left[p^{\prime}\left(p^{\prime 0}\right)^{2} \frac{\gamma^{2} \Omega^{2} r}{c^{2}} q^{r}-\frac{\gamma^{4} r^{2}}{15}\left(1-v_{z}^{2} / c^{2}\right)\left(\frac{d \Omega}{d r}\right)^{2} p^{4} \tau_{c} \frac{\partial f_{0}^{\prime}}{\partial p^{\prime}}\right] \\
+\gamma v_{z} \frac{\partial f_{0}^{\prime}}{\partial z}-\kappa\left(1+\gamma^{2} v_{z}^{2} / c^{2}\right) \frac{\partial^{2} f_{0}^{\prime}}{\partial z^{2}}+\frac{1}{r} \frac{\partial}{\partial r}\left(r q^{r}\right)=Q
\end{array}
$$

where the flow Lorentz factor is given by $\gamma=1 /\left(1-\Omega^{2} r^{2} / c^{2}-v_{z}^{2} / c^{2}\right)^{1 / 2}$.

For purely azimuthal, special relativistic flows with $v_{z}=0$, the transport equation (3.30) reduces to Eq. (5.2) derived in Webb, Jokipii \& Morfill (1994) [henceforth abbreviated as WJM 94].

As suggested by WJM 94, the derived transport equation may be cast in a more suitable form by introducing the variable

$$
\Phi=\ln (H)
$$


replacing the comoving particle momentum variable $p^{\prime}$. Following WJM 94, we may define $H$ such that $\left(\partial f_{0}^{\prime} / \partial r\right)_{H}=-q^{r} / \kappa$, with $q^{r}$ given by Eq. (3.26), the index $H$ denoting a derivative at constant $H$, i.e.

$$
H=p^{\prime 0} c \exp \left(-\int^{r} \mathrm{~d} r^{\prime} \frac{\gamma^{2} \Omega^{2} r^{\prime}}{c^{2}}\right) \text {. }
$$

For a physical interpretation of $H$ we may note, that in the case of rigid rotation (i.e. $\Omega=$ const.) $H$ could be related to the Hamiltonian for a bead on a rigidly rotating wire [cf. WJM 94; see also Eq. (3.73)].

Hence, writing $f_{0}^{\prime}\left(r, z, p^{\prime}\right) \rightarrow f_{0}^{\prime}(r, z, \Phi)$, the relevant derivatives transform like

$$
\left(\frac{\partial f_{0}^{\prime}(r, z, \Phi)}{\partial r}\right)_{\Phi}=\frac{\partial f_{0}^{\prime}\left(r, z, p^{\prime}\right)}{\partial r}+\left(\frac{\partial p^{\prime}}{\partial r}\right)_{\Phi} \frac{\partial f_{0}^{\prime}\left(r, z, p^{\prime}\right)}{\partial p^{\prime}}=-q^{r} / \kappa,
$$

using that $\partial p^{\prime} / \partial p^{0}=p^{0} / p^{\prime}$ and noting that $p^{0} c=$ $\exp \left[\int \mathrm{d} r^{\prime} \gamma^{2} \Omega^{2} r^{\prime} / c^{2}\right] \exp \Phi$. As usual, the index $\Phi$ in Eq. (3.33) denotes a derivative at constant $\Phi$. For the momentum-derivatives one finds

$$
\frac{\partial f_{0}^{\prime}\left(r, z, p^{\prime}\right)}{\partial p^{\prime}}=\frac{\partial \Phi}{\partial p^{\prime}} \frac{\partial f_{0}^{\prime}(r, z, \Phi)}{\partial \Phi}=\frac{p^{\prime}}{\left(p^{\prime 0}\right)^{2}} \frac{\partial f_{0}^{\prime}(r, z, \Phi)}{\Phi}
$$

and consequently

$$
\frac{\partial^{2} f_{0}^{\prime}\left(r, z, p^{\prime}\right)}{\partial p^{\prime 2}}=\frac{\left(p^{\prime 0}\right)^{2}-2 p^{\prime 2}}{\left(p^{\prime 0}\right)^{4}} \frac{\partial f_{0}^{\prime}(r, z, \Phi)}{\partial \Phi}+\frac{p^{\prime 2}}{\left(p^{\prime 0}\right)^{4}} \frac{\partial^{2} f_{0}^{\prime}(r, z, \Phi)}{\partial \Phi^{2}}
$$

Using Eq. (3.33) and collecting all terms together in the transport equation (3.30) which depend on $\partial f_{0}^{\prime} / \partial r$ and additionally recalling that $\kappa=$ $\left(v^{\prime 2} \tau_{c}\right) / 3$ we may arrive at

$$
\begin{array}{r}
-\kappa\left(\frac{1}{r}+\frac{\partial \kappa / \partial r}{\kappa}\right)\left(\frac{\partial f_{0}^{\prime}}{\partial r}\right)_{\Phi}+\frac{1}{p^{\prime 2}} \frac{\partial}{\partial p^{\prime}}\left[-p^{\prime}\left(p^{\prime 0}\right)^{2} \frac{\gamma^{2} \Omega^{2} r}{c^{2}} \kappa\right]\left(\frac{\partial f_{0}^{\prime}}{\partial r}\right)_{\Phi}= \\
-\kappa \frac{1}{r}(1+\beta)\left(\frac{\partial f_{0}^{\prime}}{\partial r}\right)_{\Phi}-\kappa[3+\alpha]\left(\frac{p^{\prime 0}}{p^{\prime}}\right)^{2} \frac{\gamma^{2} \Omega^{2} r}{c^{2}}\left(\frac{\partial f_{0}^{\prime}}{\partial r}\right)_{\Phi}
\end{array}
$$

where the position and momentum dependence of the collision time $\tau_{c}$ (and thus of the diffusion coefficient) has been caught into the definition of the variables $\alpha$ and $\beta$, i.e. $\alpha$ and $\beta$ are given by

$$
\begin{aligned}
& \alpha=\frac{\partial \ln \tau_{c}}{\partial \ln p^{\prime}}, \\
& \beta=\frac{\partial \ln \tau_{c}}{\partial \ln r},
\end{aligned}
$$


respectively. Eq. (3.36) has been obtained by making use of

$$
\begin{aligned}
v^{\prime 2} & =c^{2} p^{2} /\left(p^{\prime 0}\right)^{2} \quad \text { and } \\
\frac{\partial \kappa}{\partial p^{\prime}} & =\frac{1}{3}\left(\frac{\partial v^{\prime 2}}{\partial p^{\prime}} \tau_{c}+v^{\prime 2} \frac{\partial \tau_{c}}{\partial p^{\prime}}\right), \\
\frac{\partial \tau_{c} / \partial p^{\prime}}{\tau_{c}} & =\frac{1}{p^{\prime}} \alpha .
\end{aligned}
$$

In a similar manner, the terms depending on $\partial f_{0}^{\prime} / \partial p^{\prime}$ in Eq. (3.30) may be rewritten using Eq. (3.34) as

$$
\begin{array}{r}
-\frac{1}{p^{\prime 2}}\left[\frac{\partial}{\partial p^{\prime}}\left(\frac{\gamma^{4} r^{2}}{15}\left(1-v_{z}^{2} / c^{2}\right)\left(\frac{\mathrm{d} \Omega}{\mathrm{d} r}\right)^{2} p^{\prime 4} \tau_{c}\right)\right] \frac{\partial f_{0}^{\prime}\left(r, z, p^{\prime}\right)}{\partial p^{\prime}}= \\
-\kappa \frac{\gamma^{4} r^{2}}{5 c^{2}}\left(1-v_{z}^{2} / c^{2}\right)[4+\alpha]\left(\frac{\mathrm{d} \Omega}{\mathrm{d} r}\right)^{2} \frac{\partial f_{0}^{\prime}(r, z, \Phi)}{\partial \Phi}
\end{array}
$$

Similarly, for the terms in Eq. (3.30) depending on $\partial^{2} f_{0}^{\prime} / \partial p^{\prime 2}$ one finds

$$
\begin{aligned}
& \frac{1}{p^{\prime 2}}\left(\frac{\gamma^{4} r^{2}}{15 c^{2}}\left(1-\frac{v_{z}^{2}}{c^{2}}\right)\left(\frac{\mathrm{d} \Omega}{\mathrm{d} r}\right)^{2} p^{\prime 4} \tau_{c}\right) \frac{\partial^{2} f_{0}^{\prime}\left(r, z, p^{\prime}\right)}{\partial p^{\prime 2}}= \\
& \kappa \frac{\gamma^{4} r^{2}}{5 c^{2}}\left(1-\frac{v_{z}^{2}}{c^{2}}\right)\left(\frac{\mathrm{d} \Omega}{\mathrm{d} r}\right)^{2}\left(\left[1-2 \frac{p^{\prime 2}}{\left(p^{\prime 0}\right)^{2}}\right] \frac{\partial f_{0}^{\prime}(r, z, \Phi)}{\partial \Phi}+\frac{p^{\prime 2}}{\left(p^{\prime 0}\right)^{2}} \frac{\partial^{2} f_{0}^{\prime}(r, z, \Phi)}{\partial \Phi^{2}}\right)
\end{aligned}
$$

where Eq. (3.35) has been used.

Now, collecting all relevant terms together the steady state transport equation (3.30) may finally be rewritten as

$$
\begin{aligned}
& \frac{\partial^{2} f_{0}^{\prime}}{\partial r^{2}}+\left(\frac{1+\beta}{r}+[3+\alpha] \frac{\gamma^{2} \Omega^{2} r}{c^{2}}\left(\frac{p^{\prime 0}}{p^{\prime}}\right)^{2}\right) \frac{\partial f_{0}^{\prime}}{\partial r} \\
+ & \frac{\gamma^{4} r^{2}}{5 c^{2}}\left(1-v_{z}^{2} / c^{2}\right)\left(\frac{\mathrm{d} \Omega}{\mathrm{d} r}\right)^{2}\left(\left[5+\alpha-2\left(\frac{p^{\prime}}{p^{\prime 0}}\right)^{2}\right] \frac{\partial f_{0}^{\prime}}{\partial \Phi}+\left(\frac{p^{\prime}}{p^{\prime 0}}\right)^{2} \frac{\partial^{2} f_{0}^{\prime}}{\partial \Phi^{2}}\right) \\
- & \frac{\gamma v_{z}}{\kappa} \frac{\partial f_{0}^{\prime}}{\partial z}+\left(1+\gamma^{2} v_{z}^{2} / c^{2}\right) \frac{\partial^{2} f_{0}^{\prime}}{\partial z^{2}}=-\frac{Q}{\kappa}
\end{aligned}
$$

with $f_{0}^{\prime} \equiv f_{0}^{\prime}(r, z, \Phi)$ and where the $r$-derivatives should be understood as derivatives keeping $\Phi$ constant, i.e. we may treat $r, z, \Phi$ as independent variables. Again, for $v_{z}=0$ the partial differential equation (3.44) reduces to the steady state transport equation given in WJM 94 [e.g. see their 
Eq. (5.5)].

For the present purpose, we are especially interested in the case of highly relativistic particles with $p^{\prime 0} \simeq p^{\prime}$ which allows a further simplification of Eq. (3.44), yielding

$$
\begin{aligned}
\frac{\partial^{2} f_{0}^{\prime}}{\partial r^{2}} & +\left(\frac{1+\beta}{r}+[3+\alpha] \frac{\gamma^{2} \Omega^{2} r}{c^{2}}\right) \frac{\partial f_{0}^{\prime}}{\partial r} \\
& +\frac{\gamma^{4} r^{2}}{5 c^{2}}\left(1-v_{z}^{2} / c^{2}\right)\left(\frac{\mathrm{d} \Omega}{\mathrm{d} r}\right)^{2}\left([3+\alpha] \frac{\partial f_{0}^{\prime}}{\partial \Phi}+\frac{\partial^{2} f_{0}^{\prime}}{\partial \Phi^{2}}\right) \\
& -\frac{\gamma v_{z}}{\kappa} \frac{\partial f_{0}^{\prime}}{\partial z}+\left(1+\gamma^{2} v_{z}^{2} / c^{2}\right) \frac{\partial^{2} f_{0}^{\prime}}{\partial z^{2}}=-\frac{Q}{\kappa}
\end{aligned}
$$

\subsection{On Green's function solutions for the steady state transport equation}

\subsubsection{Fourier method and the Green's functions for in- finite domains}

For general applications one may search for Green's function solutions of the steady state transport equation (see also WJM 94), i.e. solutions of Eq. (3.45) with source term

$$
Q=q_{0} \delta\left(r-r_{s}\right) \delta\left(p^{\prime}-p_{s}^{\prime}\right) \delta\left(z-z_{s}\right),
$$

or equivalently (i.e. utilizing the properties of the delta function) with source term

$$
Q=\frac{q_{0}}{p_{s}^{\prime}} \delta\left(r-r_{s}\right) \delta\left(\Phi-\Phi_{s}\right) \delta\left(z-z_{s}\right)
$$

describing monoenergetic injection of particles with momentum $p^{\prime}=p_{s}^{\prime}$ at position $r=r_{s}, z=z_{s}$. For consistency, the constant $q_{0}$ in Eqs. (3.46), (3.47) has to be defined such, that the relevant expression satisfies the requirement that $\delta\left(\vec{r}-\vec{r}_{s}\right) \delta\left(\vec{p}^{\prime}-\vec{p}_{s}{ }^{\prime}\right)$ vanishes unless $r=r_{s}, \phi=\phi_{s}, p^{\prime}=p_{s}^{\prime}$ and integrate to unity (or $N_{s}$ if $N_{s}$ particles are injected) over all space and momentum directions. Using cylindrical coordinates, performing the $\phi$-integration and taking into account that $f_{0}^{\prime}$ is assumed to be independent of momentum directions (isotropic) one arrives at the following condition

$$
\begin{aligned}
& \iiint \int N_{s} \delta\left(\vec{r}-\vec{r}_{s}\right) \delta\left(\vec{p}^{\prime}-\vec{p}_{s}{ }^{\prime}\right) r \mathrm{~d} r \mathrm{~d} \phi \mathrm{d} z\left(4 \pi p^{\prime 2}\right) \mathrm{d} p^{\prime} \\
& =2 \pi\left(4 \pi p_{s}^{\prime 2} r_{s}\right) q_{0} \iiint \delta\left(r-r_{s}\right) \delta\left(p^{\prime}-p_{s}^{\prime}\right) \delta\left(z-z_{s}\right) \mathrm{d} r \mathrm{~d} p^{\prime} \mathrm{d} z=N_{\xi}
\end{aligned}
$$


which requires

$$
q_{0}=\frac{N_{s}}{8 \pi^{2} p_{s}^{\prime 2} r_{s}} .
$$

In order to solve the inhomogeneous differential equation Eq. (3.45), Fourier techniques are applied in the following, i.e. we use the double Fourier transform defined by

$$
F_{0}^{\prime}(r ; \mu, \nu)=\int_{-\infty}^{\infty} d z \int_{-\infty}^{\infty} d \Phi \exp [i(\nu \Phi+\mu z)] f_{0}^{\prime}(r, z, \Phi),
$$

where the inverse Fourier transform is given by

$$
f_{0}^{\prime}(r, z, \Phi)=\frac{1}{4 \pi^{2}} \int_{-\infty}^{\infty} d \mu \int_{-\infty}^{\infty} d \nu \exp [-i(\nu \Phi+\mu z)] F_{0}^{\prime}(r ; \mu, \nu)
$$

Denoting the Fourier transform of the source term $-Q / \kappa$ by $\tilde{Q}$, we have

$$
\begin{aligned}
\tilde{Q} & =-\int_{-\infty}^{\infty} d z \int_{-\infty}^{\infty} d \Phi \exp [i(\nu \Phi+\mu z)] \frac{q_{0}}{\kappa p_{s}^{\prime}} \delta\left(r-r_{s}\right) \delta\left(\Phi-\Phi_{s}\right) \delta\left(z-z_{s}\right) \\
& =-\frac{q_{0}}{\kappa_{s} p_{s}^{\prime}} \exp \left[i\left(\nu \Phi_{s}+\mu z_{s}\right)\right] \delta\left(r-r_{s}\right)
\end{aligned}
$$

By taking the Fourier transform of the steady state transport equation (3.45) one finds

$$
\begin{aligned}
& \frac{\partial^{2} F_{0}^{\prime}}{\partial r^{2}}+\left(\frac{1+\beta}{r}+[3+\alpha] \frac{\gamma^{2} \Omega^{2} r}{c^{2}}\right) \frac{\partial F_{0}^{\prime}}{\partial r} \\
& -\left[\frac{\gamma^{4} r^{2}\left(1-\frac{v_{z}^{2}}{c^{2}}\right)}{5 c^{2}}\left(\frac{\mathrm{d} \Omega}{\mathrm{d} r}\right)^{2}\left([3+\alpha] i \nu+\nu^{2}\right)-\frac{\gamma v_{z}}{\kappa} i \mu+\left(1+\gamma^{2} \frac{v_{z}^{2}}{c^{2}}\right) \mu^{2}\right] F_{0}^{\prime} \\
& =\tilde{Q} .
\end{aligned}
$$

Let $F_{G}^{\prime}$ be the Green's solution of this equation satisfying homogeneous, i.e. zero Dirichlet boundary conditions, then Fourier inversion [i.e. Eq. (3.51)] yields the Green's function $f_{G}^{\prime}\left(r, z, p^{\prime} ; r_{s}, z_{s}, p_{s}^{\prime}\right)$ for infinite domains.

\subsubsection{On the method of images and the Green's func- tions for bounded domains}

Fourier technique yields the Green's function for infinite domains. The proper Green's function for bounded domains, i.e. for finite $z$, may be obtained by the method of images (e.g. Morse \& Feshbach 1953, pp. 812-816) in the case 
where the boundaries are restricted to straight lines in two dimensions or planes in three dimensions. For homogeneous Dirichlet boundary conditions the method of images involves the introduction of a line charge of opposite sign at the relevant image point, the resultant potential satisfying the boundary conditions thus being regarded as a superposition of the source and its image. Consider now an idealized jet model where $\mho \mho$ denotes the appropriate volume over which the source $Q=Q\left(r, z, p^{\prime}\right)$ may be distributed and let $\mho$ be defined by the Cartesian product

$$
\mho=V \times\left[p_{1}^{\prime}, p_{2}^{\prime}\right]
$$

where $V$ marks the volume specified for an application to cylindrical jets

$$
V=\left\{(r, \phi, z): 0<r_{\text {in }}<r<r_{\text {out }}, 0 \leq \phi<2 \pi,-h_{\min }<z<h_{\max }\right\}
$$

with $r_{\text {in }}$ the jet inner radius and $r_{\text {out }}$ its relevant outer radius and $h_{\min }, h_{\max } \geq$ 0 . For simplicity one may choose momentum boundaries fixed at $p_{1}^{\prime}=0$, $p_{2}^{\prime}=\infty$. Determining the general solution of the steady state transport equation with Dirichlet boundary conditions (not necessarily homogeneous, i.e. zero Dirichlet boundary conditions) on the boundary $\partial \mho$ then involves specifying the Green's function solution satisfying homogeneous boundary conditions on $\partial \mho$, i.e.

$$
\begin{aligned}
f_{G}^{\prime}\left(r_{\mathrm{in}}, z, p^{\prime} ; r_{s}, z_{s}, p_{s}^{\prime}\right) & =f_{G}^{\prime}\left(r_{\mathrm{out}}, z, p^{\prime} ; r_{s}, z_{s}, p_{s}^{\prime}\right)=0 \text { and } \\
f_{G}^{\prime}\left(r,-h_{\min }, p^{\prime} ; r_{s}, z_{s}, p_{s}^{\prime}\right) & =f_{G}^{\prime}\left(r, h_{\max }, p^{\prime} ; r_{s}, z_{s}, p_{s}^{\prime}\right)=0 .
\end{aligned}
$$

As stated above, Fourier technique yields the Green's function solution for infinite domains (i.e. for $|z| \rightarrow \infty$ ) while the method of images may be applied to derive the Green's function for bounded domains (i.e. for $0<|z|<$ $h$ ). In order to illustrate the method of images in the case considered here, suppose that the solution for the steady state transport equation Eq. (3.53) which satisfies homogeneous Dirichlet boundary conditions, could be written in the form

$$
F_{G}^{\prime}\left(r, r_{s} ; \mu, \nu\right)=\tilde{F}_{0}^{\prime}\left(r, r_{s} ; \mu, \nu\right) \exp \left[i \mu z_{s}+i \nu \Phi_{s}\right] .
$$

Then by Fourier inversion (cf. Eq. (3.51)) the required Green's function for infinite domains is given by

$$
\begin{aligned}
f_{G}^{\prime}\left(r, z, \Phi ; r_{s}, z_{s}, \Phi_{s}\right)= & \frac{1}{4 \pi^{2}} \int_{-\infty}^{\infty} \mathrm{d} \mu \int_{-\infty}^{\infty} \mathrm{d} \nu \tilde{F}_{0}^{\prime}\left(r, r_{s} ; \mu, \nu\right) \\
& \times \exp \left[-i \mu\left(z-z_{s}\right)-i \nu\left(\Phi-\Phi_{s}\right)\right]
\end{aligned}
$$


Evaluating the $\mu$-integration may now be done by performing contour integration and taking into account the possible poles of $\tilde{F}_{0}^{\prime}$ which might be a function of $\mu$. Using the calculus of residues, the value of the contour integral then equals $2 \pi i$ times the sum of the residues at the relevant poles enclosed by the chosen contour. Thus, by choosing the proper contour (e.g. see Morse \& Feshbach 1953, p. 416) one may obtain a sum over $n$ which entails terms of the form $\exp \left(-i \mu_{n}\left|z-z_{s}\right|\right)$ with the $\mu_{n}$ determined by the corresponding $n$th positive zero of the denominator of the integrand (which may also depend on $\nu$ ). Consider now the special case where the bounded region is symmetric with respect to $z=0$, i.e. $h_{\min }=h_{\max }=h$. Applying the method of images then implies a substitution of each term $\exp \left(-i \mu_{n}\left|z-z_{s}\right|\right)$ by the expression

$$
\begin{gathered}
\left(e^{-i \mu_{n}\left|z-z_{s}\right|} H\left(z-z_{s}\right)+e^{-i \mu_{n}\left|z-z_{s}\right|} H\left(z_{s}-z\right)\right)[H(z+h)-H(z-h)] \\
\left.-\left(e^{-i \mu_{n}\left|z-z_{s}^{\prime}\right|} H\left(z-z_{s}\right)+e^{-i \mu_{n}\left|z-z_{s}^{\prime \prime}\right|} H\left(z_{s}-z\right)\right)[H(z+h)-H(z-h\rangle\} .59\right)
\end{gathered}
$$

with the image points $z_{s}^{\prime}=2 h-z_{s}, z_{s}^{\prime \prime}=-2 h-z_{s}$ and $H(z)$ the Heaviside step function. After some straightforward manipulations, (3.59) could also be written as

$$
\begin{aligned}
& +e^{-i \mu_{n}\left(z-z_{s}\right)} H\left(z-z_{s}\right)-e^{i \mu_{n}\left(z+z_{s}-2 h\right)} H\left(z-z_{s}\right) \\
& -e^{-i \mu_{n}\left(z-z_{s}\right)} H(z-h)+e^{i \mu_{n}\left(z+z_{s}-2 h\right)} H(z-h) \\
& +e^{i \mu_{n}\left(z-z_{s}\right)} H(z+h)-e^{-i \mu_{n}\left(z+z_{s}+2 h\right)} H(z+h) \\
& -e^{i \mu_{n}\left(z-z_{s}\right)} H\left(z-z_{s}\right)+e^{-i \mu_{n}\left(z+z_{s}+2 h\right)} H\left(z-z_{s}\right)
\end{aligned}
$$

For an application to extragalactic jets originating from accreting black hole systems however, it may be more appropriate to consider a bounded region specified by $h_{\min } \rightarrow 0$ and $h_{\max } \rightarrow \infty$. For such a simplified choice, the resultant Green's function satisfying homogeneous Dirichlet conditions on the boundary $z=0$ may be written as a superposition of the line source at $z_{s}$ and its image at $-z_{s}\left(z_{s}>0\right)$, i.e. one has

$$
e^{-i \mu_{n}\left|z-z_{s}\right|} H(z)-e^{-i \mu_{n}\left|z+z_{s}\right|} H(z) \text {. }
$$

\subsubsection{General Green's formula for Dirichlet boundary conditions}

The Green's formula for Dirichlet boundary conditions which gives the general solution of the transport equation in terms of the Green's function, has been derived by WJM 94 [see their Eqs. (3.24), (7.12)]. Let $f_{G b}^{\prime}\left(r, z, p^{\prime} ; r_{s}, z_{s}, p_{s}^{\prime}\right)$ be the Green's function solution for the bounded region under consideration satisfying homogeneous Dirichlet conditions at the 
surface $z=-h_{\min }, h_{\max }$ and at $r=r_{\text {in }}, r_{\text {out }}$. Then by using the steady state version of the Green's formula, the general solution of the steady state transport equation with source term $Q$ satisfying Dirichlet boundary conditions (not necessarily homogeneous ones) is given by

$$
\begin{aligned}
& f_{0}^{\prime}\left(r, z, p^{\prime}\right)=\int_{r_{\text {in }}}^{r_{\text {out }}} \mathrm{d} r_{s} \int_{-h_{\min }}^{h_{\max }} \mathrm{d} z_{s} \int_{0}^{\infty} \mathrm{d} p_{s}^{\prime} Q\left(r_{s}, z_{s}, p_{s}^{\prime}\right) f_{G b}^{\prime} \\
& -\int_{0}^{\infty} \mathrm{d} p_{s}^{\prime} \int_{-h_{\min }}^{h_{\max }} \mathrm{d} z_{s}\left[\kappa\left(r_{s}, p_{s}^{\prime}\right) \frac{\partial f_{G b}^{\prime}}{\partial r_{s}} f_{0}^{\prime}\left(r_{s}, z_{s}, p_{s}^{\prime}\right)\right]_{r_{s}=r_{\text {in }}}^{r_{s}=r_{\text {out }}} \\
& -\int_{0}^{\infty} \mathrm{d} p_{s}^{\prime} \int_{-h_{\min }}^{h_{\max }} \mathrm{d} z_{s}\left[\kappa\left(r_{s}, p_{s}^{\prime}\right)\left(\frac{\gamma^{2} \Omega^{2} r}{c^{2}} \frac{\left(p^{\prime}\right)^{2}}{p^{\prime}}\right) \frac{\partial f_{G b}^{\prime}}{\partial p_{s}^{\prime}} f_{0}^{\prime}\left(r_{s}, z_{s}, p_{s}^{\prime}\right)\right]_{r_{s}=r_{\text {in }}}^{r_{s}=r_{\text {out }}} \\
& -\int_{0}^{\infty} \mathrm{d} p_{s}^{\prime} \int_{r_{\text {in }}}^{r_{\text {out }}} \mathrm{d} r_{s}\left[\kappa\left(r_{s}, p_{s}^{\prime}\right) \frac{\partial f_{G b}^{\prime}}{\partial z_{s}} f_{0}^{\prime}\left(r_{s}, z_{s}, p_{s}^{\prime}\right)\right]_{z_{s}=-h_{\min }}^{z_{s}=h_{\max }},
\end{aligned}
$$

where the subscript $s$ at the bracket in line 3 indicates that the enclosed expression has to be evaluated at the position $\left(r_{s}, z_{s}, p_{s}^{\prime}\right)$. The derivatives of $f_{G b}^{\prime}$ should first be calculated at points inside $\mho$ and subsequently, the limit should be taken for $\partial \mho$. Eq. (3.62) represents the general Green's formula for the case where the boundary values of $f_{0}^{\prime}$ are not necessarily zero. However, if $f_{0}^{\prime}$ satisfies homogeneous Dirichlet boundary conditions, i.e. if one has $f_{0}^{\prime}\left(r_{\text {in }}, z, p^{\prime}\right)=f_{0}^{\prime}\left(r_{\text {out }}, z, p^{\prime}\right)=f_{0}^{\prime}\left(r,-h_{\text {min }}, p^{\prime}\right)=f_{0}^{\prime}\left(r, h_{\max }, p^{\prime}\right)=0$ at the surface, Eq. (3.62) simplifies to

$$
f_{0}^{\prime}\left(r, z, p^{\prime}\right)=\int_{r_{\text {in }}}^{r_{\text {out }}} \mathrm{d} r_{s} \int_{-h_{\min }}^{h_{\max }} \mathrm{d} z_{s} \int_{0}^{\infty} \mathrm{d} p_{s}^{\prime} Q\left(r_{s}, z_{s}, p_{s}^{\prime}\right) f_{G b}^{\prime} .
$$

\subsubsection{One- and two-dimensional Green's functions}

Even for the simplification $v_{z}=0$ and a simple galactic rotation law (i.e. $\Omega \propto 1 / r)$, the Green's function solution for the steady state transport equation Eq. (3.45) is not straightforward to evaluate (cf. WJM 94). However, we are especially interested in an analysis of the azimuthal effects of particle acceleration in rotating jet flows and may thus be content with a $z$-independent solution of the transport equation, i.e. with an investigation of the so-called one-dimensional Green's function which preserves much of the physics involved (cf. Morse \& Feshbach 1953, pp. 842-847). The one-dimensional Green's function may be found by integrating the two-dimensional ring source $Q$ (which depends on the space coordinates $r_{s}, z_{s}$ ) from $z_{s}=-\infty$ to $z_{s}=\infty$; i.e. the one-dimensional Green's function represents the Green's function 
solution for a steady state monoenergetic injection of particles from a infinite cylindrical surface parallel to the jet axis at radius $r_{s}$. Correspondingly, the general solution of the steady state transport equation may be calculated by taking the limit $h_{\min } \rightarrow \infty, h_{\max } \rightarrow \infty$ and replacing $f_{G b}^{\prime}$ by $f_{G}^{\prime}$ in Eq. (3.62). The connection between the two-dimensional and the onedimensional Green's function may be demonstrated more transparently using the solution Eq. (3.58) for the two-dimensional Green's function

$$
\begin{aligned}
f_{G}^{\prime}\left(r, z, \Phi ; r_{s}, z_{s}, \Phi_{s}\right)= & \frac{1}{4 \pi^{2}} \int_{-\infty}^{\infty} \mathrm{d} \mu \int_{-\infty}^{\infty} \mathrm{d} \nu \tilde{F}_{0}^{\prime}\left(r, r_{s} ; \mu, \nu\right) \\
& \times \exp \left[-i \mu\left(z-z_{s}\right)-i \nu\left(\Phi-\Phi_{s}\right)\right] .
\end{aligned}
$$

Integrating Eq. (3.64) over $z_{s}$ from $-\infty$ to $\infty$ yields the required onedimensional Green's function $f_{G, 1 D}\left(r, \Phi ; r_{s}, \Phi_{s}\right)$, i.e.

$$
f_{G, 1 D}^{\prime}=\int_{-\infty}^{\infty} \mathrm{d} z_{s} f_{G}^{\prime}\left(r, z, \Phi ; r_{s}, z_{s}, \Phi_{s}\right)
$$

Using the fourier integral theorem, i.e.

$$
f(x)=\frac{1}{2 \pi} \int_{-\infty}^{\infty} \mathrm{d} k \int_{-\infty}^{\infty} \mathrm{d} \eta \exp [i k(\eta-x)] f(\eta),
$$

Eq. (3.65) becomes

$$
f_{G, 1 D}^{\prime}=\frac{1}{2 \pi} \lim _{\mu \rightarrow 0} \int_{-\infty}^{\infty} \mathrm{d} \nu \tilde{F}_{0}^{\prime}\left(r, r_{s} ; \mu, \nu\right) \exp \left[-i \nu\left(\Phi-\Phi_{s}\right)\right] .
$$

We may arrive at the one-dimensional Green's function solution $f_{G, 1 D}^{\prime}$ however more immediately, by searching for a $z$-independent solution of the transport equation Eq. (3.45) from the outset (cf. WJM 94), using the source term $Q\left(r, p^{\prime}\right)=q_{0} \delta\left(r-r_{s}\right) \delta\left(p^{\prime}-p_{s}^{\prime}\right)$. For, integrating Eq. (3.45) over $z_{s}$ from $-\infty$ to $\infty$, using the expression Eq. (3.64) and taking the first $z$-derivative results in an integral of the form

$$
\begin{aligned}
\int_{-\infty}^{\infty} \mathrm{d} z_{s} & \int_{-\infty}^{\infty} \mathrm{d} \mu \int_{-\infty}^{\infty} \mathrm{d} \nu(-i \mu) \tilde{F}_{0}^{\prime}\left(r, r_{s} ; \mu, \nu\right) \\
& \times \exp \left[-i \mu\left(z-z_{s}\right)-i \nu\left(\Phi-\Phi_{s}\right)\right]
\end{aligned}
$$

which, applying the fourier integral theorem, can be shown to be zero. Similarly, the integral involving the second $z$-derivative vanishes. Thus, the one-dimensional Green's function we are seeking for, represents the solution of the modified transport equation 


$$
\begin{aligned}
\frac{\partial^{2} f_{0}^{\prime}}{\partial r^{2}} & +\left(\frac{1+\beta}{r}+[3+\alpha] \frac{\gamma^{2} \Omega^{2} r}{c^{2}}\right) \frac{\partial f_{0}^{\prime}}{\partial r} \\
& +\frac{\gamma^{4} r^{2}}{5 c^{2}}\left(1-v_{z}^{2} / c^{2}\right)\left(\frac{\mathrm{d} \Omega}{\mathrm{d} r}\right)^{2}\left([3+\alpha] \frac{\partial f_{0}^{\prime}}{\partial \Phi}+\frac{\partial^{2} f_{0}^{\prime}}{\partial \Phi^{2}}\right) \\
& =-\frac{q_{0}}{\kappa_{s} p_{s}^{\prime}} \delta\left(r-r_{s}\right) \delta\left(\Phi-\Phi_{s}\right)
\end{aligned}
$$

Since we are especially interested in the azimuthal evolution of the particle distribution function, we hereafter use Eq. (3.69) for our analysis of particle acceleration in rotating and shearing jet flows.

\subsection{Particle acceleration by rigidly rotating flows}

In the previous chapter we have investigated the acceleration of charged test particles at the base of a rigidly rotating jet magnetosphere. It has been shown there, that the maximum attainable Lorentz factor is limited by the breakdown of the bead-on-the-wire approximation which occurs in the vicinity of the light cylinder (see also Rieger \& Mannheim 2000a, 2001a). Here we supplementary consider the transport of relativistic particles further away from the base where the jet might be well-represented by a cylindrical model. To this end the evolution of the particle distribution is investigated by using the transport equation (3.69) for the case of rigid rotation $\Omega=\Omega_{0}$. Such rotation profiles could be related to dynamo action in the inner accretion disk creating a jet magnetosphere filled with disk plasma and rotating with the angular frequency of its foot points near the last marginally stable orbit around a spinning black hole (e.g. Camenzind 1996). In the case of solid body (uniform) rotation shearing in the background flow is absent since the fluid moves without internal distortions. For the case under consideration the one-dimensional Green's function corresponds to a $z$-independent solution of the steady state transport equation, i.e. to a solution of Eq. (3.69) which, using a constant $\Omega=\Omega_{0}$, simplifies to the purely spatial transport equation

$$
\frac{\partial^{2} f_{0}^{\prime}}{\partial r^{2}}+\left[\frac{1+\beta}{r}+(3+\alpha) \frac{\tilde{\Omega}_{0}^{2} r / c^{2}}{\left(1-\tilde{\Omega}_{0}^{2} r^{2} / c^{2}\right)}\right] \frac{\partial f_{0}^{\prime}}{\partial r}=Q_{0}
$$


where $\tilde{\Omega}_{0}$ is defined by

$$
\tilde{\Omega}_{0}=\frac{\Omega_{0}}{\sqrt{1-v_{z}^{2} / c^{2}}},
$$

while $Q_{0}=-q_{0} \delta\left(r-r_{s}\right) \delta\left(\Phi-\Phi_{s}\right) /\left(\kappa_{s} p_{s}^{\prime}\right)$, with $q_{0}$ given by Eq. (3.49), and where the diffusion coefficient is assumed to be of the form

$$
\kappa=\kappa_{\mathrm{o}}\left(\frac{p^{\prime}}{p_{\mathrm{o}}^{\prime}}\right)^{\alpha}\left(\frac{r}{r_{\mathrm{o}}}\right)^{\beta}
$$

where $\kappa_{\mathrm{o}}, p_{\mathrm{o}}^{\prime}$ and $\alpha, \beta$ are constants, cf. Eq. (3.8) and Eq. (3.37).

For rigid rotation Eq. (3.32) yields

$$
\begin{aligned}
H\left(r, p^{\prime}\right) & =p^{\prime 0} c \exp \left(-\int^{r} \mathrm{~d} r^{\prime} \frac{\gamma^{2} \Omega_{0}^{2} r^{\prime}}{c^{2}}\right) \\
& =p^{\prime 0} c \exp \left(\frac{1}{2} \ln \left[1-\Omega_{0}^{2} r^{\prime 2} / c^{2}-v_{z}^{2} / c^{2}\right]\right) \\
& =p^{\prime 0} c\left(1-\Omega_{0}^{2} r^{2} / c^{2}-v_{z}^{2} / c^{2}\right)^{1 / 2}=\frac{p^{\prime 0} c}{\gamma}
\end{aligned}
$$

where $\gamma$ denotes the Lorentz factor of the flow. If one generalizes the results given in appendix A.2 in order to include a $v_{z}$-component (i.e. if one considers the three-dimensional case), one may immediately recognize, that $H\left(r, p^{\prime}\right)$ is analogous to the Hamiltonian for a bead on a relativistically rotating wire, i.e. that $H\left(r, p^{\prime}\right)$ is a constant of motion (cf. Noether's theorem). The variable $H$ thus describes a balance between the centrifugal force and the inertia of the particle in the comoving frame (e.g. WJM 94) while Eq. (3.73) indicates that the ratio of the particle to the flow Lorentz factor is constant.

Since $H=H_{s}\left(r_{s}, p_{s}^{\prime}\right)$ is a constant of motion, the particle momentum $p^{\prime}$ in the comoving frame could be simply expressed as a function of the radial coordinate

$$
p^{\prime}(r)=m_{0} c \sqrt{\frac{H_{s}^{2}}{m_{0}^{2} c^{4}\left(1-\Omega_{0}^{2} r^{2} / c^{2}-v_{z}^{2} / c^{2}\right)}-1},
$$

with $m_{0}$ the rest mass of the particle [see also Fig. 3.1]. Hence, by solving Eq. (3.70) for the distribution function as a function of the radial coordinate, one may immediately calculate the corresponding particle momentum related to the position $r$. 


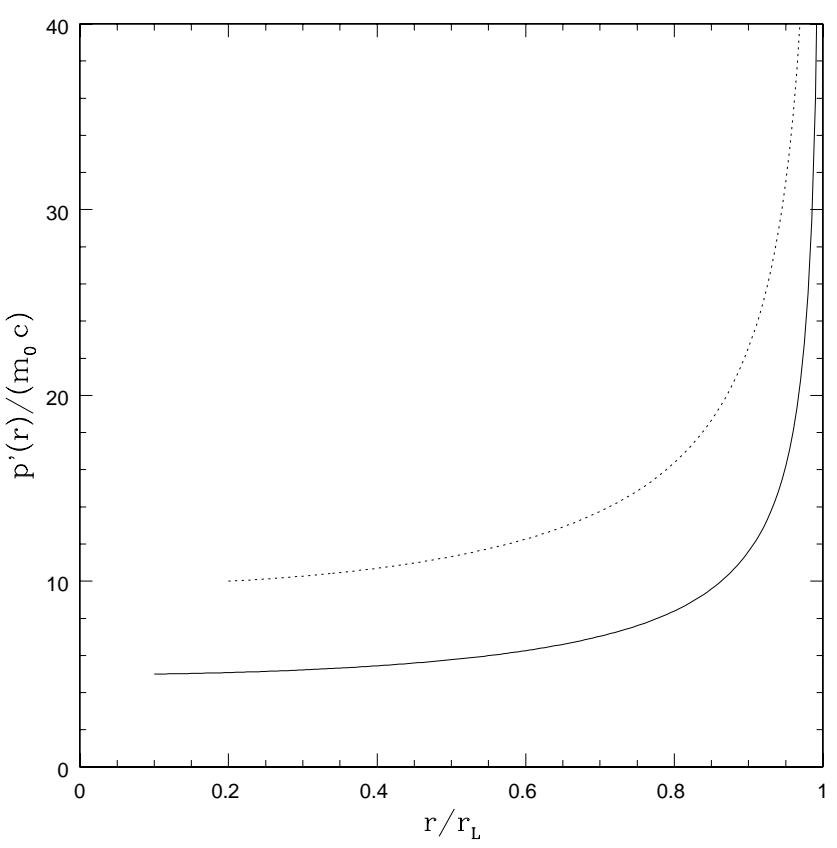

Figure 3.1: The particle momentum $p^{\prime}(r) /\left(m_{0} c\right)$ as a function of the radial coordinate for particles injected a.) at position $r_{s}=0.1 r_{\mathrm{L}}$, (where $r_{\mathrm{L}}=c / \tilde{\Omega}_{0}$, $v_{z}$ small) with initial Lorentz factor $\gamma_{s}=5$ (solid line) and b.) at position $r_{s}=0.2 r_{\mathrm{L}}$ with $\gamma_{s}=10$ (dotted line).

\subsubsection{Solution of the transport equation}

In order to solve Eq. (3.70) consider the substitution $y(r)=\partial f_{0}^{\prime} / \partial r$ for which the solution of the homogeneous part of Eq. (3.70) could be written as

$$
\begin{aligned}
y(r) & =\exp \left[-(1+\beta) \ln r+\frac{3+\alpha}{2} \ln \left(c^{2}-\tilde{\Omega}_{0}^{2} r^{2}\right)\right] \\
& =c^{3+\alpha} r^{-(1+\beta)}\left(1-\frac{\tilde{\Omega}_{0}^{2} r^{2}}{c^{2}}\right)^{(3+\alpha) / 2} .
\end{aligned}
$$

For the general solution $f_{h}^{\prime}$ of the homogeneous equation one therefore finds

$$
f_{h}^{\prime}=c_{1} \int^{r} y\left(r^{\prime}\right) \mathrm{d} r^{\prime}+c_{2}
$$

where $c_{1}$ and $c_{2}$ are constants. In the following, the integral of $y\left(r^{\prime}\right)$ over the interval $\mathrm{d} r^{\prime}$ is evaluated analytically for some special cases of interest:

- Consider first the case of a constant diffusion coefficient, i.e. $\alpha=\beta=0$. By substituting $u=\tilde{\Omega}_{0}^{2} r^{2} / c^{2}$ in the integrand, we arrive at an integral 
of the form

$$
\begin{aligned}
\int \frac{(1-u)^{3 / 2}}{u} \mathrm{~d} u & =\int \frac{\sqrt{1-u}}{u} \mathrm{~d} u-\int \sqrt{1-u} \mathrm{~d} u \\
& =2 \sqrt{1-u}-\ln \frac{1+\sqrt{1-u}}{1-\sqrt{1-u}}+\frac{2}{3}(1-u)^{3 / 2}
\end{aligned}
$$

Noting that $\ln [(1+\sqrt{1-u}) /(1-\sqrt{1-u})]=2 \ln [(1+\sqrt{1-u}) / \sqrt{u}]$, the general solution of the homogeneous equation could then be written as $f_{h}^{\prime}(r)=\tilde{c}_{1} y_{1}(r)+c_{2} y_{2}(r)$ where the two independent solutions $y_{1}, y_{2}$ are given by

$$
\begin{aligned}
y_{1}(r)= & \sqrt{1-\tilde{\Omega}_{0}^{2} r^{2} / c^{2}}\left(\frac{4}{3}-\frac{\tilde{\Omega}_{0}^{2} r^{2}}{3 c^{2}}\right) \\
& -\ln \frac{c\left(1+\sqrt{1-\tilde{\Omega}_{0}^{2} r^{2} / c^{2}}\right)}{\tilde{\Omega}_{0} r} \\
y_{2}(r) \equiv & 1 .
\end{aligned}
$$

For the appropriate Wronskian

$$
W(r) \equiv W\left(y_{1}(r), y_{2}(r)\right)=y_{1} \mathrm{~d} y_{2} / \mathrm{d} r-y_{2} \mathrm{~d} y_{1} / \mathrm{d} r
$$

one then simply has

$$
W(r)=-r^{-1}\left(1-\frac{\tilde{\Omega}_{0}^{2} r^{2}}{c^{2}}\right)^{3 / 2} .
$$

- In the case, where $\beta$ is negative, the integral may be expressed in terms of the incomplete Beta function. Defining the incomplete Beta function by (cf. Abramowitz \& Stegun 1965, p. 263)

$$
B(x, a, b)=\int_{0}^{x} x^{a-1}(1-x)^{b-1} \mathrm{~d} x,
$$

where $a, b>0$, writing $f_{h}^{\prime}(r)=\tilde{c}_{1} y_{1}(r)+c_{2} y_{2}(r)$ for the general solution of the homogeneous equation and using the substitution $u=\tilde{\Omega}_{0}^{2} r^{2} / c^{2}$, one finally may arrive at the system

$$
\begin{aligned}
y_{1}(r) & =\frac{1}{2}\left(\frac{\tilde{\Omega}_{0}}{c}\right)^{\beta} \times B\left(\frac{\tilde{\Omega}_{0}^{2} r^{2}}{c^{2}},-\frac{\beta}{2}, \frac{5+\alpha}{2}\right) \\
& \text { for } \alpha>-5, \text { and } \beta<0 \\
y_{2}(r) & \equiv 1 .
\end{aligned}
$$


Note, that now the solutions $y_{1}, y_{2}$ have been defined such that the appropriate Wronskian reduces to Eq. (3.80) for $\alpha=\beta=0$, i.e. we have

$$
W(r)=-r^{-(1+\beta)}\left(1-\frac{\tilde{\Omega}_{0}^{2} r^{2}}{c^{2}}\right)^{(3+\alpha) / 2}
$$

Generally, the solution space of the homogeneous part of Eq. (3.70) may be described by a set of two independent solutions, e.g. by the functions $y_{1}(r)$ and $y_{2}(r)$ with Wronskian $W(r)$ (where for an appropriate choice $y_{2}(r) \equiv$ 1). The general (one-dimensional Green's) solution of the inhomogeneous differential equation Eq. (3.70) with monoenergetic source term $Q_{0}$ defined above could then be written as (e.g. Morse \& Feshbach 1953, p. 530)

$$
\begin{aligned}
f_{0}^{\prime}\left(r, p^{\prime}\right)=y_{1}(r)\left[k_{1}\right. & \left.-\int^{r} \frac{Q_{0} y_{2}(r)}{W(r)} \mathrm{d} r\right] \\
& +y_{2}(r)\left[k_{2}+\int^{r} \frac{Q_{0} y_{1}(r)}{W(r)} \mathrm{d} r\right],
\end{aligned}
$$

where $k_{1}, k_{2}$ are integration constants specified by the boundary conditions. In the disk-jet scenario the accretion disk is usually assumed to supply the mass for injection into the jet, thus for simplicity one may consider a rather hollow jet structure (cf. Marcowith et al. 1995, Fendt 1997a; Subramanian, Becker \& Kazanas 1999) where the plasma motion in the azimuthal direction is restricted to a region $r_{\text {in }} \leq r \leq r_{\text {out }}<r_{\mathrm{L}}$ where $r_{\text {in }}$ denotes the jet inner radius, $r_{\text {out }}$ the relevant outer radius and $r_{\mathrm{L}}$ the light cylinder radius. The size of the inner radius $r_{\text {in }}$ may be chosen to be of the order of the radius of the last marginally stable orbit around a rotating black hole while the outer radius $r_{\text {out }}$ may be associated with the Alfvén radius derived in MHD scenarios. Particles are supposed to be injected at position $r_{\mathrm{s}}$ with initial momentum $p_{\mathrm{s}}^{\prime}$, where $r_{\text {in }}<r_{\mathrm{s}}<r_{\text {out }}$. By chosing the boundary conditions $f_{0}^{\prime}\left(r=r_{\text {in }}\right)=0$ and $f_{0}^{\prime}\left(r=r_{\text {out }}\right)=0$, the integration constants in Eq. (3.84) are determined by

$$
\begin{aligned}
k_{1} & =-k_{2} \frac{1}{y_{1}\left(r_{\text {in }}\right)} \text { and } \\
k_{2} & =\frac{\tilde{q}\left[y_{1}\left(r_{\text {out }}\right)-y_{1}\left(r_{\mathrm{s}}\right)\right]}{1-y_{1}\left(r_{\text {out }}\right) / y_{1}\left(r_{\text {in }}\right)},
\end{aligned}
$$

where

$$
\tilde{q}=-\frac{q_{0}}{\kappa_{s} p_{s}^{\prime} W\left(r_{s}\right)} \delta\left(\Phi-\Phi_{s}\right)
$$


with $q_{0}$ given by Eq. (3.49), i.e. $q_{0}=N_{s} /\left(8 \pi p_{s}^{\prime 2} r_{s}\right)$.

Therefore the (one-dimensional) Green's solution may be written as

$$
\begin{aligned}
f_{0}^{\prime}\left(r, p^{\prime} ; r_{s}, p_{s}^{\prime}\right) & =y_{1}(r)\left[k_{1} \theta\left(r-r_{\mathrm{s}}\right)+k_{1} \theta\left(r_{\mathrm{s}}-r\right)-\tilde{q} \theta\left(r-r_{\mathrm{s}}\right)\right] \\
& +\left[k_{2} \theta\left(r-r_{\mathrm{s}}\right)+k_{2} \theta\left(r_{\mathrm{s}}-r\right)+\tilde{q} y_{1}\left(r_{\mathrm{s}}\right) \theta\left(r-r_{\mathrm{s}}\right)\right]
\end{aligned}
$$

where $\theta(x)$ denotes the Heaviside step function. The delta function in Eq. (3.86) and Eq. (3.87) indicates that the particle momentum in the comoving frame is directly related to the relevant radial position by Eq. (3.74). In order to gain insight into the efficiency of the acceleration process one may introduce a spatial weighting function $N(r)$ defined by

$$
f_{0}^{\prime}\left(r, p^{\prime} ; r_{s}, p_{s}^{\prime}\right)=N(r) \delta\left(\Phi-\Phi_{s}\right)=N(r) H_{s} \delta\left(H-H_{s}\right) .
$$

The evolution of the function $N(r)$ is illustrated in Figs. 3.2-3.5, where we have plotted the normalized distribution function $N(r) / N\left(r_{\mathrm{s}}\right)$ as a function of the radial coordinate $r / r_{\mathrm{L}}$ for different initial conditions. Here, using Eq. (3.71), the radius $r_{\mathrm{L}}$ has been defined by $r_{\mathrm{L}}=c / \tilde{\Omega}_{0}$.

\subsubsection{Results and discussion}

The new function $N$ represents the spatial weighting function for the steadystate distribution of particles which where injected mono-energetically at position $r_{s}$ and distributed by diffusion over all relevant $r$. As one would expect, the solutions are characterized by a point of discontinuity at the position $r_{s}$ of the point source.

In order to understand the evolution in the case of rigidly rotating background flows, we might consider the original transport equation: For rigidly rotating flows of the form (3.13) the components $\sigma_{\alpha \beta}$ of the shear tensor Eq. (3.19-3.22) vanish, and thus, the viscous energization coefficient $\Gamma$, see Eq. (3.23), does also. Since the fluid four divergence also becomes zero, the only non-vanishing contribution responsible for a change of the particle energy in Eq. (3.2) is given by the acceleration vector term $\dot{u}_{\alpha} q^{\alpha}=\dot{u}_{1} q^{1}$ (cf. Eq. 3.25]). Eq. (3.18) then indicates, that the energy changes which take place are due solely to centrifugal effects. Hence, the increase of the particle energy with increasing radial distance, i.e. $p^{\prime}(r)$ [cf. Eq. (3.74)], is the same as in the test particle approach of chapter 2. For, if we generalize the results of chapter 2 to include a $v_{z}$-component, the test particle momentum $p^{\prime}$ in the comoving fluid frame could be written as

$$
p^{\prime}=\frac{m_{0} \dot{r}}{\sqrt{1-\Omega_{0}^{2} r^{2} / c^{2}-v_{z}^{2} / c^{2}-\dot{r}^{2} / c^{2}}}
$$




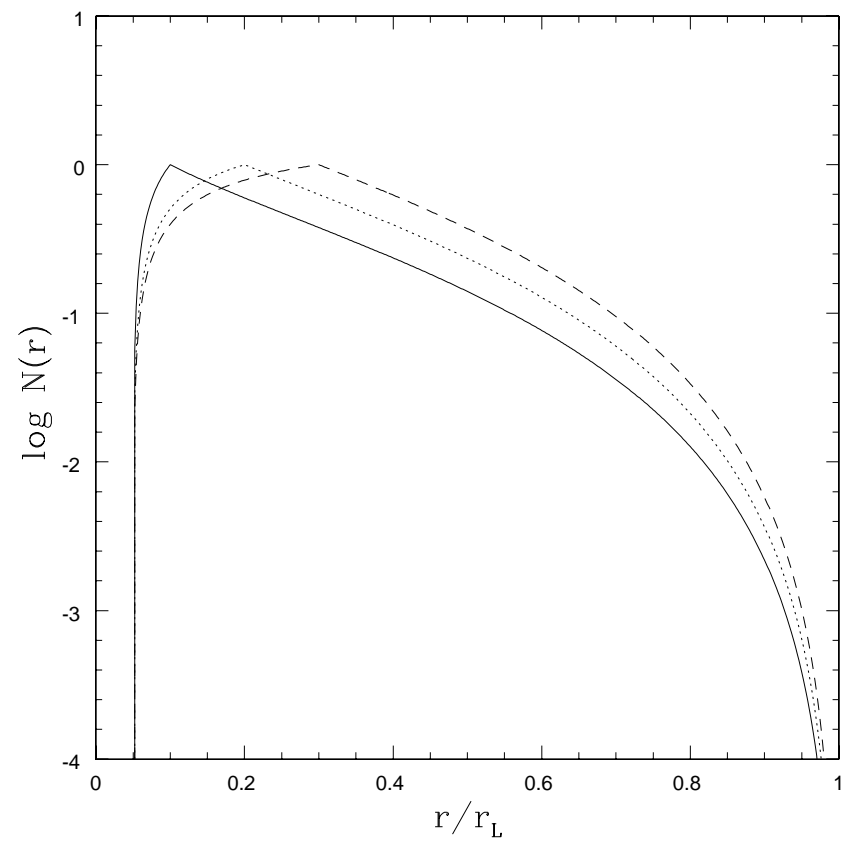

Figure 3.2: The normalized distribution function $N(r) / N\left(r_{\mathrm{s}}\right)$ for a constant diffusion coefficient (i.e. $\alpha=\beta=0$ ) using $r_{\text {in }}=0.05 r_{\mathrm{L}}, r_{\text {out }}=0.999 r_{\mathrm{L}}$ with particles injected at the positions $r_{\mathrm{s}} / r_{\mathrm{L}}=0.1$ (solid line), 0.2 (dotted line), 0.3 (dashed line).

$$
=\frac{m_{0} \dot{r}}{\sqrt{\tilde{m}}\left(1-\Omega_{0}^{2} r^{2} / c^{2}-v_{z}^{2} / c^{2}\right)},
$$

e.g. see Eq. (2.18), where $\tilde{m}$ might be chosen to be $\tilde{m}=m_{0}^{2} c^{4} / H_{s}^{2}$ (cf. Eq. 2.9]). Noting that

$$
\dot{r}=c \sqrt{\left(1-\Omega_{0}^{2} r^{2} / c^{2}-v_{z}^{2} / c^{2}\right)\left(1-\tilde{m}\left[1-\Omega_{0}^{2} r^{2} / c^{2}-v_{z}^{2} / c^{2}\right]\right)}
$$

cf. Eq. (2.8), we finally arrive at

$$
p^{\prime}(r)=m_{0} c \sqrt{\frac{H_{s}^{2}}{m_{0}^{2} c^{4}\left(1-\Omega_{0}^{2} r^{2} / c^{2}-v_{z}^{2} / c^{2}\right)}-1},
$$

which is indeed the same result as that given in Eq. (3.74).

Figs. 3.2, 3.3, 3.4 indicate that particle acceleration by rigidly rotating background flows might be very efficient. Related results have been already anticipated using the test particle approach in the bead-on-the-wire approximation (Gangadhara \& Lesch 1997; Rieger \& Mannheim 2000a, 2001a; see 


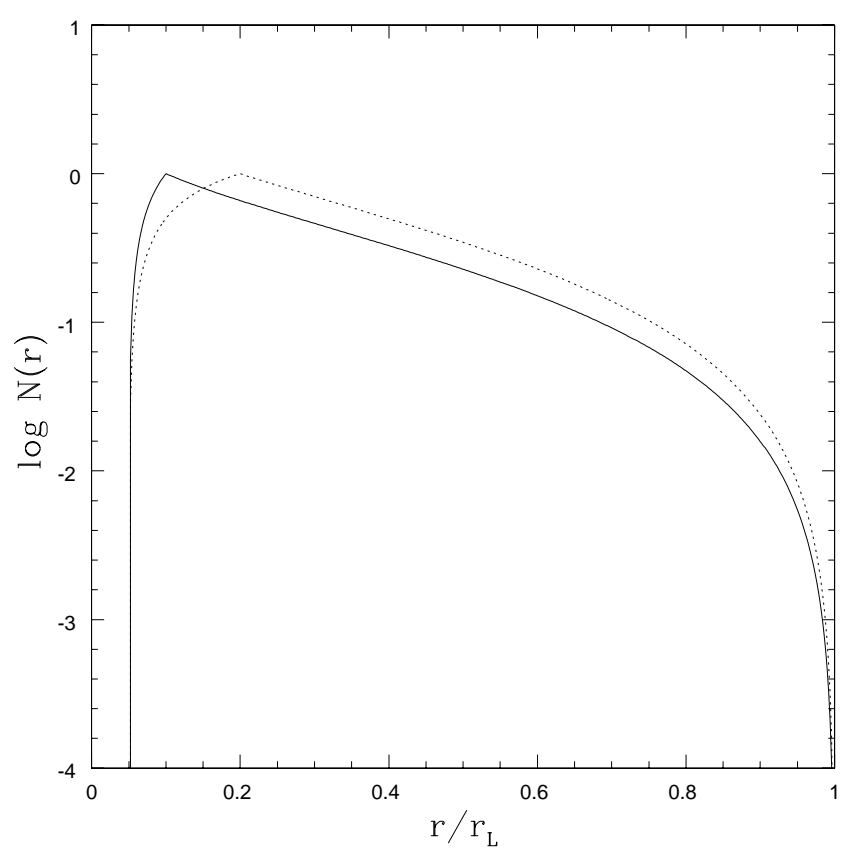

Figure 3.3: The normalized distribution function $N(r) / N\left(r_{\mathrm{s}}\right)$ for $\alpha=-2$ and $\beta=-0.01$, again using $r_{\text {in }}=0.05 r_{\mathrm{L}}, r_{\text {out }}=0.999 r_{\mathrm{L}}$ and different injection positions $r_{\mathrm{s}} / r_{\mathrm{L}}=0.1$ (solid line), 0.2 (dotted line).

chapter 2). However, as one would expect and as might be seen from Fig. 3.5, the acceleration efficiency, i.e. the transport of relativistic particles, essentially depends on the form of the diffusion coefficient

$$
\kappa=\kappa_{\mathrm{o}}\left(\frac{p^{\prime}}{p_{\mathrm{o}}^{\prime}}\right)^{\alpha}\left(\frac{r}{r_{\mathrm{o}}}\right)^{\beta}
$$

Especially, an increased efficiency is found, if the time interval for scattering $\tau_{c}$ is a monotonic decreasing function of $p^{\prime}$ (assuming only a weak spatial dependence), i.e. if scattering occurs more rapidly for the higher than for the lower energy particles (note that $\kappa \propto \tau_{c}$ for relativistic particles). In the case where $\tau_{c}$ is an increasing function of $p^{\prime}$ the efficiency may be reduced. However it should be noted that one requires $\left|v^{\prime} \tau_{c} / L_{f}\right| \ll 1$ (with $L_{f}$ the typical length scale for the evolution of $f_{0}^{\prime}$, e.g. Webb 1989) for the diffusion approximation to be valid which in some cases could be violated near the light cylinder. 


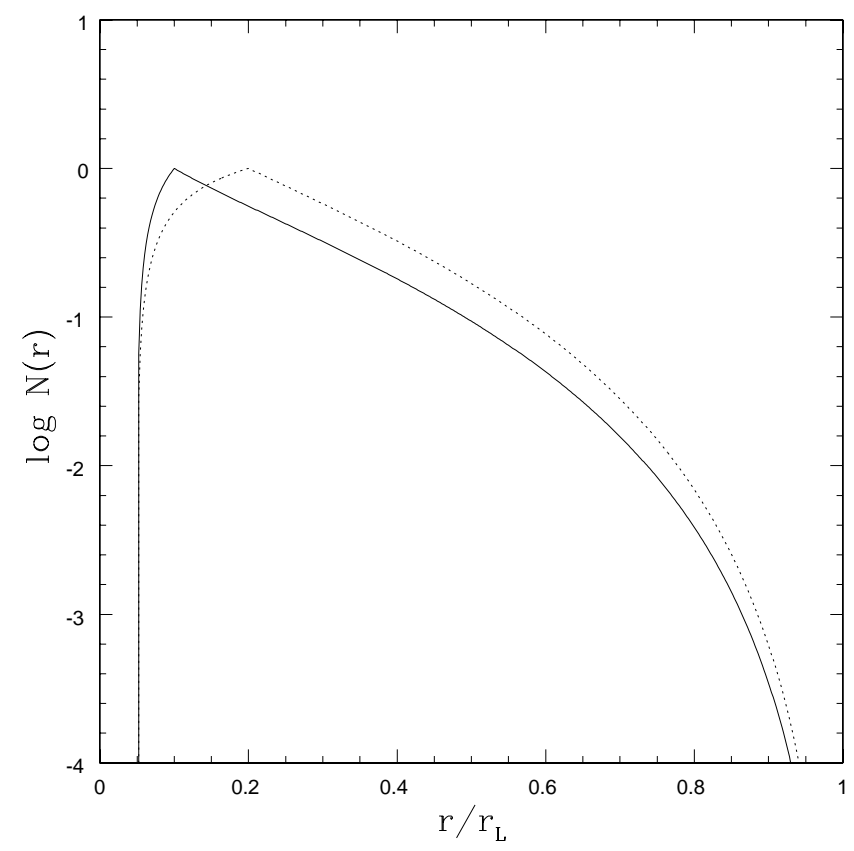

Figure 3.4: The normalized distribution function $N(r) / N\left(r_{\mathrm{s}}\right)$ for $\alpha=2$ and $\beta=-0.01$, again using $r_{\text {in }}=0.05 r_{\mathrm{L}}, r_{\text {out }}=0.999 r_{\mathrm{L}}$ and injection positions $r_{\mathrm{s}} / r_{\mathrm{L}}=0.1$ (solid line), 0.2 (dotted line).

\subsection{Particle acceleration by keplerian rotat- ing flows}

In this section we consider the acceleration of particles in a keplerian rotating background flow with $\Omega(r)=k r^{-3 / 2}, k=\sqrt{G M}$. Keplerian flow profiles might be related to jets or disk winds originating from the accretion disk around the black hole and dragging the keplerian rotation of the disk with them. A keplerian rotation profile may thus be regarded as one of the most realistic descriptions for rotating of source producing jets. Motivated by such arguments Lery \& Frank (2000) have recently investigated the structure and stability of astrophysical jets including keplerian rotation in the outermost part of the outflow and rigid rotation close to the axis (cf. also Hanasz, Sol \& Sauty 2000). They also studied the application to non-relativistic outflows from young stellar objects. One may thus eventually envisage a simple model where rigidly accelerated particles are subsequently injected into a keplerian flow profile.

For keplerian rotation both centrifugal and shear effects in the background flow are present. Using the transport equation (3.69), the one-dimensional 


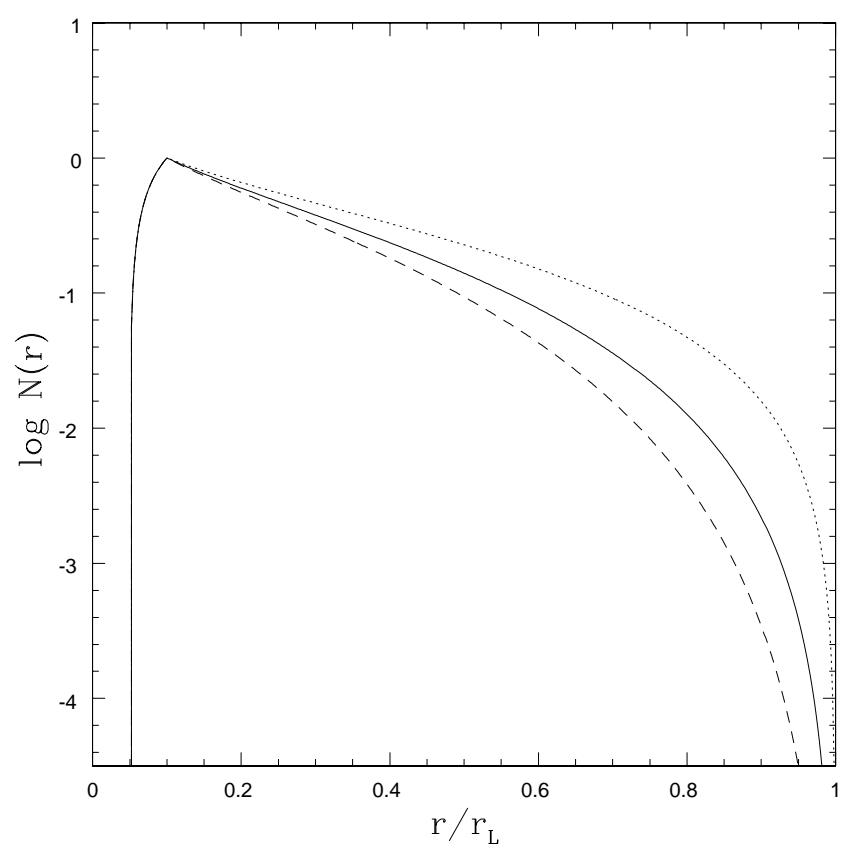

Figure 3.5: A comparison of the normalized distribution functions $N(r) / N\left(r_{\mathrm{s}}\right)$ for a different energy dependence of the diffusion coefficients, i.e. for $\alpha=\beta=0$ (solid line), $\alpha=-2, \beta=-0.01$ (dotted line) and $\alpha=2$, $\beta=-0.01$ (dashed line). $r_{\text {in }}=0.05 r_{\mathrm{L}}, r_{\mathrm{s}}=0.1 r_{\mathrm{L}}$ and $r_{\text {out }}=0.999 r_{\mathrm{L}}$ has been used for the calculations.

Green's function is the solution of the partial differential equation

$$
\begin{aligned}
\frac{\partial^{2} f_{0}^{\prime}}{\partial r^{2}} & +\left(\frac{1+\beta}{r}+[3+\alpha] \frac{\tilde{\gamma}(r)^{2} \tilde{\Omega}(r)^{2} r}{c^{2}}\right) \frac{\partial f_{0}^{\prime}}{\partial r} \\
& +\frac{\tilde{\gamma}(r)^{4} r^{2}}{5 c^{2}}\left(\frac{\mathrm{d} \tilde{\Omega}(r)}{\mathrm{d} r}\right)^{2}\left([3+\alpha] \frac{\partial f_{0}^{\prime}}{\partial \Phi}+\frac{\partial^{2} f_{0}^{\prime}}{\partial \Phi^{2}}\right)=Q_{0}
\end{aligned}
$$

where, in analogy to the previous section, $\tilde{\Omega}(r)$ is defined by

$$
\tilde{\Omega}(r)=\frac{\Omega(r)}{\sqrt{1-v_{z}^{2} / c^{2}}},
$$

while $\tilde{\gamma}(r)$ is defined by $\tilde{\gamma}(r)=\gamma(r) \sqrt{1-v_{z}^{2} / c^{2}}$, with $\gamma$ the Lorentz factor of the flow. The source term is given by

$$
Q_{0}=-\frac{q_{0}}{\kappa_{s} p_{s}^{\prime}} \delta\left(r-r_{s}\right) \delta\left(\Phi-\Phi_{s}\right)
$$


By applying Fourier transformation, i.e.

$$
F_{0}^{\prime}=\int_{-\infty}^{\infty} \mathrm{d} \Phi \exp [i \nu \Phi] f_{0}^{\prime}
$$

where the inverse Fourier transform is given by

$$
f_{0}^{\prime}=\frac{1}{2 \pi} \int_{-\infty}^{\infty} \mathrm{d} \nu \exp [-i \nu \Phi] F_{0}^{\prime}
$$

the transport equation (3.93) could be written as

$$
\begin{aligned}
\frac{\partial^{2} F_{0}^{\prime}}{\partial r^{2}} & +\frac{1}{r}\left(a_{1}+a_{2} \frac{\tilde{\gamma}(r)^{2}}{r}\right) \frac{\partial F_{0}^{\prime}}{\partial r} \\
& -\frac{\tilde{\gamma}(r)^{4}}{r^{3}}\left(i \nu a_{3}+a_{4} \nu^{2}\right) F_{0}^{\prime}=\tilde{Q}_{0} .
\end{aligned}
$$

where $\tilde{Q}_{0}$ denotes the Fourier transform of the source term Eq. (3.95), i.e.

$$
\tilde{Q}_{0}=-\frac{q_{0} \exp \left[i \nu \Phi_{s}\right]}{\kappa_{s} p_{s}^{\prime}} \delta\left(r-r_{s}\right)
$$

In Eq. (3.98) the abbreviations $a_{1}, a_{2}, a_{3}, a_{4}$ are defined by

$$
\begin{aligned}
& a_{1}=(1+\beta), \\
& a_{2}=(3+\alpha) \frac{G M}{\left(c^{2}-v_{z}^{2}\right)}, \\
& a_{3}=\frac{9}{20}(3+\alpha) \frac{G M}{\left(c^{2}-v_{z}^{2}\right)}, \\
& a_{4}=\frac{9}{20} \frac{G M}{\left(c^{2}-v_{z}^{2}\right)} .
\end{aligned}
$$

\subsubsection{Solution of the transport equation}

The analytical evaluation of Eq. (3.98) is complicated. However, a simple solution may be written down in the special case eventually being appropriate for the (outer) jet or wind solutions in AGN. For, consider the case of $r$ being so large that the rotational velocity becomes non-relativistic and the approximation

$$
\tilde{\gamma}(r)=\frac{1}{\sqrt{1-\frac{G M}{\left(c^{2}-v_{z}^{2}\right) r}}} \simeq 1
$$


holds. The Fourier transformed transport equation then simplifies to

$$
\frac{\partial^{2} F_{0}^{\prime}}{\partial r^{2}}+\frac{1}{r}\left(a_{1}+\frac{a_{2}}{r}\right) \frac{\partial F_{0}^{\prime}}{\partial r}-\frac{1}{r^{3}}\left(i \nu a_{3}+a_{4} \nu^{2}\right) F_{0}^{\prime}=\tilde{Q}_{0} .
$$

Using the substitution $y=a_{2} / r, a_{2} \neq 0$ (i.e. $\alpha \neq-3$ ), one arrives at

$$
y \frac{\partial^{2} F_{0}^{\prime}}{\partial y^{2}}+\left(2-a_{1}-y\right) \frac{\partial F_{0}^{\prime}}{\partial y}-\frac{i \nu a_{3}+a_{4} \nu^{2}}{a_{2}} F_{0}^{\prime}=0
$$

for the homogeneous part of Eq. (3.105), noting that relevant derivatives transform like

$$
\begin{aligned}
\frac{\partial F_{0}^{\prime}}{\partial r} & =-\frac{y^{2}}{a_{2}} \frac{\partial F_{0}^{\prime}}{\partial y} \\
\frac{\partial^{2} F_{0}^{\prime}}{\partial r^{2}} & =\frac{\partial}{\partial r}\left(\frac{\partial F_{0}^{\prime}}{\partial r}\right)=\frac{y^{4}}{a_{2}^{2}} \frac{\partial^{2} F_{0}^{\prime}}{\partial y^{2}}+2 \frac{y^{3}}{a_{2}^{2}} \frac{\partial F_{0}^{\prime}}{\partial y} .
\end{aligned}
$$

Eq. (3.106) is known in the literature as Kummer's equation (e.g. Abramowitz \& Stegun 1965, p. 504; Bateman 1964, p. 457). In the general case where $a_{2} \neq 0$ and $\left(2-a_{1}\right) \neq-n, n \in N_{0}$, the complete solution of this equation, i.e. of the homogeneous part of Eq. (3.105), may be written as

$$
F_{H}^{\prime}(r, \nu)=c_{1} f_{1}(r, \nu)+c_{2} f_{2}(r, \nu)
$$

where the functions $f_{1}, f_{2}$ are given by

$$
\begin{aligned}
& f_{1}(r, \nu)=M\left(\frac{i \nu a_{3}+a_{4} \nu^{2}}{a_{2}}, 2-a_{1}, \frac{a_{2}}{r}\right) \\
& f_{2}(r, \nu)=U\left(\frac{i \nu a_{3}+a_{4} \nu^{2}}{a_{2}}, 2-a_{1}, \frac{a_{2}}{r}\right) .
\end{aligned}
$$

Here, $M(a, b, y)$ and $U(a, b, y)$ denote the confluent hypergeometric functions (cf. Abramowitz \& Stegun 1965, pp. 504f; Buchholz 1953, pp. 1-9; Kamke 1944, pp. 643f), with $M(a, b, y)$ being characterized by the series representation

$$
M(a, b, y)=1+\frac{a}{1 ! b} y+\frac{a(a+1)}{2 ! b(b+1)} y^{2}+\frac{a(a+1)(a+2)}{3 ! b(b+1)(b+2)} y^{3}+\ldots
$$

while $U(a, b, y)$ is given by the series

$$
U(a, b, y)=\frac{\pi}{\sin \pi b}\left(\frac{M(a, b, y)}{\Gamma(1+a-b) \Gamma(b)}-y^{1-b} \frac{M(1+a-b, 2-b, y)}{\Gamma(a) \Gamma(2-b)}\right),
$$


with $\Gamma(x)$ the Gamma function.

$M(a, b, z)$ has a simple pole at $b=-n$ for $a \neq-m$ or for $a=-m$ if $m>n$, and is undefined for $b=-n, a=-m$ and $m \leq n$. $U(a, b, z)$, however, is defined even for $b \rightarrow \pm n$.

For the relevant Wronskian one has (e.g. Abramowitz \& Stegun 1965, p. 505)

$$
W(y) \equiv W(M(a, b, y), U(a, b, y))=-\Gamma(b) y^{-b} e^{y} / \Gamma(a)
$$

Thus, for the Wronskian $W(r) \equiv W\left(f_{1}, f_{2}\right)$ we find

$$
W(r)=\frac{\partial y}{\partial r} W(y)=\frac{a_{2}}{r^{2}} \Gamma\left(2-a_{1}\right)\left(\frac{a_{2}}{r}\right)^{-\left(2-a_{1}\right)} e^{a_{2} / r} / \Gamma(a(\nu)),
$$

where

$$
a(\nu)=\frac{i \nu a_{3}+a_{4} \nu^{2}}{a_{2}}
$$

In the particular case, where $a_{1}$ is not a positive integer, one may replace the confluent hypergeometric function $f_{2}(r, \nu)=U\left(a(\nu), 2-a_{1}, y(r)\right)$ by the hypergeometric function $f_{2}(r, \nu)=y(r)^{a_{1}-1} M\left(a(\nu)+a_{1}-1, a_{1}, y(r)\right)$ (cf. Bateman 1964, p. 457; Morse \& Feshbach 1953, p. 605), which then also represents a (quite simplier) second linearly independent solution to the Kummer's equation Eq. (3.106), with Wronskian now being given by $W(y)=\left(a_{1}-1\right) y(r)^{a_{1}-2} e^{y(r)}$.

For the general solution of the inhomogeneous fourier equation (3.98) one finds [cf. Eq. (3.84)]

$$
\begin{aligned}
F_{0}^{\prime}(r, \nu) & =f_{1}(r, \nu)\left[k_{1} \theta\left(r-r_{\mathrm{s}}\right)+k_{1} \theta\left(r_{\mathrm{s}}-r\right)+\frac{\tilde{q}}{W\left(r_{\mathrm{s}}\right)} f_{2}\left(r_{\mathrm{s}}, \nu\right) \theta\left(r-r_{\mathrm{s}}\right)\right] \\
& +f_{2}(r, \nu)\left[k_{2} \theta\left(r-r_{\mathrm{s}}\right)+k_{2} \theta\left(r_{\mathrm{s}}-r\right)-\frac{\tilde{q}}{W\left(r_{\mathrm{s}}\right)} f_{1}\left(r_{\mathrm{s}}, \nu\right) \theta\left(r-r_{\mathrm{s}}\right)\right],
\end{aligned}
$$

where $\tilde{q}$ has been defined by

$$
\tilde{q}=q_{0} \frac{\exp \left[i \nu \Phi_{s}\right]}{\kappa_{s} p_{s}^{\prime}}
$$

with $q_{0}$ given by Eq. (3.49) and where $k_{1}, k_{2}$ are integration constants specified by the boundary conditions. For homogeneous Dirichlet conditions at the boundaries $r_{\text {in }}$ and $r_{\text {out }}$, i.e. $F_{0}\left(r_{\text {in }}, \nu\right)=F_{0}\left(r_{\text {out }}, \nu\right)=0$ with $r_{\text {in }}<r<r_{\text {out }}$ and $r_{\text {in }}<r_{s}<r_{\text {out }}$ the integration constants are fixed (for each $\nu$ ) and given 
by

$$
\begin{aligned}
& k_{2}(\nu)=\frac{\tilde{q}}{W\left(r_{\mathrm{s}}\right)} f_{1}\left(r_{\mathrm{in}}, \nu\right) \frac{f_{2}\left(r_{\mathrm{out}}, \nu\right) f_{1}\left(r_{\mathrm{s}}, \nu\right)-f_{1}\left(r_{\mathrm{out}}, \nu\right) f_{2}\left(r_{\mathrm{s}}, \nu\right)}{f_{2}\left(r_{\mathrm{out}}, \nu\right) f_{1}\left(r_{\mathrm{in}}, \nu\right)-f_{1}\left(r_{\text {out }}, \nu\right) f_{2}\left(r_{\mathrm{in}}, \nu\right)} \\
& k_{1}(\nu)=-\frac{f_{2}\left(r_{\mathrm{in}}, \nu\right)}{f_{1}\left(r_{\mathrm{in}}, \nu\right)} k_{2} .
\end{aligned}
$$

Fourier inversion now yields the required (one-dimensional) Green's function

$$
f_{0}^{\prime}\left(r, \Phi ; r_{s}, \Phi_{s}\right)=\frac{1}{2 \pi} \int_{-\infty}^{\infty} \mathrm{d} \nu \exp [-i \nu \Phi] F_{0}^{\prime}(r, \nu)
$$

with $F_{0}^{\prime}(r, \nu)$ given by Eq. (3.117). As may be obvious from the foregoing investigation this fourier inversion is not easy to evaluate, not even using numerical methods. However, in order to cope with the integration, one may consider the following substitution $(\alpha \neq-3)$

$$
\omega=\frac{3}{\sqrt{20(3+\alpha)}}\left(\nu+\frac{3+\alpha}{2} i\right),
$$

for which one finds [cf. Eq. (3.116) and Eqs. (3.100)-(3.103)]

$$
a(\nu)=\omega^{2}+\sigma^{2}
$$

where $\sigma$ is defined by $\sigma=3 \sqrt{3+\alpha} / \sqrt{80}$.

Performing the substitution in Eq. (3.121), one arrives at an integral with the path of integration $A$ now in the complex plane, i.e. parallel to the real axis at a distance $3(3+\alpha) i /[2 \sqrt{20(3+\alpha)}]$, extending from $-R$ to $R$ with $R \rightarrow \infty$. We may close the path by choosing a rectangular contour $C$ which consists of the stated parallel $A$, the real axis, and the outer lines $B_{1}, B_{2}$ parallel to the imaginary axis at $R$ and $-R$, respectively. For the relevant ranges of $\alpha\left(\alpha \neq-3 ; 2-a_{1} \neq-n\right)$ of interest, the integrand has no poles within the region bounded by $C$ and thus, by virtue of Cauchy's integral theorem, the value of the contour integral around $C$ sums to zero. For $R \rightarrow \infty$ the integrals over $B_{1}$ and over $B_{2}$ vanish as might be shown by using asymptotic expansion formulas for the integrand. Noting that by means of Euler's equation $e^{i x}=\cos x+i \sin x$ we have

$$
\int_{-\infty}^{\infty} e^{-i x} f\left(x^{2}\right) \mathrm{d} x=2 \int_{0}^{\infty} f\left(x^{2}\right) \cos x \mathrm{~d} x
$$


and collecting all relevant expressions together, one finally may arrive at the integral $\left(2-a_{1} \neq-n ; \alpha \neq-3\right)$

$$
\begin{aligned}
& f_{0}^{\prime}\left(r, p^{\prime} ; r_{\mathrm{s}}, p_{s}^{\prime}\right)=g\left(r_{s}, p_{s}^{\prime}, \alpha, \beta\right) \exp \left[-\frac{3+\alpha}{2}\left(\Phi-\Phi_{s}\right)\right] \\
& \int_{0}^{\infty} \mathrm{d} \omega \cos \left(\frac{\sqrt{20(3+\alpha)}}{3} \omega\left(\Phi-\Phi_{s}\right)\right) \\
& \times \Gamma\left(\omega^{2}+\sigma^{2}\right)\left[f_{1}\left(r, \omega^{2}\right) f_{2}\left(r_{\text {out }}, \omega^{2}\right) \frac{h\left(r_{\text {in }}, r_{\mathrm{s}} ; \omega^{2}\right)}{h_{N}\left(r_{\text {in }}, r_{\text {out }} ; \omega^{2}\right)}\right. \\
& \left.+f_{2}\left(r, \omega^{2}\right) f_{1}\left(r_{\text {out }}, \omega^{2}\right) \frac{h\left(r_{\mathrm{s}}, r_{\text {in }} ; \omega^{2}\right)}{h_{N}\left(r_{\text {in }}, r_{\text {out }} ; \omega^{2}\right)}\right] \\
& \text { for } r>r_{\mathrm{s}} \\
& =g\left(r_{s}, p_{s}^{\prime}, \alpha, \beta\right) \exp \left[-\frac{3+\alpha}{2}\left(\Phi-\Phi_{s}\right)\right] \\
& \int_{0}^{\infty} \mathrm{d} \omega \cos \left(\frac{\sqrt{20(3+\alpha)}}{3} \omega\left(\Phi-\Phi_{s}\right)\right) \\
& \times \Gamma\left(\omega^{2}+\sigma^{2}\right)\left[f_{1}\left(r, \omega^{2}\right) f_{2}\left(r_{\text {in }}, \omega^{2}\right) \frac{h\left(r_{\text {out }}, r_{\mathrm{s}} ; \omega^{2}\right)}{h_{N}\left(r_{\text {in }}, r_{\text {out }} ; \omega^{2}\right)}\right. \\
& \left.+f_{2}\left(r, \omega^{2}\right) f_{1}\left(r_{\text {in }}, \omega^{2}\right) \frac{h\left(r_{\mathrm{s}}, r_{\text {out }} ; \omega^{2}\right)}{h_{N}\left(r_{\text {in }}, r_{\text {out }} ; \omega^{2}\right)}\right] \\
& \text { for } r<r_{\mathrm{s}}
\end{aligned}
$$

where we have introduced the following abbreviations

$$
\begin{aligned}
g\left(r_{s}, p_{s}^{\prime}, \alpha, \beta\right) & =\frac{2 q_{0} \sqrt{5(3+\alpha)}}{3 \pi \kappa_{s} p_{s}^{\prime} \Gamma\left(2-a_{1}\right)} r_{\mathrm{s}}\left(\frac{a_{2}}{r_{\mathrm{s}}}\right)^{1-a_{1}} \exp \left(\frac{-a_{2}}{r_{\mathrm{s}}}\right) \\
f_{1}\left(r, \omega^{2}\right) & =M\left(\omega^{2}+\sigma^{2}, 2-a_{1}, a_{2} / r\right), \\
f_{2}\left(r, \omega^{2}\right) & =U\left(\omega^{2}+\sigma^{2}, 2-a_{1}, a_{2} / r\right), \\
h\left(r_{\mathrm{in}}, r_{\mathrm{s}} ; \omega^{2}\right) & =f_{1}\left(r_{\mathrm{in}}, \omega^{2}\right) f_{2}\left(r_{\mathrm{s}}, \omega^{2}\right)-f_{2}\left(r_{\mathrm{in}}, \omega^{2}\right) f_{1}\left(r_{\mathrm{s}}, \omega^{2}\right), \\
h_{N}\left(r_{\mathrm{in}}, r_{\text {out }} ; \omega^{2}\right) & =f_{2}\left(r_{\text {out }}, \omega^{2}\right) f_{1}\left(r_{\mathrm{in}}, \omega^{2}\right)-f_{1}\left(r_{\text {out }}, \omega^{2}\right) f_{2}\left(r_{\mathrm{in}}, \omega^{2}\right),
\end{aligned}
$$

with $q_{0}$ given by Eq. (3.49). A further simplification may be exploited in the limit $r_{\text {out }} \rightarrow \infty$ in which case $a_{2} / r_{\text {out }} \rightarrow 0$ and thus, $f_{1}\left(r_{\text {out }}, \omega^{2}\right)=$ 1 and $f_{2}\left(r_{\text {out }}, \omega^{2}\right)=\Gamma\left(a_{1}-1\right) / \Gamma\left(a_{1}-1+\omega^{2}+\sigma^{2}\right)$ if $0<\left(2-a_{1}\right)<1$ (cf. Abramowitz \& Stegun 1965). Eq. (3.125) is evaluated using numerical methods (cf. Wolfram 1996). $f_{1}\left(r, \omega^{2}\right)$ and $f_{1}\left(r, \omega^{2}\right)$ are bounded for $\omega=$ 
0 while the integrand behaves well enough when $\omega \rightarrow \infty$ to allow for a numerical evaluation. As an example, the run of the integrand as a function of $\omega$ is shown in Fig. 3.6 for $r_{\text {out }}=1000 r_{\mathrm{ms}}, r_{\mathrm{in}}=10 r_{\mathrm{ms}}, r_{\mathrm{s}}=20 r_{\mathrm{ms}}$, $r=40 r_{\mathrm{ms}}, \alpha=1, \beta=0$ and $p^{\prime} / p_{s}^{\prime}=10$. Here, the radius $r_{\mathrm{ms}}$ has been defined such that the relevant quotient $a_{2} / r$ becomes independent of $v_{z}$ if one writes $r$ in units of $r_{\mathrm{ms}}$, i.e.

$$
r_{\mathrm{ms}}=\frac{1}{1-v_{z}^{2} / c^{2}} r_{\mathrm{sw}}=\frac{G M}{c^{2}-v_{z}^{2}},
$$

where $r_{\mathrm{sw}}$ denotes the half of the Schwarzschild radius.

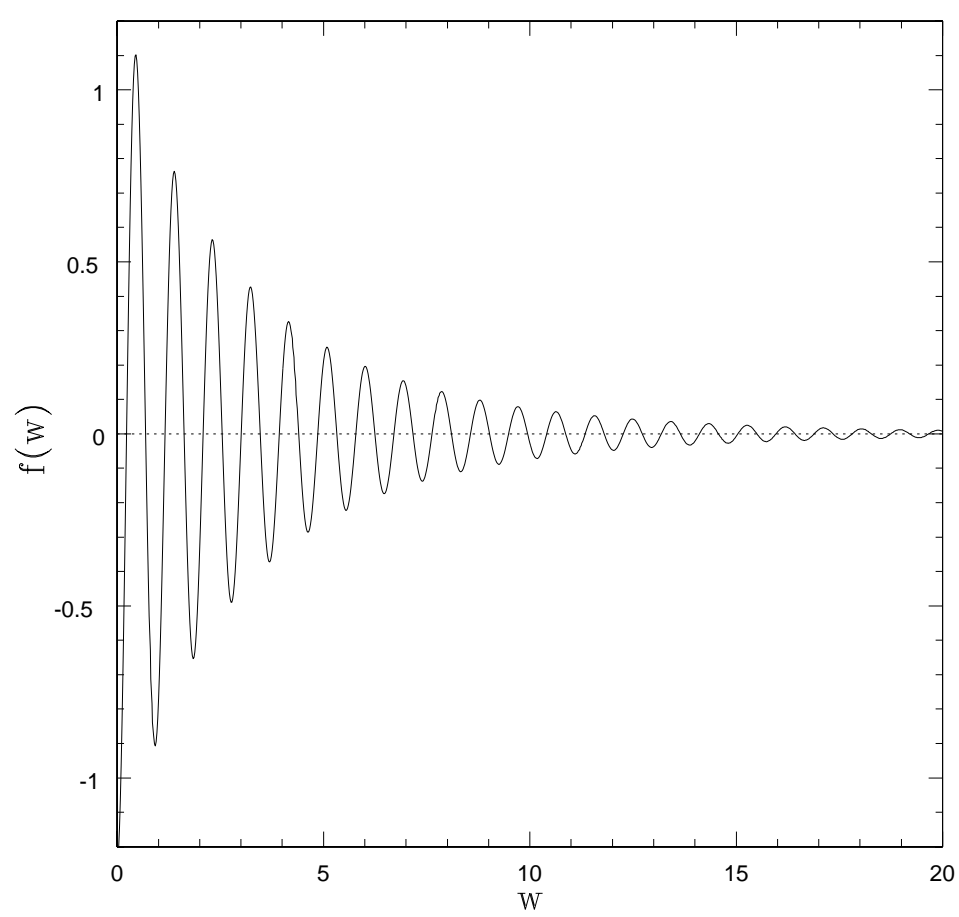

Figure 3.6: Illustrating the solution for the fourier transformed equation, i.e. the run of the integrand given by Eq. (3.125) as a function of $\omega$.

\subsubsection{Results and discussion}

For the special flow profiles considered in this chapter, the fluid four divergence vanishes. Hence, energy changes due to adiabatic compression or expansion of the fluid are absent and particle acceleration therefore (only) occurs as a consequence of the centrifugal and differential shear effects. As 
was shown above, the shear effects are additionally absent if we consider rigidly rotating background flows, i.e. for such flow profiles energy changes are due solely to the centrifugal force. In the case of keplerian rotation generally both, shear and centrifugal effects are present. However, estimating the relative strength of the contribution by shear and centrifugal effects in the present application, one obtains a scaling $r^{-2}$ for the centrifugal and up to $r^{-1.5}$ for the shear effects (cf. also WJM 94). Thus, for the approximation above [i.e. Eq. (3.104)], shear effects may eventually be dominant. This is especially illustrated in Fig. 3.7, where we have plotted the logarithmic

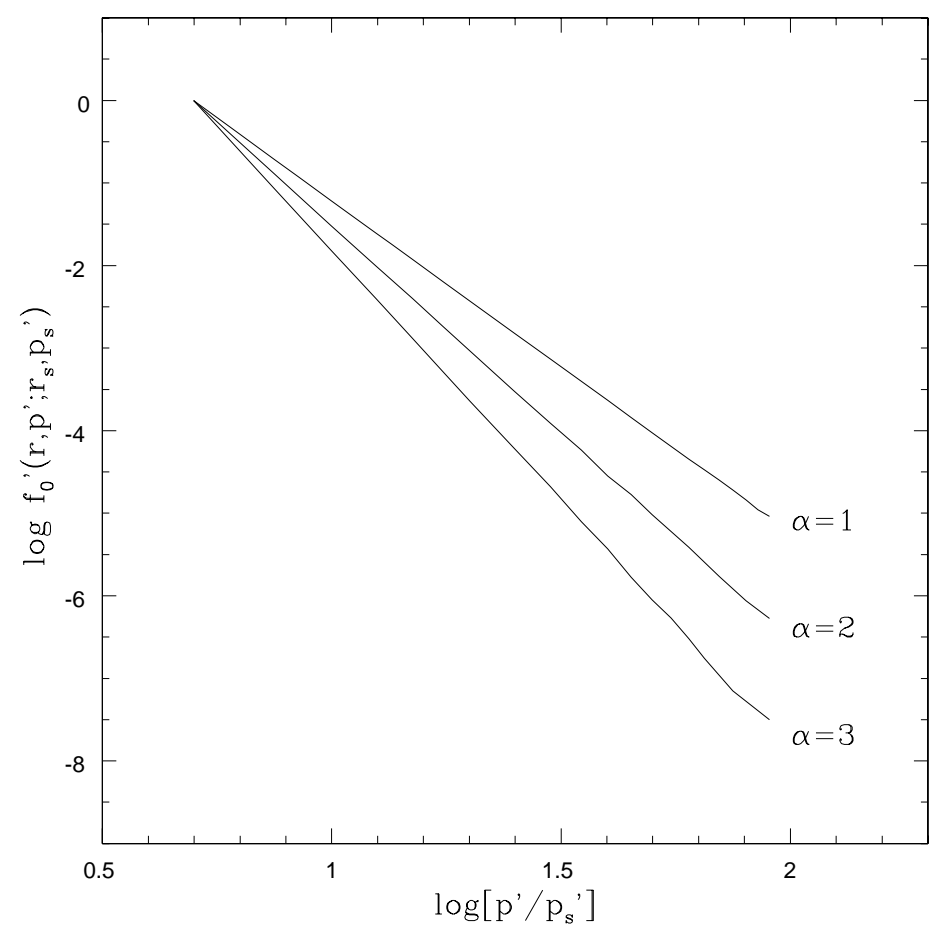

Figure 3.7: The momentum-dependence of the (normalized) distribution function $f_{0}^{\prime}$ for keplerian rotation using $\beta=0$, calculated for $\alpha=1,2,3$ at fixed $r=40 r_{\mathrm{ms}}$. Boundary and injection conditions have been specified as $r_{\mathrm{in}}=10 r_{\mathrm{ms}}, r_{\mathrm{out}}=1000 r_{\mathrm{ms}}, r_{\mathrm{s}}=20.0 r_{\mathrm{ms}}$.

of the (normalized) particle distribution function $f_{0}^{\prime}$ using $\beta=0$ (i.e. no spatial dependence for the collision time). In order to evade possible numerical complications near the injection momentum $p_{s}^{\prime}$, the distributions have been calculated for values $p^{\prime} / p_{s}^{\prime} \geq 5$. Obviously, excellent powerlaw-type momentum spectra are recovered, e.g. $f_{0}^{\prime} \propto p^{\prime-4}$ for $\alpha=1.0, f_{0}^{\prime} \propto p^{\prime-5}$ for $\alpha=2$ and $f_{0}^{\prime} \propto p^{\prime-6}$ for $\alpha=3$, suggesting $f_{0}^{\prime} \propto p^{-(3+\alpha)}$ for the range con- 
sidered. The existence of simple power-law momentum solutions, as derived by Berezhko \& Krymskii (1981), is thus confirmed. Using the Boltzmann kinetic equation for a collisionless plasma with shear flow $U(y) \vec{e}_{x}$ and a simple BGK term, they found the shear acceleration to give rise to a powerlaw momentum spectrum for the steady state comoving particle distribution $n^{\prime}\left(r, p^{\prime}\right) \propto p^{\prime 2} f_{0}^{\prime} \propto p^{-(1+\alpha)}$ if the collision time $\tau_{c}$ depends on momentum as $\tau_{c} \propto p^{\prime \alpha}$. If the momentum index $\alpha$ however, is smaller than zero, i.e. $\alpha<0$, an exponential spectrum might be developed. In their approach however, only a momentum dependence of the scattering time $\tau_{c}$ has been considered. Besides providing the possibility for a detailed consideration of centrifugal effects, the derivation presented here has the additional advantage also to allow for a radial dependence of $\tau_{c}$ (e.g. $\tau_{c} \propto r^{\beta} p^{\alpha}, \beta \neq 0$ ). An example is illustrated in Fig. 3.8 revealing a slightly steepening of the momentum distribution if $\tau_{c}$ is a decreasing function of the radial distance $r$. This may be understood as a consequence of the shear (and also the centrifugal) effects to be more important at smaller radii (i.e. they increase with decreasing radii), but the mean time between scattering events there to be enlarged as well.

The generation of a power law particle distribution is usually considered to be of wide astrophysical relevance. If we convolve, for example, a power law electron distribution with index $-(1+\alpha)$ with the synchrotron Green's function, a power law for the volume spectral emissivity $j_{\nu}$ is obtained, i.e. $j_{\nu} \propto \nu^{-\alpha / 2}$. For many synchrotron sources a spectral index in the range of $0.5-0.75$ is observed. Using the above power law, $\alpha$ would then required to be in the range $(1-1.5)$.

\subsection{Conclusion}

Observational and theoretical arguments suggest that astrophysical jets should exhibit intrinsic rotation of material perpendicular to the jet axis. In the present study we therefore have investigated the acceleration of energetic particles by centrifugal and viscous shear effects in a collisionless rotating background flow. Our investigation utilizes a simple version of the relativistic (steady-state) particle transport equation derived by Webb (1989) (cf. also Webb et al. 1994) assuming that the diffusion approximation holds. The results indicate that under special conditions the acceleration of particles by rotating jet flows may be a very efficient mechanism for the production of high energy particles.

In the present study we have examined the influence of rigid and keplerian 


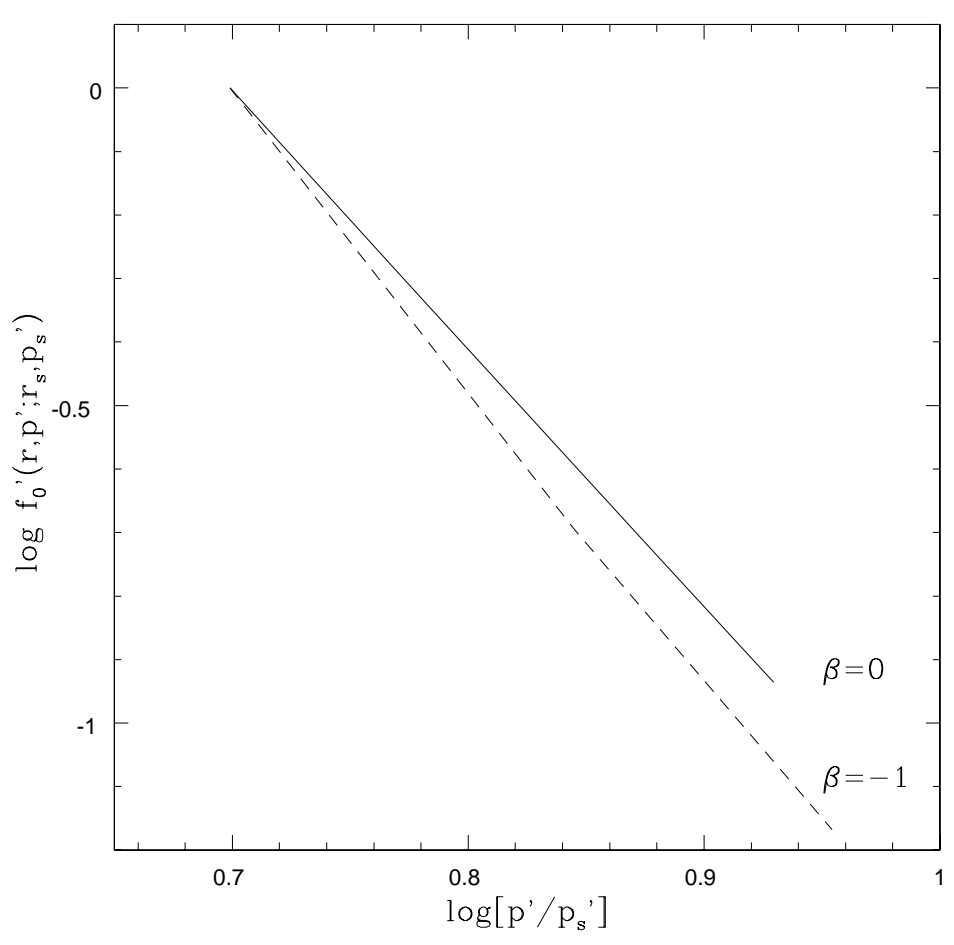

Figure 3.8: The momentum-dependence for the (normalized) distribution function $f_{0}^{\prime}$ using $\alpha=1$, calculated for $\beta=0,-1$ at fixed $r=40 r_{\mathrm{ms}}$. Boundary and injection conditions have been specified as $r_{\mathrm{in}}=10 r_{\mathrm{ms}}, r_{\mathrm{out}}=$ $1000 r_{\mathrm{ms}}, r_{\mathrm{s}}=20 r_{\mathrm{ms}}$.

rotation profiles on the steady-state particle distribution. Such rotation profiles are particularly interesting if one considers, for example, a simple jet model where rigidly accelerated particles (in the inner part of the jet) are subsequently injected into a keplerian flow (in the outer part of the jet). For the considered rotation profiles, we may distinguish the acceleration of particles by centrifugal and by shear effects.

In the case of rigid rotation, shearing in the background flow is absent and the energy changes are solely due to the centrifugal effects. Analogous to the test particle approach of chapt. 2, particles gain energy due to the centrifugal force while moving outwards by diffusion until the rigid rotation of the background flow is suppressed in the immediate vicinity of the light cylinder. The position of the decoupling point then limits the maximum energy attainable by rigid rotation, similar to the breakdown of 
the bead-on-the-wire approximation in the test particle approach.

In the case of keplerian rotation profiles shearing in the differentially rotating background flow is present and particle energy changes are in general due to both, centrifugal and viscous shear effects. To allow of a simple analytical treatment, we have considered the case of non-relativistic keplerian rotation eventually being appropriate for the outer jet solutions where the shear effects dominate. We confirm the formation of powerlaw-type momentum spectra $n\left(r, p^{\prime}\right) \propto p^{-(1+\alpha)}$ if the collision time depends on momentum as $\tau_{c} \propto p^{\prime \alpha}, \alpha>0$ (cf. Berezhko \& Krymskii 1981). If there is a more complex interplay between centrifugal and shear effects, however, one expects that the spectrum may become flatter with increasing rotational velocity.

Our present results reveal the power of shear and centrifugal acceleration in intrinsically rotating astrophysical jets. The operation of such a mechanism may not only provide the high energy particles required for efficient Fermi-type acceleration, also generally expected to occur in relativistic jets (e.g. Drury 1983, Kirk et al. 1994). But probably more important, the analysed mechanism could be of particular relevance for an explanation of the continuous emission observed from the jets of several AGN (e.g. for 3C273; M87; PKS 0521-36; cf. Meisenheimer, Yates, Röser 1997; Jester et al. 2001). For, since intrinsic jet rotation is expected in the AGN setting, efficient acceleration might occur over the whole relevant jet length. Thus, in contrast to shock acceleration, this mechanism is generally not constrained to a localized region. Recent observations indeed indicate that the radiating particles could be widely distributed such that the optical emission from radio jets, for example, is not confined to bright individual knots (cf. Meisenheimer, Röser, Schlötelburg 1996; Meisenheimer, Yates, Röser 1997). In addition to diffusive shock acceleration, an extended acceleration mechanism associated with velocity shear has thus been suggested by Meisenheimer, Yates \& Röser (1997). Meanwhile, the need of an extended acceleration mechanism is strengthened by recent HST observations of the jet in 3C273 showing no evidence for localized acceleration or emission zones throughout the jet (Jester et al. 2001).

The present approach therefore appears to be quite fruitful. We would like to mention however, that the validity of the underlying transport equation imposes the restriction that scattering is strong enough to allow for the diffusion approximation. This may represent a serious limitation if highly anisotropic distributions are expected, as for example near shock fronts. Hence in such 
cases the diffusion approximation is expected to break down. Also, in the situation where the individual particle's motion is dominated by the Lorentz force of the background magnetic field, the gyrofrequency of the charged particle may become a large quantity and thus, the distribution function may be regarded as nearly independent of the gyration phase. For this case it seems more appropriate to average the Boltzmann equation over gyrophase which results in the well-known pitch angle evolution equation for the particle transport (e.g. Skilling 1975; Webb 1985; Kirk, Schlickeiser \& Schneider 1988). In the present application energy changes due to radiative (e.g. synchrotron) losses or second-order Fermi acceleration have not yet been considered. The inclusion of radiative losses introduces an upper bound to the possible particle energy when acceleration is balanced by losses and therefore may lead to a cut-off in the momentum spectrum. The effects of second-order Fermi acceleration would lead to an additional diffusion flux in momentum space and may be taken into account by a more careful treatment of the scattering term in the Boltzmann equation (cf. WJM 94; Schlickeiser 1989), which in the current approach (e.g. following Webb 1989) has been modelled by a simple BGK collision term. 


\section{Chapter 4}

\section{Rotating jets in binary black holes}

\subsection{Introduction}

The study of variability in extragalactic objects (e.g. AGN) is usually considered as an important diagnostical tool for the underlying jet physics and the corresponding particle acceleration mechanisms and nonthermal emission processes. The observed time scale $\Delta t_{\mathrm{obs}}$ for short-time variability, for example, allows of an estimate of the (intrinsic) source size $R \sim c \Delta t_{\mathrm{obs}} / 2$, where $c$ is the velocity of light. On the other hand, the observation of periodical variations (on larger time scales) may yield insights into the internal structure of astrophysical jets (e.g. internal rotation: Camenzind \& Krockenberger 1992) or the presence of an external effect causing the observed periodicity (e.g. precession of jets or rotation of jets in a binary system). In order to gain an adequate interpretation of the observed variability one thus has to discriminate between a real jet-intrinsic cause (e.g. internal rotation or effects depending on the acceleration and emission mechanism) and a rather extrinsic cause (e.g. depending on the jet morphology) which (only) mimics the results of the former. Modelling the variability behaviour in AGN may thus be of particular relevance in order to discern the real cause.

In this chapter we consider the noteworthy case of the well-observed blazar Mkn 501. A simple model is presented showing that the TeV and X-ray periodicity of $\sim 23$ days observed during a flaring state in 1997 may basically be interpreted as a doppler-shifted flux modulation due to the (extrinsic) ro-

\footnotetext{
${ }^{1}$ For a source region which moves relativistically, the appropriate relation should be $R \sim c \delta \Delta t_{\text {obs }} / 2(1+z)$, where $\delta$ denotes the doppler factor and $z$ the redshift
} 
tation of a relativistic jet in a binary black hole system. An intrinsic orbital period of $(6-14)$ yrs and a centre-of-mass distance of $(2.0-3.5) \times 10^{16} \mathrm{~cm}$ is derived for the special relativistic jet properties which one may infer from theoretical emission models. If the binary is very close with a separation of an order of which gravitational radiation becomes dominant, an upper limit on the allowed binary masses might be set. Interestingly, the derived values are in accordance with the black hole masses expected from merger scenarios. Hence, the case of Mkn 501 may not only illustrate the influence of an extrinsic cause on the observed variability, but may also strengthen the evidence for the existence of binary black holes in AGN. In the following sections the plausibility of binary systems in AGN is shortly reviewed and later applied to Mkn 501.

\subsubsection{Evolution of binary black hole systems}

Binary black hole systems (BBHSs) are expected to be a common phenomenon in the universe as a result of mergers between galaxies. In the underlying picture for the morphological evolution, galaxies were formed as part of a hierarchical clustering process (e.g. White 1997). Following an old hypothesis by Toomre \& Toomre 1972, giant elliptical galaxies, such as the host galaxy of Mkn 501, might be regarded as products of mergers between spiral galaxies (cf. also Fritze v.- Alvensleben 1996, Kauffmann 1996). Detailed numerical simulations have indeed shown that merging between two spiral galaxies of comparable mass leads to the formation of a spheroidal merger remnant whose physical properties (e.g. density profiles, gravitational radii, surface brightness, mean velocity dispersions) are similar to those observed from elliptical galaxies (e.g. Barnes \& Hernquist 1992). Since the brightest galaxies generally seem to contain massive black holes in their nuclei (e.g. see sect. 1.2), merging would then naturally lead to the formation of massive BBHS (Begelman et al. 1980, abbreviated: BBR 80; Rees 1994; Artymowicz 1998; Richstone 1998; Merritt 1999).

Based on the pioneer work of Begelman, Blandford and Rees 1980 (BBR 80; see also Artymowicz 1998), the evolution of a pair of two black holes with masses of the order of $10^{8} M_{\odot}$, where each is embeded in a dense stellar cluster, could be divided into several stages (see Fig. 4.1): first, due to dynamical friction resulting from the fluctuating part of the gravitational field of a random distribution of stars (cf. Chandrasekhar 1943, Ostriker \& Tremaine 1975), the initial stage is characterized by a rapid approach of the nuclei on a time scale of $t_{\mathrm{df}}<10^{8}$ yrs. The second stage, which occurs on a similar time scale, is marked by the settling of the secondary black hole 
towards the core of the merged system until the two black holes may be considered as a true, gravitationally bound binary with orbital speed exceeding the velocity dispersion of the stars. The subsequent evolution of the binary is then controlled by the formation of a "loss cone" in the stellar distribution around the binary at a separation $d_{\mathrm{lc}}$, i.e. the formation of a phase space region surrounding the binary which contains elongated stellar orbits with ideal impact parameter and which gets depleted due to close encounter with the binary. Repopulation of the loss cone (e.g. by diffusion) occurs on a very long time scale, i.e. the time scale for the binary evolution $t_{h}$ jumps up to $\sim 10^{11}$ yrs. Hence, one typically might expect the binary to spend most of its time at the corresponding separation of $\sim 0.05-1 \mathrm{pc}$. However, this conclusion is only valid as long as the binary does not lose further angular momentum e.g. by slingshot interaction with new stars from subsequent merging events (Roos 1988; Roos et al. 1993), infall of gas (BBR 80; Gould \& Rix 2000) or by interactions with an accretion disk (Ivanov et al. 1999). For, in case of loss, gravitational radiation would eventually become important and the binary evolution could proceed very rapidly to coalescence (i.e. on a time scale $\left.t_{\mathrm{GR}} \propto d^{4}\right)$.

\subsubsection{Evidence for binary black holes}

Up to now, a whole range of phenomena have been attributed to binary black hole systems: Examples include explanations for the observed misalignment (Conway \& Wrobel 1995; see also Appl, Sol \& Vicente 1996), precession (BBR 80) or wiggling of extragalactic jets. The latter phenomenon for example, is supposed to be induced by orbital motion (Kaastra \& Roos 1992; Roos et al. 1993).

Double-peaked Balmer lines of certain broad-line radio galaxies (e.g. 3C 390.3) have been explained as doppler-displaced peaks due to two separate broad line regions, each associated with its own black hole in a supermassive binary at the center of the associated host galaxy (Gaskell 1996a, 1996b; but see also Eracleous et al. 1997).

Periodic outburst activity in the well-observed quasar OJ 287 has commonly been related to a binary system. Several models have been proposed according to which the observed periodicity of about 12 yrs might be related to tidal perturbations (Sillanpää et al. 1988) or to one black hole crossing the accretion disk of the other (Lehto \& Valtonen 1996). On the other hand, Villata et al. (1998) have considered a pair of two bent jets in a binary system, whereas Katz (1997) has suggested the relevance of Newtonian-driven precession when the orbital plane of the binary is inclined to the accretion 


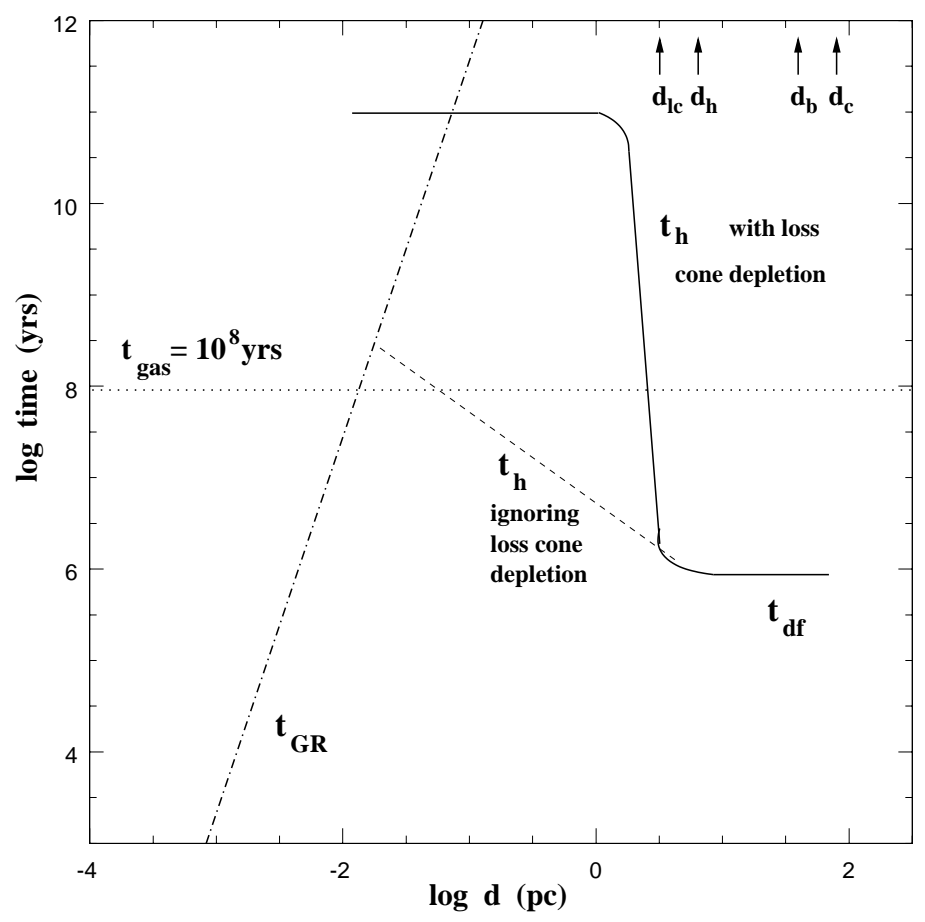

Figure 4.1: Sketch, illustrating the time scales involved in the evolution of a massive binary black hole system with masses $M=10^{8} M_{\odot}$ and $m=0.3 M$ and separation $d$. The binary is assumed to lose angular momentum by gas accretion onto the larger black hole which leads to an evolution time scale $t_{\text {gas }} \simeq 10^{8}$ yrs (after Begelman et al. 1980). The merger remnant has been modelled as an elliptical galaxy with core radius $d_{c}$. At the separation $d_{b}$ the holes may be considered as bound to each other, while the binary becomes hard at $d_{h}$ where the orbital speed exceeds the ambient velocity dispersion.

disk.

Precession of the (inner) jet in a binary system has also been proposed as an explanation for the observed kinematic behaviour of superluminal features (velocities, position angles) in the quasar 3C273 (e.g. Abraham \& Carrara 1998; Abraham \& Romero 1999). There is also mounting observational evidence for a helical motion of jet components in other extragalactic radio sources. A three-year VLBI monitoring of the blazar PKS 0420-014, for example, has recently revealed superluminal jet components following curved trajectories, suggested to be explainable within the framework of a binary black hole system (Britzen et al. 2000).

Additional support for the interacting and merging scheme may also been found in the observations of radio sources with double nuclei (e.g. 3C75: 
Owen 1985). There are about $10^{4}$ known quasars of which about 40 are pairs or multiples with separation smaller than $10^{\prime \prime}$ (e.g. Kochanek et al. 1999). Most of them seem to be confirmed gravitational lenses, but there is increasing evidence for true binaries (where no plausible lens candidate has been found and the optical and radio flux ratios are highly discrepant). With the recent detection of the binary quasar associated with FIRST J164311.3+315618 (Brotherton 1999), which is the smallest separation binary quasar so far identified (separation $2 .{ }^{\prime \prime} 3, z=0.586$ ), at least four binary quasars seem to be confirmed. As has been noted by Mortlock et al. (2000), one should observe about one percent of all merger-formed quasars to be in a double nucleus system, if one is taking into account that only collision between two large galaxies can result in a binary quasar and is estimating the result using a Schechter mass function. A recent Hubble Space Survey of $\sim 100$ BL Lac objects with redshift $0.05 \leq z \leq 1.2$ also shows three BL Lac objects with double nuclei (with a projected distance on the kpc-scale). However, it has not been clarified so far whether they are true binaries or gravitational lensed objects (Scarpa et al. 1999).

\subsection{The gamma-ray blazar Mkn 501}

In the particular case of Mkn 501, the complex morphology of its radio jet and the peculiar behaviour of its spectral energy distribution (SED) have prompted elaborate models relating these properties to a binary black hole system: Conway \& Wrobel (1995), for example, have proposed a saturated helix model in order to explain the misalignment of the radio jet on parsec and kiloparsec scales. Recently, Villata \& Raiteri (1999) have argued that the X-ray variations in the SED of Mkn 501 might be due solely to the changing orientation of a helical synchrotron emitting jet in a close black hole binary. In the following, we show that the recent discovery of periodicity in the $\mathrm{TeV}$ and X-ray fluxes of Mkn 501, believed to be associated with moving features in the jet of Mkn 501, might add another aspect for assessing the relevance of a binary black hole system in this galaxy.

Mkn 501 is one of at least four active galactic nuclei which have been detected at $\mathrm{TeV}$ energies (for review, see Catanese \& Weekes 1999). Being the second closest among these with a redshift of $z=0.034$, Mkn 501 has in the past been classified as an X-ray selected BL Lac object showing virtually no emission lines. Its host galaxy is known to be the elliptical galaxy UGC 10599 (Stickel et al. 1993). As a BL Lac object, Mkn 501 belongs to the blazar class of AGN which are thought to have relativistic jets oriented at a small viewing 
angle. The flux measured by a distant observer thus appears to be enhanced by Doppler boosting (e.g. see sect. 1.3).

At the beginning of 1997, Mkn 501 had suddenly undergone a phase of high activity becoming the brightest source in the sky at TeV energies. Subsequent multiwavelength campaigns revealed a variable, two component SED with a low energy part extending up to $100 \mathrm{keV}$ (Pian et al. 1998) and a high energy part which extends at least up to $20 \mathrm{TeV}$ (Samuelson et al. 1998; Konopelko 1999; for implications, see for example Mannheim 1998). During this activity phase, particular types of variability have been observed (e.g. Protheroe et al. 1998), consisting of flaring episodes of several days and additional intraday-variabilities. While the $\mathrm{TeV}$ and $\mathrm{X}$-rays variations seem to be well correlated, the evidence for correlations with the optical U-band appears to be rather weak (e.g. Catanese et al 1997; Djannati-Atai et al. 1999; Aharonian et al. 1999, Petry et al. 2000). One of the most fascinating features is the observed periodicity in the $\mathrm{TeV}$ region with a period in the range of $(23-26)$ days, which has been found in the data taken by several Cherenkov telescopes (see Protheroe et al. 1998; Hayashida et al. 1998). For illustration Fig. 4.2 shows the lightcurve of Mkn 501 above $1.5 \mathrm{TeV}$ as measured by the stereoscopic HEGRA system, an array consisting of six air Cherenkov telecopes located on the Canary Island of La Palma (Spain). A recently performed fourier analysis of the X-ray data from April to July 1997 taken by the all sky monitor (ASM) onboard the Rossi X-ray timing explorer (RXTE) (e.g. Fig. 4.3) additionally seems to support a periodicity of $\sim 23$ days (Kranich et al. 1999; Nishikawa et al. 1999), see Fig 4.4.

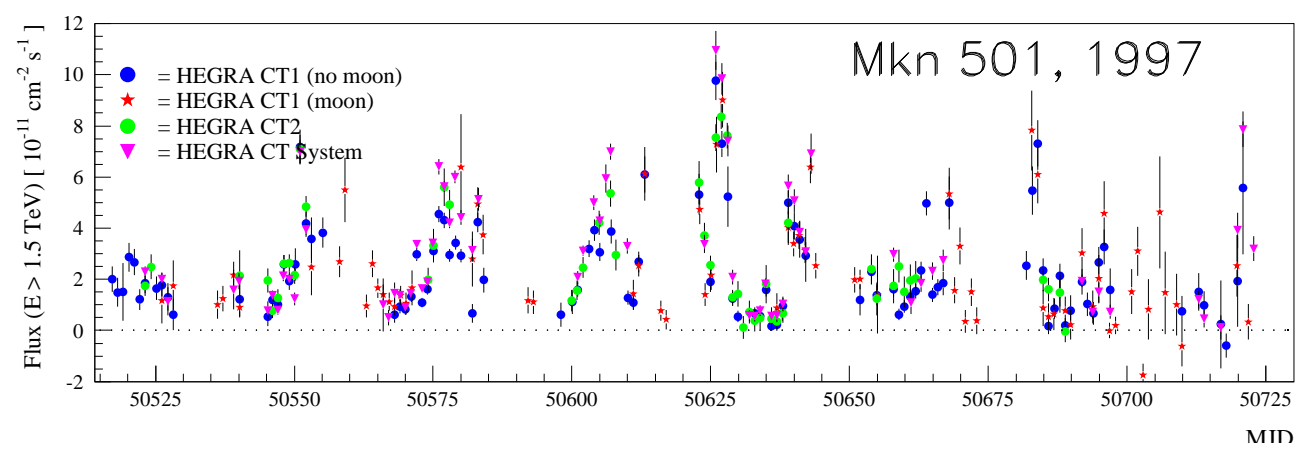

Figure 4.2: Observed flux variability of Mkn 501 above $1.5 \mathrm{TeV}$ as measured by the HEGRA Cherenkov telescope system in 1997 (MJD [24]50540= April 1, 1997). Figure adopted from Kranich et al. 1999.

In the following section, we consider the possibility that this periodicity in the flaring state arises due to the orbital motion of the relativistic jet in a binary 


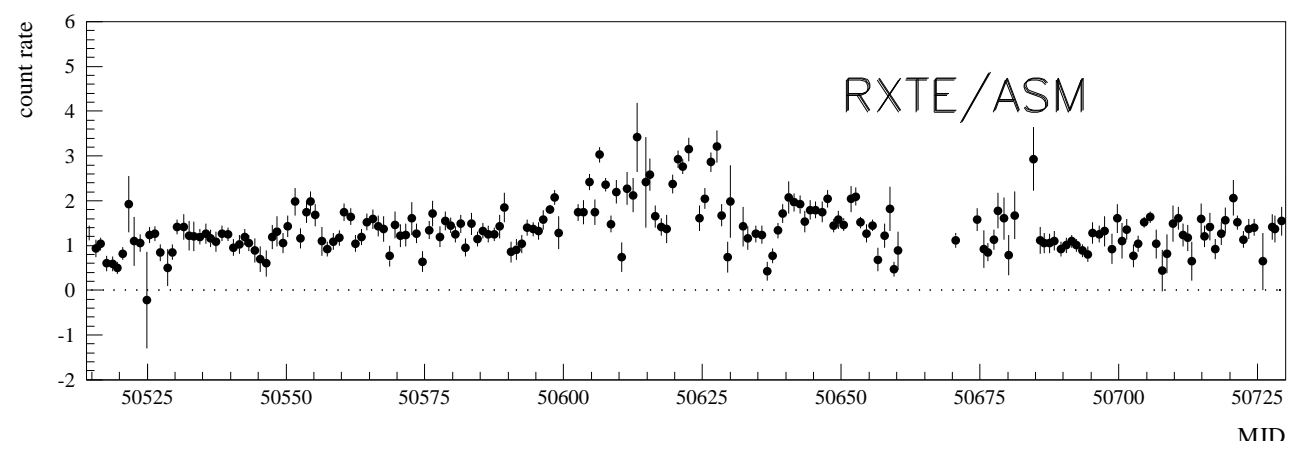

Figure 4.3: Observed X-ray flux variability of Mkn 501 as measured by RXTE between $2-10 \mathrm{keV}$. The data are binned in daily bins for clarity. Figure adopted from Kranich et al. 1999.

black hole system. The nonthermal radiation is supposed to be emitted by a relativistic jet which emerges from the less massive black hole and the periodicity thus being due mainly to geometrical origin (i.e. Doppler-shifted modulation).

\subsection{Possible evidence for a binary system in Mkn 501}

\subsubsection{Doppler-shifted flux modulation}

Consider a simple binary model for Mkn 501 where the binary orbit is assumed to be circular, because dynamical friction between two parent galaxies during the merger might ensure that the initial eccentricity of the resulting binary is small (e.g. Polnarev \& Rees 1994). Using Kepler's third law, the intrinsic orbital frequency $\Omega_{k}$ of a binary with separation $d$ is given by

$$
\Omega_{k}=\frac{\sqrt{G(m+M)}}{d^{3 / 2}}
$$

where $m$ and $M$ denotes the mass of the smaller and the larger hole respectively, and $G$ is the gravitational constant.

Let us further assume the observed jet to be formed by the less massive black hole and the nonthermal X-ray and $\gamma$-ray radiation in the flaring state being emitted by a relativistic emission region (e.g. knot, blob or shock) which propagates outwards from the core along the jet with gamma factor $\gamma_{b}$. Owing to the (non-relativistic) orbital motion, the true trajectory of 


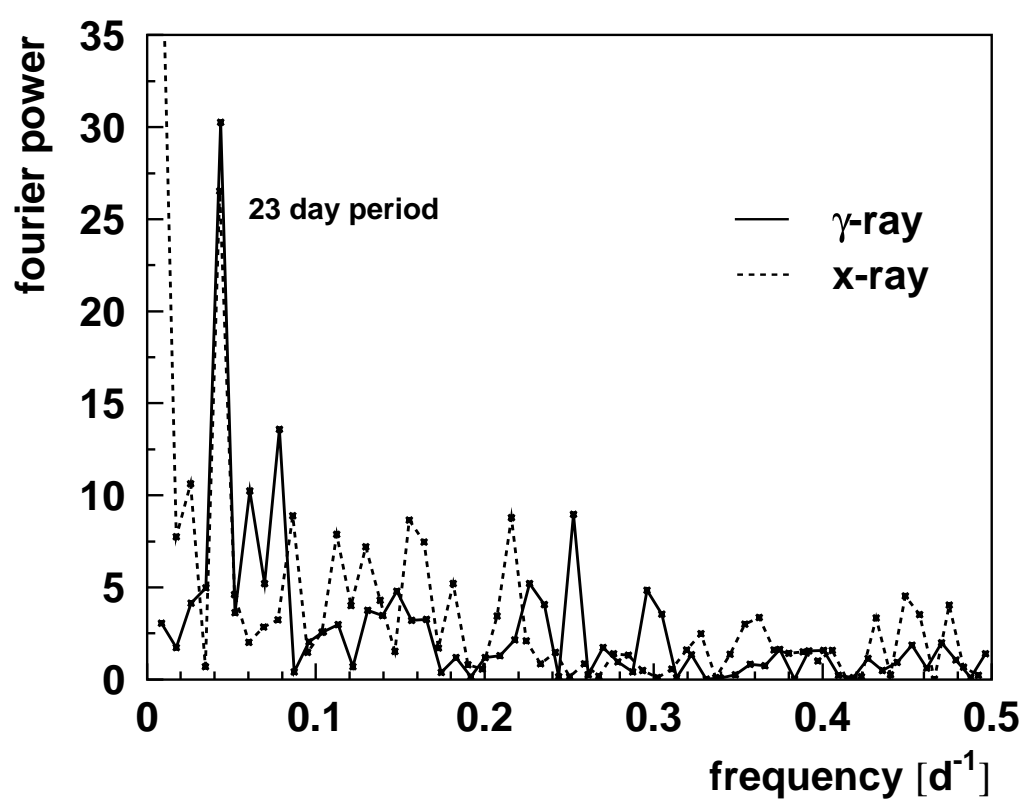

Figure 4.4: Fourier analysis of the $\mathrm{TeV}$ and $\mathrm{X}$-ray data using the normalized Lomb periodogram. The periodicity of $\simeq 23$ days is clearly identifiable. Figure courtesy of D. Kranich.

the knot is a long stretched helix (see Fig. 4.5). The modulation of the emission then occurs as a consequence of the slight variation of the inclination angle due to the orbital $\phi$-component of the velocity field of the knot. This observed flux modulation by Doppler boosting is well-known (e.g. Blandford \& Königl 1979; Rieger 1997). For a resolved emission region (e.g. a blob of plasma) with spectral index $\alpha$, the spectral flux modulation by doppler boosting can be written in the form (see sect. 1.3)

$$
S(\nu)=\delta^{3+\alpha} S^{\prime}(\nu),
$$

where $S^{\prime}$ is the spectral flux density measured in the comoving frame and $\delta$ denotes the Doppler factor given by

$$
\delta(t)=\frac{1}{\gamma_{b}\left[1-\beta_{b} \cos \theta(t)\right]},
$$

with $\theta(t)$ as the actual angle between the velocity $\vec{\beta}_{b}=\dot{\vec{x}}_{b}(t) / c$ of the emission region and the direction of the observer. The bulk Lorentz factor is defined by $\gamma_{b}=\left(1-\beta_{b}^{2}\right)^{-0.5}$.

Due to the orbital motion around the center-of-mass, the Doppler factor for the emission region is a periodical function of time. In the simplest 


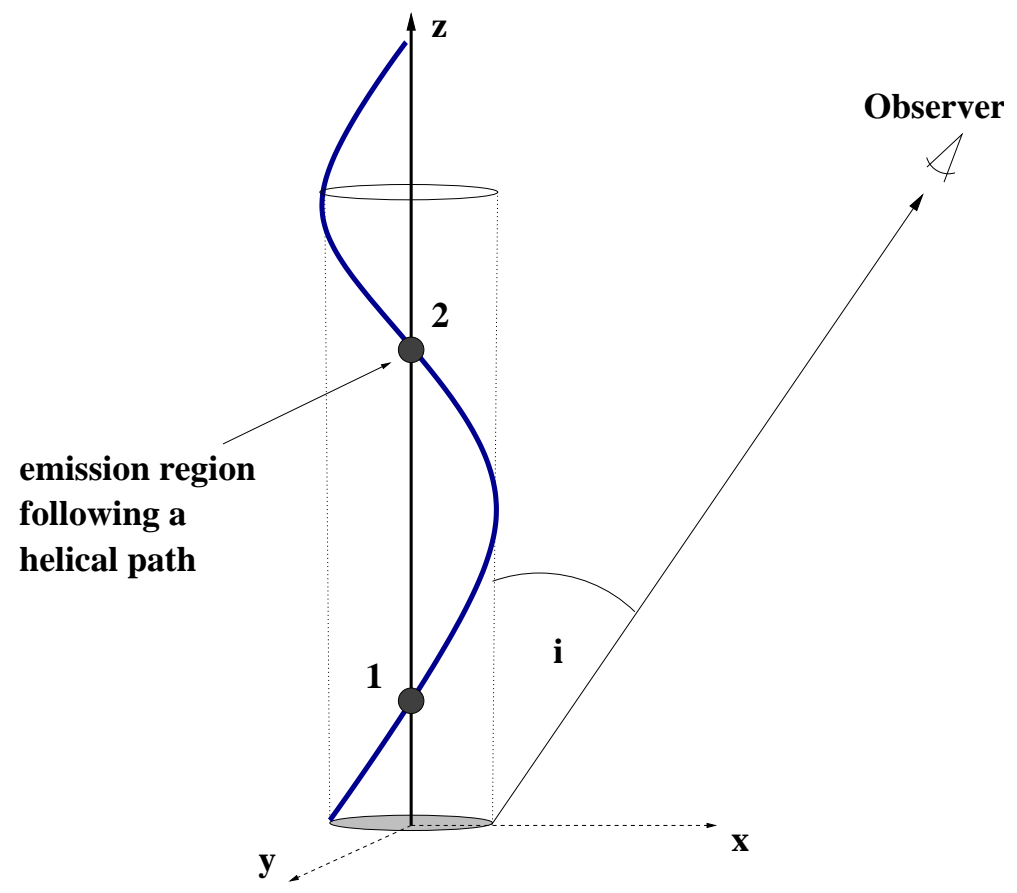

Figure 4.5: Idealized sketch of the assumed model, where an emission region follows a helical jet path. Due to differential doppler boosting the flux measured by an observer (lying in the $(x, z)$-plane with inclination $i$ to the central axis) from position 1 appears enhanced relative to the flux observed from position 2 .

case where the angle between the jet axis and the direction of the total angular momentum of the binary is assumed to be zero (e.g. neglecting any kind of precessional motion) the Doppler factor may be deduced using the following arguments: Denote $\vec{i}=(\sin i, 0, \cos i)$ as the normalized vector directing towards the observer and let $\vec{x}_{b}(t)=\left(R \cos \Omega_{k} t, R \sin \Omega_{k} t, v_{z} t\right)$ be the position vector of the emission region, with $R=M d /(m+M)$ the centre-of-mass distance and $v_{z}$ the outflow velocity in the direction of the total angular momentum. Then, noting that $\cos \theta(t)=\vec{i} \times \dot{\vec{x}}_{b}(t) /\left(|\vec{i}|\left|\dot{\vec{x}}_{b}(t)\right|\right)$, the Doppler factor Eq. (4.3) may be written as

$$
\delta(t)=\frac{\sqrt{1-\left(v_{z}^{2}+\Omega_{k}^{2} R^{2}\right) / c^{2}}}{1-\left(v_{z} \cos i-\Omega_{k} R \sin i \sin \Omega_{k} t\right) / c},
$$

with $c$ the velocity of light. Obviously, the Doppler factor Eq. (4.4) becomes maximal for $t=0.75 P_{k}$ and minimal for $t=0.25 P_{k}$, where $P_{k}=2 \pi / \Omega_{k}$ denotes the keplerian period. The effect of doppler boosting is illustrated for appropriate values in Fig. 4.6. From the TeV flux ratio of $f \sim 8$ between 


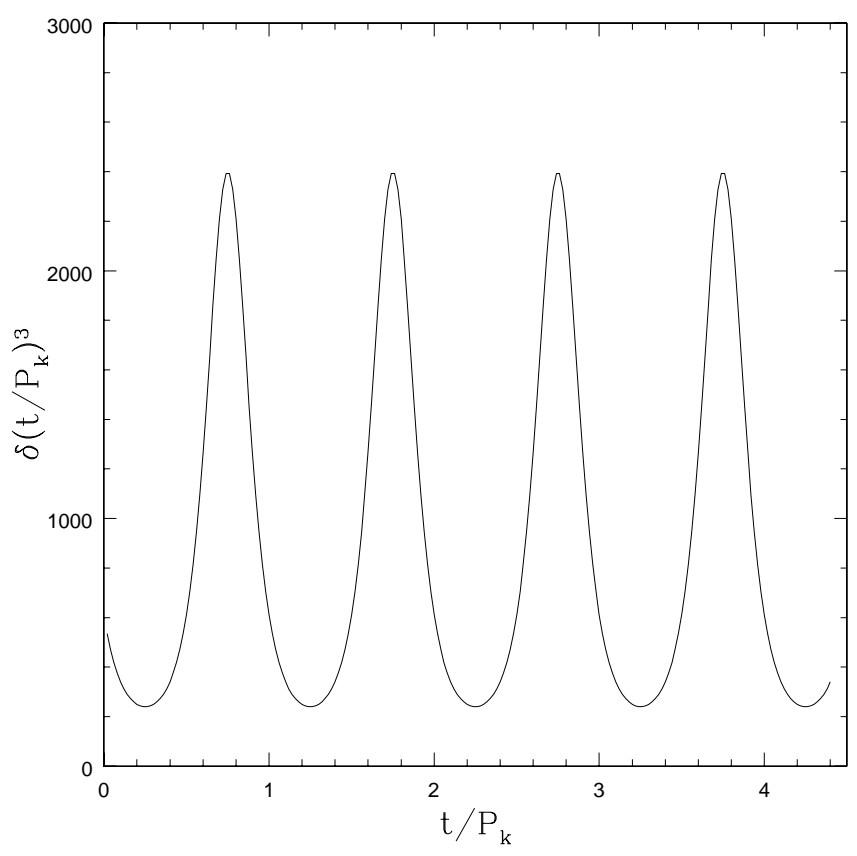

Figure 4.6: Flux modulation assuming only differential doppler boosting. The $y$ co-ordinate gives the relative intensity $\delta(t)^{3}$ as a function of time.

the maximum and the minimum state during the observation (cf. Protheroe et al. 1998; Hayashida et al. 1998, Aharonian et al. 1999) and the assumption that the periodicity arises mainly due to geometrical origin, we now obtain the condition $\delta_{\max } / \delta_{\min } \simeq f^{1 /(3+\alpha)}$ [see Eq. (4.2)]. Consequently, by using Eq. (4.4) one finds

$$
\Omega_{k} R=\frac{f^{1 /(3+\alpha)}-1}{f^{1 /(3+\alpha)}+1}\left(\frac{1}{\sin i}-\frac{v_{z}}{c} \cot i\right) c .
$$

For a source region which moves in the time interval $d t$ from point $A$ to point $B$ with relativistic velocity $v_{z}$ and at an angle $\psi$ to the line of sight, the observed difference in arrival times for radiation emitted at $A$ and $B$ is generally given by $d t_{\mathrm{obs}}=d t-d t\left(v_{z} / c\right) \cos \psi$, thus leading to a shortening of the observed time interval. Along this line of argument, one may easily derive that the observer in the model presented here will only perceive a strongly shortened period, i.e. the observed period $P_{\text {obs }}$ is related to the intrinsic period $P_{k}$, measured in the frame fixed in the center of the galaxy, by (cf. Camenzind \& Krockenberger 1992, Roland et al. 1994)

$$
P_{\text {obs }}=(1+z) \int_{0}^{P_{k}}\left(1-\beta_{b} \cos \theta(t)\right) \mathrm{d} t .
$$


Performing the integration, one immediately arrives at

$$
P_{\mathrm{obs}}=(1+z)\left(1-\frac{v_{z}}{c} \cos i\right) P_{k}
$$

From a theoretical point of view, relativistic blazar jets are thought to be oriented at a small viewing angle. Current emission models favour an inclination angle $i \simeq 1 / \gamma_{b}$ (Spada 1999; cf. also Chiaberge et al. 2000) with typical bulk Lorentz factors in the range 10-15 (e.g. Mannheim et al. 1996; Hillas 1999; Spada et al. 1999). For such values and by using an observed period of 23 days and a characteristic outflow velocity of $v_{z} / c \simeq\left(1-1 / \gamma_{b}^{2}\right)^{0.5}$, Eq. (4.7) results in an intrinsic period of

$$
P_{k}=(6-14) \text { yrs }
$$

Combining Eq. (4.7) and Eq. (4.5) we may also derive an expression for the centre-of-mass distance

$$
R=\frac{P_{\mathrm{obs}}}{2 \pi(1+z)} \frac{f^{1 /(3+\alpha)}-1}{f^{1 /(3+\alpha)}+1} \frac{c}{\sin i} .
$$

Given the observed period and the spectral index, Eq. (4.9) only depends on the inclination angle. Accordingly, for an observed period of 23 days, a ratio $f=8$ and a $\mathrm{TeV}$ spectral index of $\alpha \simeq(1.2-1.7)$ (cf. Aharonian et al. 1999), one gets

$$
R \simeq(2.0-3.5) \times 10^{16} \mathrm{~cm},
$$

using the inclination values above.

By inserting Eq. (4.1) in Eq. (4.9), noting that $R=M d /(m+M)$, the appropriate binary mass ratio is given by

$$
\begin{aligned}
\frac{M}{(m+M)^{2 / 3}}= & \frac{P_{\mathrm{obs}}^{1 / 3}}{(2 \pi[1+z] G)^{1 / 3}} \frac{c}{\sin i} \\
& \times \frac{f^{1 /(3+\alpha)}-1}{f^{1 /(3+\alpha)}+1}\left(1-\frac{v_{z}}{c} \cos i\right)^{2 / 3} .
\end{aligned}
$$

For a secondary mass in the range of $\left(10^{6}-10^{8}\right) M_{\odot}$ the required primary masses are shown in Fig. 4.7 [i.e. the curves $K(10), K(15)$ ] for two different inclination angles and $f=8$, yielding primary masses of the order of $10^{8} M_{\odot}$. 


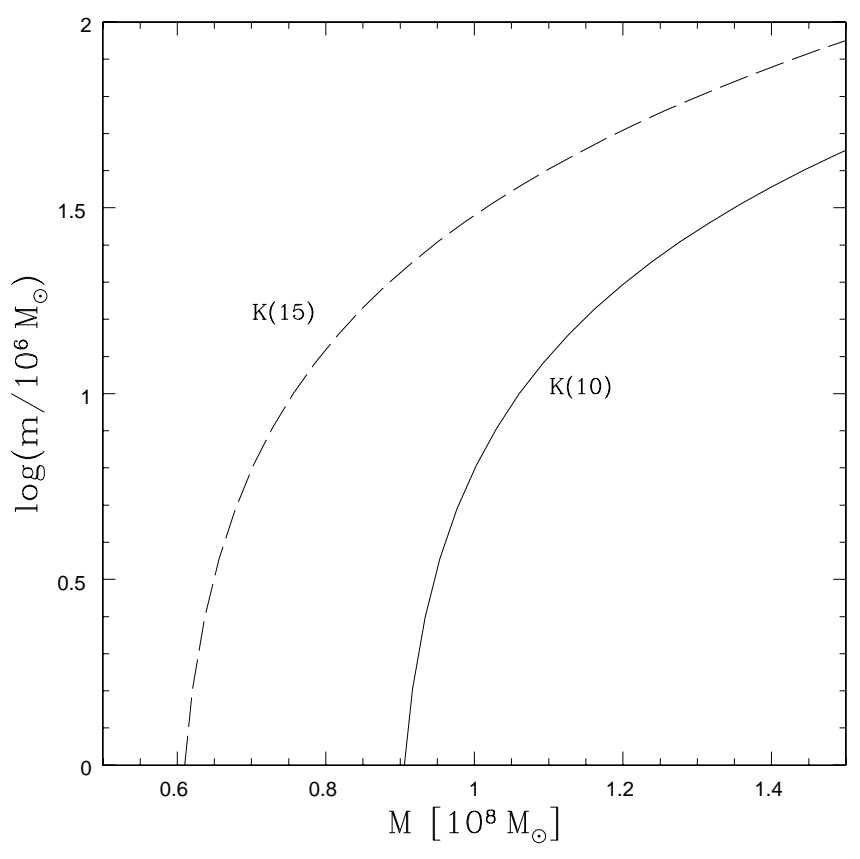

Figure 4.7: Required mass dependence $K(1 / i)$ for a binary black hole system in Mkn 501. The solid $[K(10)]$ and long dashed $[K(15)]$ curve are given by the Doppler condition Eq. (4.11) for inclination angles $i=1 / \gamma_{b}$ with $\gamma_{b}=10$ and 15, respectively. A TeV spectral index of 1.2 and an observed period of 23 days have been applied for the calculation.

\subsubsection{Upper limits for the binary masses}

Observationally, BL Lacs are generally less luminous radio sources, showing a lack of strong optical emission lines and rather little signs of cosmological evolution (cf. Bade et al. 1998; Cavaliere \& Malquori 1999). Celotti et al. (1998) have suggested that BL Lac objects correspond to the final evolutionary stage of sources accreting at low radiative efficiencies (i.e. a dormant black hole system). This seems to be supported by HST observation, indicating that the less luminous AGN stages occur after the original quasar has dimmed (Bahcall et al. 1994). Recently, Villata \& Raiteri (1999) have argued that BL Lacs represent advanced and close binary black hole systems with a decreased mass accretion rate, the binary separation in the case of Mkn 501 being of an order for which gravitational radiation becomes dominant. As outlined in sect. 4.1.1, the formation of a loss cone in the stellar distribution around the binary and a decreasing mass accretion rate would generally result in a hardening of the binary above the separation at which gravitational 
radiation eventually becomes dominant. This state may thus be associated with little signs of cosmological evolution and a less luminous stage. Therefore, one might set an upper limit on the allowed binary masses in Mkn 501 by assuming that the current separation equals the gravitational separation $d_{g}$, i.e. the position where the gas dynamical time scale is balanced by the time scale for gravitational energy losses (BBR 80). Accordingly, let us suppose that gas has been constantly supplied up to now (for example, by tidal interaction between galaxies, cf. Heidt 1999) and accreted onto the more massive black hole. Theoretical support for such a picture may be found in simulations of merging galaxies (e.g. Barnes \& Hernquist 1996) indicating that interaction drives gas towards the central regions of the galaxy where it can be used as fuel for new activity. In such a case the binary separation shrinks on a time scale of

$$
t_{\mathrm{gas}} \simeq M \frac{1 M_{\odot} \mathrm{yr}^{-1}}{\dot{M}} \mathrm{yrs},
$$

where $\dot{M}$ denotes the accretion rate (BBR 80$)$. For a simple estimate one may assume that during the optical bright QSO phase mass accretion occurs at about the Eddington limit. Since this phase of nuclear activity seems to be rather short with a typical duration of a few times $10^{7}$ yrs (Haehnelt et al. 1998; Richstone et al. 1998), the gas dynamical time scale should be small also, i.e. the gas accretion rate should be of the order of one solar mass per year for the primary masses expected from the results of sect. 4.3.1. In particular, for a duration of nuclear activity of the order of the salpeter time

$$
t_{\mathrm{s}}=\eta \sigma_{T} c / 4 \pi G m_{p}=4.5 \eta_{0.1} \times 10^{7} \mathrm{yrs},
$$

and for a primary black hole mass of $\sim 10^{8} M_{\odot}$, gas infall rates of $\sim 2 M_{\odot} \mathrm{yr}^{-1}$ would have been required in order to sustain the Eddington luminosity, using a canonical 10\% efficiency. For such values, the gas dynamical time scale and the salpeter time scale are about the same size.

On the other hand, assuming a circular orbit, the decrease of the total energy $E=-G M m /(2 d)=-G \mu M_{\text {tot }} /(2 d)$ (cf. virial theorem) via gravitational radiation may be written as (e.g. Misner, Thorne \& Wheeler 1973)

$$
\frac{\mathrm{d} E}{\mathrm{~d} t}=-\frac{32}{5} \frac{G^{4}}{c^{5}} \frac{\mu^{2}}{M_{\mathrm{tot}}^{3}} d^{5},
$$

where the term on the right hand side denotes the power radiated in gravitational waves, $M_{\text {tot }}=m+M$ is the total mass and $\mu=m M /(m+M)$ the reduced mass. Using Eq. (4.14) the time scale $\tau_{\text {grav }}=d / \dot{d}$, on which 
gravitational radiation shrinks the orbit, then is given by

$$
\tau_{\text {grav }}=\frac{5}{64} \frac{c^{5}}{G^{3}} \frac{1}{M m(m+M)} d^{4} .
$$

By applying appropriate values, Eq. (4.15) may be written as

$$
\tau_{\text {grav }}=2.52 \times 10^{5} \frac{d_{16}^{4}}{M_{8} m_{8}\left(m_{8}+M_{8}\right)} \mathrm{yrs},
$$

where the distance and the masses are expressed in units of $10^{16} \mathrm{~cm}$ and $10^{8} M_{\odot}$, respectively.

Solving Eq. (4.14) for the separation $d$, the solution is simply given by $d(t)=$ $d_{0}\left(1-a t / d_{0}^{4}\right)^{0.25}$, where $d_{0}=d(t=0)$ and $a=256 G^{3} \mu M_{\mathrm{tot}}^{2} /\left(5 c^{5}\right)$. Thus, for the assumed separation, the expected binary gravitational lifetime $\tau_{\text {life }}$ may then be defined as the spiral time in which the binary black holes will spiral together (i.e. $\tau_{\text {life }}=d_{0}^{4} / a=\tau_{\text {grav }} / 4$ with $d_{0}=d_{g}$ the assumed binary separation).

Now, by equating the gas dynamical time scale $t_{\text {gas }}$ with $\tau_{\text {grav }}$, the separation at which gravitational radiation becomes dominant may be written as

$$
d_{g}=4.46 \times 10^{16} M_{8}^{1 / 2} m_{8}^{1 / 4}\left(m_{8}+M_{8}\right)^{1 / 4}\left(\frac{M_{\odot} \mathrm{yr}^{-1}}{\dot{M}}\right)^{1 / 4} \mathrm{~cm} .
$$

Using Eq. (4.17) and Eq. (4.1) we immediately arrive at the relation

$$
\begin{aligned}
\frac{M^{1 / 2} m^{1 / 4}}{(m+M)^{1 / 12}}= & 1.82 \times 10^{22}\left(\frac{\dot{M}}{M_{\odot} \mathrm{yr}^{-1}}\right)^{1 / 4} \\
& \times \frac{P_{\mathrm{obs}}^{2 / 3}}{(2 \pi[1+z])^{2 / 3}\left(1-\frac{v_{z}}{c} \cos i\right)^{2 / 3}}[\mathrm{~g} / \mathrm{s}]^{2 / 3} .
\end{aligned}
$$

This mass dependence is illustrated in Fig. 4.8 (curves $G$ ). The respective upper limit is given by the point of intersection with the relevant curve $K$. For example, applying $\alpha=1.2$ and using $i=1 / 10$, we have a maximum secondary mass $m \simeq 7 \times 10^{6} M_{\odot}$ and a corresponding primary mass of $M \simeq$ $10^{8} M_{\odot}$ for $\dot{M}=1 M_{\odot} /$ yr (cf. also Table 4.1). The masses shown in Fig. 4.8 are in a reasonable range for ellipticals. Masses of the order of one million solar masses for the companion black hole appear to be in agreement with the concept that the galaxy swallowed in the merger process was a minor spiral galaxy. On the other hand, the host galaxy of Mkn 501 seems to be particularly large and high-luminous (Nilsson et al. 1999) and therefore probably belongs to those classes of ellipticals which may have black holes in the centers of at least a few hundred million solar masses (cf. sect. 1.2). A binary scenario for Mkn 501 may properly account for such a difference. 


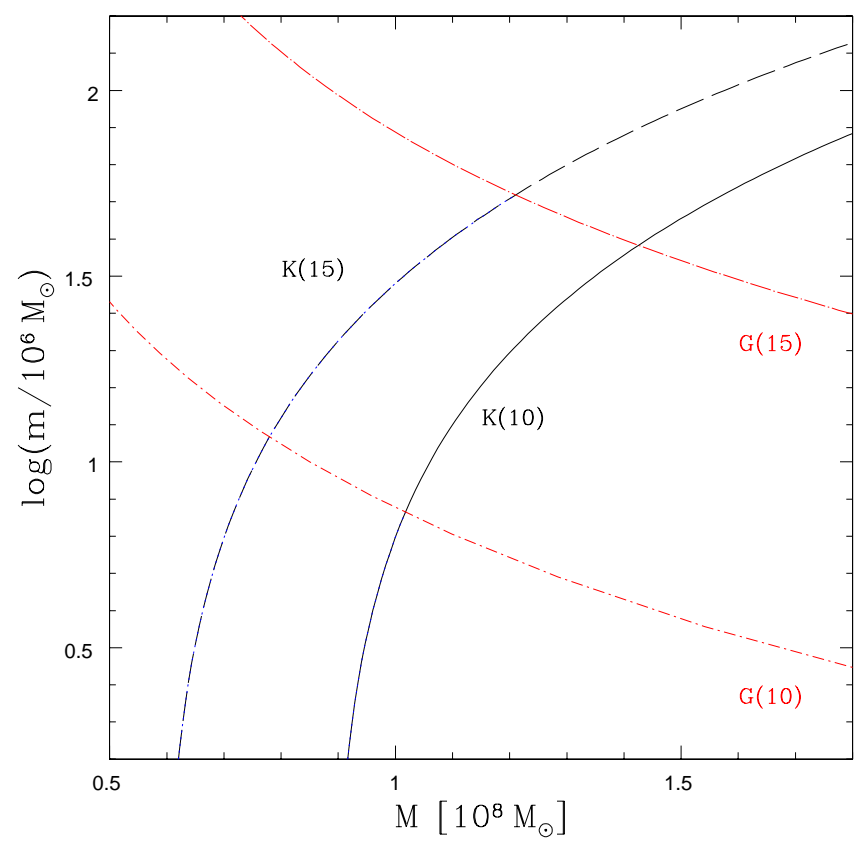

Figure 4.8: Required mass dependence for a binary black hole system in Mkn 501 (see also Fig. 4.7). The solid $[K(10)]$ and long dashed $[K(15)]$ curve are given by the Doppler condition Eq. (4.11) for inclination angles $i=1 / \gamma_{b}$ with $\gamma_{b}=10$ and 15 , respectively. The curves $G(1 / i)$ are given by the condition that the current binary separation equals the gravitational distance $d_{g}$, see Eq. (4.18), and may thus be considered as upper limits on the allowed binary masses. The allowed part for the masses has been drawn in blue. A TeV spectral index of 1.2 , an inflow rate $\dot{M}=1 M_{\odot} /$ yr and an observed period of 23 days have been applied for the calculation.

\subsection{Discussion}

In this chapter we have suggested that the periodicity in the flaring state observed in Mkn 501 might be caused by the orbital motion of the jet in a close binary black hole system. The periodical behaviour may thus be primarily considered as the result of an extrinsic cause. In applying a simple toy-model it has been shown that the binary system may have a period of $\sim(6-14)$ yrs and a centre-of-mass distance of $\sim(2.0-3.5) \times 10^{16} \mathrm{~cm}$. Several upper limits for binary black hole masses could be derived if one assumes that the current separation roughly corresponds to the distance at which gravitational radiation becomes important. This has been done in Tables 4.1, 4.2 using an observed period of $P_{\text {obs }}=23$ days for mass inflow 
rates $\dot{M}=2 M_{\odot} /$ yr (Tab. 4.1) and $\dot{M}=1 M_{\odot} /$ yr (Tab. 4.2).

The allowed mass ranges interestingly agree with expectations from merger scenarios and recent suggestions for a binary system in Mkn 501 made by Villata \& Raiteri (1999). A direct determination for the mass of the central

\begin{tabular}{|l|ll|ll|}
\hline$i=1 / \gamma_{b}$ & $1 / 10$ & & $1 / 15$ & \\
\hline \hline$m\left[10^{8} M_{\odot}\right]$ & 0.13 & $(0.18)$ & 0.81 & $(0.98)$ \\
\hline$M\left[10^{8} M_{\odot}\right]$ & 1.11 & $(0.92)$ & 1.44 & $(1.31)$ \\
\hline$d\left[10^{16} \mathrm{~cm}\right]$ & 2.58 & $(2.48)$ & 5.40 & $(5.43)$ \\
\hline$P_{k}[\mathrm{yrs}]$ & 6.10 & & 13.7 & \\
\hline$\tau_{\text {life }}\left[10^{7} \mathrm{yrs}\right]$ & 1.57 & $(1.30)$ & 2.04 & $(1.86)$ \\
\hline$P_{p}\left[10^{4} \mathrm{yrs}\right]$ & 0.69 & $(0.59)$ & 5.88 & $(6.05)$ \\
\hline
\end{tabular}

Table 4.1: Maximum allowed binary masses, binary separation $d$, intrinsic orbital period $P_{k}$, gravitational lifetime $\tau_{\text {life }}$ and precessional period $P_{p}$ for inclination angles $i$, accretion rate $\dot{M}=2 M_{\odot} / \mathrm{yr}$, i.e. $t_{\text {gas }} \sim t_{\mathrm{s}}$, and spectral index $\alpha=1.2(1.7)$.

object in spiral galaxies, has been done so far only in a few cases (e.g. M 31, and our own galaxy). The results, however, indicate central masses which do not exceed $10^{7} M_{\odot}$. A recent estimate from the innermost $(<100 \mathrm{pc})$ kinematics of spirals yields upper limits on the black hole masses resident in late-type spirals of $M \leq 10^{6}-10^{7} M_{\odot}$ while the upper limits on early-type (Sa) spirals are in the same range as those for ellipticals (Salucci et al. 2000). This is quite compatible with our derived mass estimates.

Additionally, a recent estimate of the central black hole masses for gammaloud blazars, using luminosity constraints and variability time scales, results in an expected black hole mass for Mkn 501 of $10^{7} M_{\odot}$ (Fan et al. 1999). This result however has been derived by using a small Doppler factor of the order of one and a doubling time scale of $6 \mathrm{~h}$. Nevertheless, their mass estimate [see, for example, their Eq. (4)] remains quite similar, i.e. $m \leq 0.9 \times 10^{7} M_{\odot}$, if one uses a Doppler factor of $\sim 15$ and considers a time scale for variability of less than $20 \mathrm{~min}$, in accordance with recent measurements of optical microvariability in Mkn 501 (Ghosh et al. 2000). The very latest RXTE-results concerning X-variability of Mkn 501 in 1998 on a time scale of less than 10 min (Catanese \& Sambruna 2000) may provide further evidence for (high) doppler boosting.

The TeV observations of Mkn 501 indicate that we may have $N \leq 6$ for the number $N$ of periodic oscillations (cf. Aharonian et al. 1999; Catanese 


\begin{tabular}{|l|ll|ll|}
\hline$i=1 / \gamma_{b}$ & $1 / 10$ & & $1 / 15$ & \\
\hline \hline$m\left[10^{8} M_{\odot}\right]$ & 0.07 & $(0.11)$ & 0.52 & $(0.65)$ \\
\hline$M\left[10^{8} M_{\odot}\right]$ & 1.02 & $(0.82)$ & 1.21 & $(1.09)$ \\
\hline$d\left[10^{16} \mathrm{~cm}\right]$ & 2.47 & $(2.34)$ & 4.95 & $(4.95)$ \\
\hline$P_{k}[\mathrm{yrs}]$ & 6.10 & & 13.7 & \\
\hline$\tau_{\text {life }}\left[10^{7} \mathrm{yrs}\right]$ & 2.89 & $(2.32)$ & 3.44 & $(3.09)$ \\
\hline$P_{p}\left[10^{4} \mathrm{yrs}\right]$ & 0.58 & $(0.47)$ & 4.17 & $(4.17)$ \\
\hline
\end{tabular}

Table 4.2: The same quantities as in Tab. 4.1, but now using an accretion rate $\dot{M}=1 M_{\odot} /$ yr, i.e. $t_{\text {gas }} \sim 2 t_{\mathrm{s}} \sim 10^{8}$ yrs.

\& Weekes 1999; Quinn et al. 1999), which results in a required propagation length for the emitting component of

$$
l_{z}=N P_{k} v_{z} \simeq 11-26 \mathrm{pc} .
$$

Thus, for the projected length at the position of Mkn 501 one finds $l_{p} \simeq 1.4-$ 2.1 mas for the respective angles $i=(1 / 15)-(1 / 10)$ rad. Remarkably, the jet of Mkn 501 bends dramatically at about 3 mas from the core (Marscher 1999; see Fig. 4.9). Hence, a change in the jet parameters might be the reason for the termination of the observed periodicity.

For the proposed model to be valid, the jet has to be perfectly collimated with an intrinsic opening angle of less then $\arctan \left(d / l_{z}\right) \sim 0.05^{\circ}$. Such values are indeed expected in scenarios for the formation and collimation of magnetized BL Lac jets (cf. Camenzind \& Krockenberger 1992; Appl \& Camenzind 1993; Schramm et al. 1993). At first sight however, such a cylindrical jet structure seems to be at least $\sim 20$ times more collimated than the radio jet seen on VLBA maps (cf. Marscher 1999). There is evidence though, for an at least two-component jet structure in Mkn 501 suggesting an inner spine with a transverse magnetic field and an envelope with a longitudinal magnetic field (Aaron 1999; Marscher 1999). The polarization properties of the inner spine strongly support shocked-jet models (cf. Attridge et al. 1999). The proposed model requires in fact that the high energy emission originates in a channel along the jet axis as in two-fluid models (e.g. Sol et al. 1989, Roland et al. 1994), the inner emission probably being self-absorbed on the VLBA scale. Recent observations of radio jets indeed indicate a confinement of the higher energy emission to a well-defined channel within a much more extended radio emission (Bahcall et al. 1995; Perlman et al. 1999; Swain et al. 1998) giving credit to a central spine-shear layer morphology (cf. also Giovannini et 


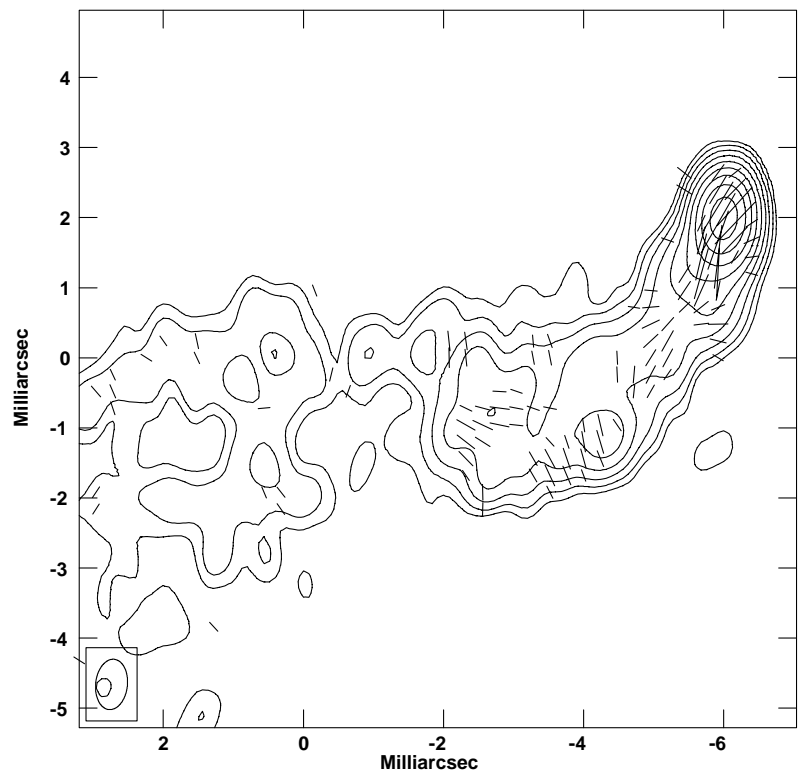

Figure 4.9: VLBA observations of $\mathrm{Mkn} 501$ at $15 \mathrm{GHz}$ in $\mathrm{Au}-$ gust 15, 1997, taken from Marscher 1999. Contour levels are $-0.25,0.25,0.5,1,2,4,8,16,32$ and $64 \%$ of the peak intensity of $0.44 \mathrm{Jy}_{\text {beam }}{ }^{-1}$ while the scale of the polarization electric vectors is $0.0078 \mathrm{Jy}_{\mathrm{beam}}{ }^{-1} \mathrm{mas}^{-1}$. The magnetic field is perpendicular to the jet near the axis and parallel to the jet along the boundaries. A strong bend at about 3 mas from the core, which at a distance of $160 \mathrm{Mpc}$ (for a Hubble constant of $65 \mathrm{~km} \mathrm{~s}^{-1} \mathrm{Mpc}^{-1}$ ) corresponds to a length of $\sim 2.3 \mathrm{pc}$ (i.e. 1 mas $\simeq 0.78$ pc), is clearly visible.

al. 1999). The unification of BL Lacs and Fanaroff-Riley (FR) I objects (see sect. 1.4) may add another piece of evidence to such a jet configuration: in order to account for the observed spectral properties an at least two-fold jet velocity structure seems to be required in which a fast spine is surrounded by a slow (but still relativistic) layer (Chiaberge et al. 2000). Support for such a possibility is now positively provided by numerical jet simulations (cf. Aloy et al. 2000; Frank et al. 2000).

If the observed periodical flux variability results primarily from doppler boosting, the relative amplitude depends on the spectral index [e.g. Eq. (4.2)]. Hence, for a $\mathrm{TeV}$ flux ratio between minimum and maximum of $\sim 8$, the X-ray flux ratio should be $\sim(5-7)$ applying a hard X-ray spectral index $\alpha \simeq 0.6-0.9$ (cf. Lamer \& Wagner 1998; Pian et al. 1998) and 
the corresponding shift in the break frequency (i.e. $\Delta \nu_{\mathrm{obs}}=\Delta \delta \nu$ ) should be given by a factor of $\sim(1.5-1.6)$. Such values seem to be consistent with observations made by the BeppoSAX X-ray astronomy satellite (Pian et al. 1998) and may also be recovered, using a broken power law fit, in RXTE observations of Mkn 501 (cf. Krawczynski et al. 2000, their Figs. 1 and 2a). Gamma-ray observations carried out by the Cherenkov Array at Themis (CAT) imaging telescope, also reveal a shift of the maximum peak energy apparently in accordance with the expectation above (Djannati-Atai et al. 1999). Small changes in the maximum electron Lorentz factor or the magnetic field along the trajectory of the emission region may further add to flux variations. If there is indeed an additional flux contribution, e.g. low energy emission from the layer, a stationary component comparable to the observed infrared-optical flux (e.g. Pian et al. 1998; cf. also Kataoka et al. 1999) or an additional component responsible for the soft X-ray emission (e.g. Lamer \& Wagner 1998; Wagner et al. 1999), the amplitude of the Doppler modulation may decrease to lower frequencies.

In the above presentation, an influence of jet precession due to gravitomagnetic and geodetic origin (e.g. Thorne et al. 1986) has not been considered so far. For the geodetic precession due to the gravitational field of the companion $M$ we have $\Omega_{\text {geo }}=3 G M^{2} \Omega_{k} /\left[2(m+M) c^{2} d\right]$ while the gravitomagnetic precession due to the motion of the companion is given by $\Omega_{\mathrm{gm}}=2 G m M \Omega_{k} /\left[(m+M) c^{2} d\right]$, with $\Omega_{k}$ the keplerian angular frequency and $d$ the separation. Thus, the total precessional angular frequency $\Omega_{p}$ may be written as

$$
\Omega_{p}=\Omega_{\mathrm{geo}}+\Omega_{\mathrm{gm}}=\frac{(3 m+\mu)}{2 d} \frac{G}{c^{2}} \Omega_{k},
$$

where $\mu$ denotes the reduced mass. Hence, using appropriate values, the total precessional period is determined by

$$
P_{p}=\frac{2 \pi}{\Omega_{p}} \simeq 580 \times d_{16}^{5 / 2}\left(M_{8}+m_{8}\right)^{1 / 2} \frac{1}{m_{8}} \frac{1}{M_{8}} \frac{1}{(1+3 M / 4 m)} \mathrm{yrs} .
$$

Since this driving period is much larger than the orbital period (cf. Table 4.1, 4.2), a precessional modulation should be negligible during a few revolutions. Interestingly, a precessional period of $\sim 10^{4}$ yrs agrees with the driving frequency found by Conway \& Wrobel (1995) in order to explain the misalignment of the radio jet in Mkn 501 on parsec and kiloparsec scale (see also Villata \& Raiteri 1999).

If the binary hypothesis is correct, the observable period should remain similar during different outburst phases unless there is a change in the general jet properties. For example, an increase in the observed period should then 
be accompanied by a decrease in the bulk Lorentz factor or, on larger time scales, by an increase of the inclination angle due to the jet precession [cf. also Eq. 4.7. 


\section{Chapter 5}

\section{Summary}

There is increasing observational and theoretical evidence for jet rotation in AGN. In this thesis we have considered the effects of jet rotation by dividing them into intrinsic and extrinsic caused phenomena. The former ones have been related to rotation of matter inside the jet while the latter ones describe the rotation of the whole jet due to an external cause (e.g. in a binary system).

In the first part the centrifugal acceleration of particles at the base of an (intrinsically) rotating jet magnetosphere has been considered. In MHD scenarios such rotating magnetospheres are usually regarded as the essential ingredient required to produce relativistic outflow velocities and to account for the observed well-collimated jets. Following a test particle approach, the equation for the radial acceleration has been derived and solved using analytical methods. We have found that for particles moving outwards along rotating magnetic field lines, the energy gain is limited in particular by the breakdown of the bead-on-the-wire approximation which occurs in the vicinity of the light cylinder $r_{\mathrm{L}}$. This has not been taken into account up to now. The corresponding upper limit for the maximum Lorentz factor $\gamma_{\max }$ for electrons scales $\propto B^{2 / 3} r_{\mathrm{L}}^{2 / 3}$, with $B$ the magnetic field strength at $r_{\mathrm{L}}$, and is at most of an order of $10^{2}-10^{3}$ for the conditions regarded to be typical for BL Lac objects. Additionally it has been found, that inclusion of inverse-Compton losses in a disk radiation field which approaches the Eddington limit results in a decreasing upper bound for the maximum Lorentz factor. Our results show that centrifugal acceleration may provide pre-accelerated particles which are required for efficient Fermi-type particle acceleration mechanisms on larger scales in radio-jets. It has been further pointed out that inverse-Compton scattering of accretion disk photons 
by centrifugally accelerated electrons may be of particular relevance by contributing to the observed $10-100 \mathrm{keV}$ X-ray humps in AGN typically attributed to Compton reflection.

The study of particle acceleration has then been extended in the second part. Following previous work by Webb (1989) and Webb, Jokipii \& Morfill (1994), but incorporating velocity profiles more relevant to AGN jets, steady state solutions of the kinetic equation describing the transport of energetic particles in a collisionless, (intrinsically) rotating background flow have been investigated. Our analysis utilizes a simple version of the (mixed-frame) relativistic transport equation derived in the diffusion approximation. For this approximation to be valid, the scattering of energetic particles by magnetic field irregularities carried in the background flow is required to occur rapidly enough to allow for nearly isotropy of the particle distribution in the comoving fluid frame.

By considering rigid and keplerian rotation profiles, Green's solutions for the (comoving) steady-state distribution $f_{0}^{\prime}\left(r, p^{\prime}\right)$ are derived in a basic jet model where the plasma moves along the jet axis at relativistic $v_{z}$ while the velocity component in the azimuthal direction is purely azimuthal and characterized by its angular frequency $\Omega$. Particle acceleration then generally occurs as a consequence of centrifugal and/or shear effects. In the special case of rigid rotation, shearing in the background flow is absent and energy changes are due solely to the centrifugal force. Hence the energy gain is found to be analogous to that in the bead-on-the-wire approach presented in part 1. Additionally, the acceleration efficiency is shown to be essentially dependent on the form of the diffusion coefficient. If one considers for example, a simple momentum-dependence for the mean time interval $\tau_{c}$ between collisions, i.e. $\tau_{c} \propto p^{\alpha}$, the efficiency is enhanced for $\alpha<0$ and reduced for $\alpha>0$. In the case of keplerian rotation on the other hand, both shear and centrifugal effects are present and the transport equation becomes quite more complex. However, for non-relativistic rotation where the shear effects eventually dominate, solutions of the transport equation have been found using analytical techniques which are likely to be appropriate for keplerian flows in the outermost part of the jet. Most interestingly, a powerlaw-type particle momentum spectrum $f_{0}\left(r, p^{\prime}\right) \propto p^{-(3+\alpha)}$ is recovered in the case where the collision time (only) depends on momentum as $\tau_{c} \propto p^{\alpha}$, with $\alpha>0$, in agreement with the results derived by Berezkho \& Krymskii (1981). Besides providing the possibility for a detailed inclusion of centrifugal effects, the derivation presented here has the additional advantage to allow of a radial dependence of the scattering time $\tau_{c}$ which 
could result in a slightly steepening of the momentum distribution (if $\tau_{c}$ is a decreasing function of the radial distance from the jet axis).

It is pointed out that the acceleration of particles by rotating and shearing jet flows could thus be of important relevance for the interpretation of powerlaw-like high energy spectra. This seems particularly interesting in view of recent observational evidence for extended acceleration processes in AGN jets (e.g. in the optical jet of $3 \mathrm{C} 273$ ).

Occasionally, observational evidence for intrinsic jet rotation, i.e. for rotation of material inside the jet, seems not unambiguous. For, the rotation of jets due to an extrinsic cause, e.g. the precession of a jet due to the gravitational torque or the rotation of a jet in a binary system, might result in a similar observational effect. Modelling of intrinsic and and extrinsic jet rotation might thus be important in order to discern the real cause. As an illustrative example thus, the extrinsic caused jet rotation in a binary black hole system has been investigated in the last part of this thesis. It is shown that the observed $\mathrm{TeV}$ and X-ray periodicity of $\sim 23$ days, measured during a flaring state in the BL Lac object Mkn 501, could be basically interpreted as a doppler-shifted flux modulation due to the orbital motion of the relativistic jet. Using typical jet properties inferred from emission models, we find an intrinsic orbital period of $(6-14)$ yrs and a centre-of-mass distance of $(2-3.5) \times 10^{16} \mathrm{~cm}$. These results indicate a binary system which is very close. Upper limits on the allowed binary masses have been derived assuming the binary separation to be of an order for which gravitational radiation becomes dominant. We find a primary mass of $\sim 10^{8} M_{\odot}$ and a corresponding maximum allowed secondary mass in the range of $\sim(1-9) \times 10^{7} M_{\odot}$ if gas accretion occurs on around the salpeter time scale. The derived mass ranges interestingly agree with the expectations from merger scenarios. Further observational evidences and implications have been disussed. If confirmed by further observations, Mkn 501 will be the first object where $\gamma$-ray astronomy notedly contributes to the increasing evidence for binary black hole systems. 


\section{Appendix A}

\section{On the Hamiltonian for a bead on a rigidly rotating wire}

\section{A.1 Non-relativistic limit}

We may gain some insights into the physical comprehension of centrifugal acceleration by first considering the motion of a bead on a rigidly rotating wire in the non-relativistic limit. Let $m_{0}$ be the mass of a bead on a wire where the wire rotates rigidly with uniform angular velocity $\Omega$ in the $x-$ $y$-plane. Then there are two constraints on the particle motion, first, a so-called "holonomic-scleronomic" (i.e. $z=0$ ) and secondly, a so-called "holonomic-rheonomic" constraint (time-dependent, $y=x \tan \Omega t$ ). Hence,

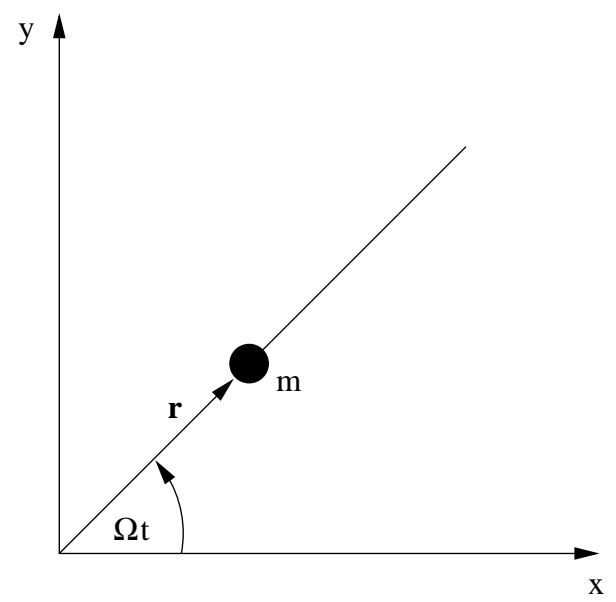

one may describe the motion of the bead by one generalised co-ordinate, an 
appropriate choice being the co-ordinate $r$. The Lagrangian $L$ for the bead is given by the kinetic energy term $T$

$$
L=T=\frac{1}{2} m_{0}\left(\dot{r}^{2}+r^{2} \Omega^{2}\right) .
$$

Since $L$ is not explicitly time-dependent (i.e. $\partial L / \partial t=0$ ), Noether's theorem implies, that the Hamiltonian $H$ is a constant of motion. Using the generalised momentum $P$

$$
P=\frac{\partial L}{\partial \dot{r}}=m_{0} \dot{r}
$$

the Hamiltonian is given by

$$
H=\dot{r} P-L=\frac{1}{2} m_{0}\left(\dot{r}^{2}-r^{2} \Omega^{2}\right) .
$$

To be sure, this Hamiltonian is not identical with the overall energy of the bead in the inertial, non-rotating reference frame [cf. Eq. (A.1)]. In contrast to Newtonian mechanics, constraint forces are eliminated in Lagrangian mechanics (cf. d'Alembert's principle). Thus, the work actually done by a constraint force will not appear in the Hamiltonian. Although this does not make any difference for scleronomic constraints, it does for rheonomic constraints. Hence the homogeneity of time only implies conservation of the overall energy for holonomic-scleronomic, conservative systems. Taking the difference of Eq. (A.3) and Eq. (A.1) one gets $H-L=-m_{0} \Omega^{2} r^{2}$, which is two times the centrifugal potential energy. Accordingly, the Hamiltonian $H$ may be interpreted as the conserved energy of the bead in the noninertial, rigidly rotating frame of reference. The rotation then simply adds a centrifugal potential energy term $-0.5 m_{0} \Omega^{2} r^{2}$ to the kinetic energy in the rotating frame $0.5 m_{0} \dot{r}^{2}$. Changing from the inertial reference frame $K$ at rest to the noninertial, rigidly rotating reference frame $K^{\prime}$, the energy of the bead is not the same but consequently transforms like (see also Landau \& Lifshitz, p.128)

$$
E^{\prime}=E-\Omega^{2} r^{2}
$$

where $E^{\prime}$ denotes the energy in $K^{\prime}$ and $E=T$ the energy in $K$.

\section{A.2 Relativistic case}

Consider now the case of a relativistically moving wire. Using the principle of least action, the Lagrangian of the bead with rest mass $m_{0}$ may be written as

$$
L=-m_{0} c^{2} \sqrt{1-\Omega^{2} r^{2} / c^{2}-\dot{r}^{2} / c^{2}},
$$


where the action integral is defined by

$$
I=-m_{0} c \int_{a}^{b} d s=\int_{t_{a}}^{t_{b}} L d t
$$

with $d s^{2}$ being the line element and where for an arbitrary but chosen inertial reference frame $d s=c d \tau=\sqrt{1-\vec{v}^{2} / c^{2}} d t$ with $\vec{v}=d \vec{x} / d t$. Here, the Lagrangian is not identical with the energy of the bead in the inertial frame $K$ which, using cylindrical co-ordinates, is given by the zero component of the four momentum (multiplied by $c$ )

$$
\left(p^{\alpha}\right)=\gamma m_{0}(c, \dot{r}, \Omega, 0) \quad \text { with } \quad \gamma=\frac{1}{\sqrt{1-\Omega^{2} r^{2} / c^{2}-\dot{r}^{2} / c^{2}}},
$$

and metric $\left(g_{\alpha \beta}\right)=\operatorname{diag}\left(-1,1, r^{2}, 1\right)$. By using Eq. (A.5), the generalised momentum $P$ may be written as

$$
P=\frac{\partial L}{\partial \dot{r}}=\frac{m_{0} \dot{r}}{\sqrt{1-\Omega^{2} r^{2} / c^{2}-\dot{r}^{2} / c^{2}}} .
$$

Now, since $L$ is not explicitly time-dependent, the Hamiltonian $H$ is a constant of motion (cf. Noether's theorem)

$$
H=\dot{r} P-L=\frac{m_{0} c^{2}\left(1-\Omega^{2} r^{2} / c^{2}\right)}{\sqrt{1-\Omega^{2} r^{2} / c^{2}-\dot{r}^{2} / c^{2}}}=\text { const. }
$$

For an interpretation of this expression, let us consider the transformation of the particle four momentum from the laboratory frame $K$ to a local Lorentz frame $K^{\prime}$ moving with the rotating wire (which is not identical with the comoving frame of the particle). Transformations from one Lorentz frame to another are given by $V^{\prime \alpha}=\Lambda_{\beta}^{\alpha} V^{\beta}$ with $\left(\Lambda_{\beta}^{\alpha}\right)$ the relevant Lorentz matrix. For a general representation one has $\Lambda_{\alpha}^{0}=-u_{\alpha} / c$, where $u_{\alpha}$ denotes the covariant components of the four velocity of wire in the laboratory frame $K$. Hence, one immediately arrives at the following transformation for the zero component of the (contravariant) four momentum

$$
p^{\prime 0}=-p^{\alpha} u_{\alpha} / c \quad \text { with } \quad u_{\alpha}=\gamma_{l}\left(-c, 0, \Omega r^{2}, 0\right),
$$

where the normalization is given by $\gamma_{l}=1 / \sqrt{1-\Omega^{2} r^{2} / c^{2}}$. Eq. (A.10), multiplied by $c$, may be identified with the energy of the bead in the comoving frame $K^{\prime}$, where the energy is given as a function of quantities measured in the laboratory frame. Using Eq. (A.7) one easily finds

$$
p^{\prime 0}=m_{0} c \frac{\sqrt{1-\Omega^{2} r^{2} / c^{2}}}{\sqrt{1-\Omega^{2} r^{2} / c^{2}-\dot{r}^{2} / c^{2}}} .
$$


Thus, the Hamiltonian could be written as

$$
H=p^{\prime 0} c / \gamma_{l} \text {. }
$$

Here, the additional gamma factor $\gamma_{l}$ takes into account that time is measured in the laboratory frame $K$ (where $d \tau=\sqrt{1-\Omega^{2} r^{2} / c^{2}} d t$ ). However, if we transform to the momentary rest frame of the rotating wire (where $d \tau=d t$ ), this additional gamma factor vanishes. Hence, in analogy to nonrelativistic mechanics, the Hamiltonian $H$ may be interpreted as the energy of the bead in the momentary rest frame of the rotating wire (cf. Machabeli \& Rogava 1994). Then, for small velocities $H$ reduces to

$$
H=m_{0} c^{2}+\frac{1}{2} m_{0} \dot{r}^{2}-\frac{1}{2} m_{0} \Omega^{2} r^{2},
$$

which (neglecting a rest mass energy term) is indeed the same as Eq. (A.3). 


\section{Appendix B}

\section{Derivation of the \\ non-relativistic diffusive \\ particle transport equation}

By following the work of Earl, Jokipii and Morfill 1988, we derive in this appendix the equation for the transport of energetic particles in a nonrelativistic, collisionless plasma background flow which explicitly includes for example, energy changes due to the flow shear. In the course of this analysis the background plasma is treated as collisionless (i.e. mutual interactions amongst the particles are neglected) while the energetic particles are assumed to be scattered and driven near to isotropy by small-scale magnetic field irregularities carried in the background flow. The simple derivation presented here utilizes the comoving particle momentum and may thus give insights into the use of a mixed-frame transport equation providing a valuable background for the (quite more extensive) relativistic treatment.

Now, denote by $\vec{p} \equiv m \vec{w}, m$ being the relativistic particle mass, the particle momentum in the laboratory frame (the lab. frame) where the background plasma is in motion with velocity $u_{i}$. The time-evolution of the phase-space particle distribution function in the lab. frame is governed by the Boltzmann equation

$$
\frac{\partial f}{\partial t}+w_{i} \frac{\partial f}{\partial x_{i}}+F_{i} \frac{\partial f}{\partial p_{i}}=\left(\frac{\partial f}{\partial t}\right)_{\mathrm{S}},
$$

where $f \equiv f(\vec{x}, \vec{p}, t)$ denotes the particle distribution function, $F_{i}$ is the external force and the term on the right hand side describes the scattering by magnetic field irregularities.

Since the electric field vanishes in the plasma rest frame (i.e. the comoving frame), the magnitude of the particle momentum is conserved in that frame 
and thus, in a first approach the scattering in the comoving frame may be modelled using a simple BKG-type collision term of the form

$$
\left(\frac{\partial f}{\partial t}\right)_{\mathrm{S}}=(f-<f>) / \tau
$$

where the relaxation time $\tau$ could be a function of momentum. As one would like to keep this simple form of the scattering operator, it proves useful to use a mixed system of phase-space coordinates in Eq. (B.1) such that quantities which are operated upon the scattering operator, i.e. the momentum, are evaluated in the comoving frame while the time and space coordinates are still measured in the lab. frame (for details see, for example Kirk, Melrose, Priest 1994, p. 248):

Now, for non-relativistic flow speeds the components $p_{i}^{\prime}$ of the particle momentum in the plasma rest frame (i.e. the comoving frame) are related to those in the lab. frame by a simple Galilean transformation

$$
p_{i}=m w_{i}=p_{i}^{\prime}+m u_{i} .
$$

Hence, to the lowest order the space derivative transforms in detail like

$$
\begin{aligned}
\left(\frac{\partial f(\vec{x}, \vec{p}, t)}{\partial x_{i}}\right)_{\vec{p}} & =\left(\frac{\partial f\left(\vec{x}, \vec{p}^{\prime}, t\right)}{\partial x_{i}}\right)_{\vec{p}^{\prime}}+\left(\frac{\partial p_{j}^{\prime}}{\partial x_{i}}\right)_{\vec{p}} \frac{\partial f\left(\vec{x}, \vec{p}^{\prime}, t\right)}{\partial p_{j}^{\prime}} \\
& =\frac{\partial f\left(\vec{x}, \vec{p}^{\prime}, t\right)}{\partial x_{i}}-m \frac{\partial u_{j}}{\partial x_{i}} \frac{\partial f\left(\vec{x}, \vec{p}^{\prime}, t\right)}{\partial p_{j}^{\prime}}
\end{aligned}
$$

where $\left(\partial f / \partial x_{i}\right)_{\vec{p}}$ means a derivative at constant $\vec{p}$. A similar transformation holds for the time-derivative. Substituting these expressions in Eq. (B.1), we obtain the following equation

$$
\frac{\partial f}{\partial t}+\left(u_{i}+\frac{p_{i}^{\prime}}{m}\right) \frac{\partial f}{\partial x_{i}}+\left[F_{i}-m\left(\frac{\partial u_{i}}{\partial t}+u_{j} \frac{\partial u_{i}}{\partial x_{j}}\right)-p_{j}^{\prime} \frac{\partial u_{i}}{\partial x_{j}}\right] \frac{\partial f}{\partial p_{i}^{\prime}}=\left(\frac{\partial f}{\partial t}\right)_{\mathrm{S}} .
$$

Consider now the case in which the external force vanishes $\left(F_{i}=0\right)$. In the diffusion approximation, which is applied here, the distribution function is assumed to be almost isotropic, the departure from isotropy being small. Thus the distribution function may be written as $f=f_{0}+f_{1}$, with $f_{0} \equiv f_{0}\left(\vec{x}, p^{\prime}, t\right)$ being the isotropic and $f_{1}$ being the anisotropic part of the distribution function, i.e. $\langle f\rangle=f_{0}$ and $\left\langle f_{1}\right\rangle=0$ when averaged over solid angle. By inserting this approximation in Eq. (B.4) and averaging over solid angle we arrive at

$$
\frac{\partial f_{0}}{\partial t}+u_{i} \frac{\partial f_{0}}{\partial x_{i}}+\left\langle\frac{p_{i}^{\prime}}{m} \frac{\partial f_{1}}{\partial x_{i}}\right\rangle-m\left(\frac{\partial u_{i}}{\partial t}+u_{j} \frac{\partial u_{i}}{\partial x_{j}}\right)\left\langle\frac{\partial f_{1}}{\partial p_{i}^{\prime}}\right\rangle
$$




$$
-\frac{\partial u_{i}}{\partial x_{j}}\left(\left\langle p_{j}^{\prime} \frac{\partial f_{0}}{\partial p_{i}^{\prime}}\right\rangle+\left\langle p_{j}^{\prime} \frac{\partial f_{1}}{\partial p_{i}^{\prime}}\right\rangle\right)=0
$$

For this diffusion approximation to be valid, one requires the phase, space and time derivatives of $f_{1}$ being small compared to the corresponding derivatives of $f_{0}$. Hence, by subtracting Eq. (B.5) from Eq. (B.4), the anisotropic part $f_{1}$ of the distribution function may be approximated in terms of $f_{0}$

$$
\begin{aligned}
f_{1} & \simeq \tau\left(-\frac{p_{k}^{\prime}}{m} \frac{\partial f_{0}}{\partial x_{k}}+m\left[\frac{\partial u_{k}}{\partial t}+u_{l} \frac{\partial u_{k}}{\partial x_{l}}\right] \frac{p_{k}^{\prime}}{p^{\prime}} \frac{\partial f_{0}}{\partial p^{\prime}}\right) \\
& +\tau\left(p_{l}^{\prime} \frac{\partial u_{k}}{\partial x_{l}} \frac{p_{k}^{\prime}}{p^{\prime}} \frac{\partial f_{0}}{\partial p^{\prime}}-\frac{\partial u_{k}}{\partial x_{l}}<p_{k}^{\prime} p_{l}^{\prime}>\frac{1}{p^{\prime}} \frac{\partial f_{0}}{\partial p^{\prime}}\right),
\end{aligned}
$$

where we have used the relation $\partial f_{0} / \partial p_{k}^{\prime}=\left(\partial p^{\prime} / \partial p_{k}^{\prime}\right) \partial f_{0} / \partial p^{\prime}=$ $\left(p_{k}^{\prime} / p^{\prime}\right) \partial f_{0} / \partial p^{\prime}$

If we insert Eq. (B.6) into Eq. (B.5), we get the full non-relativistic transport equation. Note, that averaging over solid angle yields the following relations

$$
\begin{aligned}
<p_{i}^{\prime}> & =0 \\
<p_{i}^{\prime} p_{j}^{\prime}> & =\frac{1}{3} p^{\prime 2} \delta_{i j} \\
<p_{i}^{\prime a} p_{j}^{\prime b}> & =0 \text { for } a+b=3 \\
<p_{i}^{\prime 2} p_{j}^{\prime 2}> & =\frac{1}{15} p^{\prime 4} \text { for } \mathrm{i} \neq \mathrm{j} \\
<p_{i}^{\prime 4}> & =\frac{1}{5} p^{\prime 4}
\end{aligned}
$$

which may be easily verified by using spherical coordinates ( $\phi$ for the Colatitude, $\theta$ for the azimuth) and the definition of solid angle $\Omega=\int \sin \phi \mathrm{d} \phi \mathrm{d} \theta$ equal to $4 \pi$ if integrated over all space.

Neglecting in Eq. (B.5) all terms which are proportional to $\left(\partial u_{i} / \partial t\right)\left(\partial u_{i} / \partial t\right)$, $u_{j}\left(\partial u_{i} / \partial x_{j}\right)\left(\partial u_{i} / \partial t\right)$ and $u_{j}\left(\partial u_{i} / \partial x_{j}\right) u_{l}\left(\partial u_{i} / \partial x_{l}\right)$ we arrive at:

$$
\begin{aligned}
\frac{\partial f_{0}}{\partial t} & +u_{i} \frac{\partial f_{0}}{\partial x_{i}}-\frac{1}{3} \frac{\partial u_{i}}{\partial x_{i}} p^{\prime} \frac{\partial f_{0}}{\partial p^{\prime}}-\frac{\tau}{m^{2}}<p_{i}^{\prime} p_{k}^{\prime}>\frac{\partial^{2} f_{0}}{\partial x_{i} \partial x_{k}} \\
& +\tau\left[\frac{\partial^{2} u_{k}}{\partial t \partial x_{i}}+\frac{\partial u_{l}}{\partial x_{i}} \frac{\partial u_{k}}{\partial x_{l}}+u_{l} \frac{\partial^{2} u_{k}}{\partial x_{i} \partial x_{l}}\right] \frac{p_{i}^{\prime} p_{k}^{\prime}>\frac{\partial f_{0}}{p^{\prime}}}{\partial p^{\prime}} \\
& +\tau\left[\frac{\partial u_{k}}{\partial t}+u_{l} \frac{u_{k}}{x_{l}}\right] \frac{p_{i}^{\prime} p_{k}^{\prime}>\frac{\partial^{2} f_{0}}{p^{\prime}}}{\partial p^{\prime} \partial x_{i}} \\
& +\tau\left[\frac{\partial u_{i}}{\partial t}+u_{j} \frac{\partial u_{k}}{\partial x_{j}}\right]\left(\frac{\partial f_{0}}{\partial x_{i}}+\frac{<p_{i}^{\prime} p_{k}^{\prime}>}{p^{\prime}} \frac{\partial^{2} f_{0}}{\partial x_{k} \partial p^{\prime}}+\frac{<p_{i}^{\prime} p_{k}^{\prime}>\frac{\partial f_{0}}{p^{\prime}}}{\partial x_{k}}\right)
\end{aligned}
$$




$$
\begin{aligned}
& -\tau \frac{\partial u_{i}}{\partial x_{j}} \frac{<p_{j}^{\prime} p_{k}^{\prime}>}{p^{\prime}} \frac{\partial u_{k}}{\partial x_{i}} \frac{\partial f_{0}}{\partial p^{\prime}}-\tau \frac{\partial u_{i}}{\partial x_{j}} \frac{<p_{j}^{\prime} p_{l}^{\prime}>}{p^{\prime}} \frac{\partial u_{i}}{\partial x_{l}} \frac{\partial f_{0}}{\partial p^{\prime}} \\
& +\tau \frac{\partial u_{i}}{\partial x_{j}} \frac{\partial u_{k}}{\partial x_{l}} \frac{<p_{j}^{\prime} p_{l}^{\prime} p_{i}^{\prime} p_{k}^{\prime}>}{p^{\prime 3}} \frac{\partial f_{0}}{\partial p^{\prime}}-\tau \frac{\partial u_{i}}{\partial x_{j}} \frac{\partial u_{k}}{\partial x_{l}} \frac{<p_{j}^{\prime} p_{l}^{\prime} p_{i}^{\prime} p_{k}^{\prime}>\frac{\partial^{2} f_{0}}{p^{\prime 3}}}{\partial p^{\prime 2}} \\
& +\frac{1}{3} \tau \frac{\partial u_{i}}{\partial x_{j}} \frac{\partial u_{k}}{\partial x_{k}} \frac{<p_{j}^{\prime} p_{i}^{\prime}>}{p^{\prime}} \frac{\partial f_{0}}{\partial p^{\prime}}+\frac{1}{3} \tau \frac{\partial u_{i}}{\partial x_{j}} \frac{\partial u_{k}}{\partial x_{k}}<p_{j}^{\prime} p_{i}^{\prime}>\frac{\partial^{2} f_{0}}{\partial p^{\prime 2}} \\
& -\frac{\partial \tau}{\partial p^{\prime}} \frac{\partial u_{i}}{\partial x_{j}} \frac{\partial u_{k}}{\partial x_{l}} \frac{<p_{j}^{\prime} p_{l}^{\prime} p_{i}^{\prime} p_{k}^{\prime}>}{p^{\prime 2}} \frac{\partial f_{0}}{\partial p^{\prime}}+\frac{1}{3} \frac{\partial \tau}{\partial p^{\prime}} \frac{\partial u_{i}}{\partial x_{j}} \frac{\partial u_{k}}{\partial x_{k}}<p_{i}^{\prime} p_{j}^{\prime}>\frac{\partial f_{0}}{\partial p^{\prime}}=0 .
\end{aligned}
$$

Using the relations for the mean values above [e.g. Eq. (B.7)], especially:

$$
\begin{aligned}
\frac{\partial u_{i}}{\partial x_{j}} \frac{\partial u_{k}}{\partial x_{l}}<p_{j}^{\prime} p_{l}^{\prime} p_{i}^{\prime} p_{k}^{\prime}> & =\frac{\partial u_{i}}{\partial x_{k}} \frac{\partial u_{i}}{\partial x_{k}}<p_{k}^{\prime} p_{k}^{\prime} p_{i}^{\prime} p_{i}^{\prime}> \\
& +\frac{\partial u_{i}}{\partial x_{i}} \frac{\partial u_{k}}{\partial x_{k}}<p_{i}^{\prime} p_{k}^{\prime} p_{k}^{\prime} p_{i}^{\prime}> \\
& +\frac{\partial u_{i}}{\partial x_{k}} \frac{\partial u_{k}}{\partial x_{i}}<p_{k}^{\prime} p_{i}^{\prime} p_{k}^{\prime} p_{i}^{\prime}>
\end{aligned}
$$

and collecting the appropriate terms together, the full non-relativistic transport equation may be written in the following form:

$$
\begin{aligned}
\frac{\partial f_{0}}{\partial t}+u_{i} \frac{\partial f_{0}}{\partial x_{i}} & -\frac{p^{\prime}}{3} \frac{\partial u_{i}}{\partial x_{i}} \frac{\partial f_{0}}{\partial p^{\prime}}-\frac{\partial}{\partial x_{i}}\left(\frac{\tau}{3} \frac{p^{2}}{m^{2}} \frac{\partial f_{0}}{\partial x_{i}}\right) \\
& +\frac{2 \tau p^{\prime}}{3} A_{i} \frac{\partial^{2} f_{0}}{\partial x_{i} \partial p^{\prime}}+\frac{1}{3 p^{\prime 2}} \frac{\partial\left(\tau p^{\prime 3}\right)}{\partial p^{\prime}} A_{i} \frac{\partial f_{0}}{\partial x_{i}} \\
& -\frac{\Gamma}{p^{\prime 2}} \frac{\partial}{\partial p^{\prime}}\left(\tau p^{\prime 4} \frac{\partial f_{0}}{\partial p^{\prime}}\right)+\frac{p^{\prime}}{3} \frac{\partial\left(\tau A_{i}\right)}{\partial x_{i}} \frac{\partial f_{0}}{\partial p^{\prime}}=0
\end{aligned}
$$

where the new quantities are defined by:

$$
A_{i}=\frac{\partial u_{i}}{\partial t}+u_{l} \frac{\partial u_{i}}{\partial x_{l}}
$$

and

$$
\Gamma=\frac{1}{30}\left(\frac{\partial u_{i}}{\partial x_{k}}+\frac{\partial u_{k}}{\partial x_{i}}\right)^{2}-\frac{2}{45} \frac{\partial u_{i}}{\partial x_{i}} \frac{\partial u_{k}}{\partial x_{k}} .
$$

In the special case of quasi-steady flows, the first term on the right hand side of Eq. (B.11) might be neglected. In addition, for non-relativistic flow speeds 
we have $p^{2} / m^{2} \simeq w^{2}$. Inserting these results, Eq. (B.10) reduces to Eq. (6) given in Earl, Jokipii and Morfill 1988. As already stated by Earl et al., the second, third and fourth term in Eq. (B.10) describe the well-known effects of convection, adiabatic energy change and diffusion. The terms involving $A_{i}$ describe the effects of inertial drifts while the term involving $\Gamma$ includes energy changes due to the shear and the divergence of the flow and might be related to the viscosity of the background flow.

It should be noted that the present derivation did not consider the influence of an average background magnetic field on the particle transport. As was shown by Williams and Jokipii 1991, the inclusion of a magnetic field may lead both to an anisotropic viscosity and a reduction of the magnitude of the isotropic viscosity. 


\section{References}

[1] Aaron S.E., 1999, in: Takalo L.O., Sillanpää A. (eds.) BL Lac Phenomenon. PASP Conf. Ser. 159, p. 427

[2] Abraham Z., Carrara E.A., 1998, ApJ 496, 172

[3] Abraham Z., Romero G.E., 1999, A\&\&A 344, 61

[4] Abramowicz M.A., 1990, MNRAS 245, 733

[5] Abramowicz M.A., Prasanna A.R., 1990, MNRAS 245, 720

[6] Abramowitz M., Stegun I.A. (eds.), 1965, Handbook of Mathematical Functions, Dover Publications, New York

[7] Achterberg A., Krülls W.M., 1992, A\&A 265, L13

[8] Aharonian F., Akhperjanian A.G., Barrio J.A., et al. 1999, A\&A 349, 29

[9] Aloy M.-A., Gómez J.-L., Ibáñez J.-M., et al. 2000, ApJ 528, L85

[10] Antonucci R., 1993, ARA \& A 31, 473

[11] Appl S., Camenzind M., 1993, A\&A 270, 71

[12] Appl S., Sol H., Vicente L., 1996, A\&A 310, 419

[13] Artymowicz P., 1998, in: Abramowicz M.A. et al. (eds.) Theory of Black Hole Accretion Disks. Cambridge Univ. Press, Cambridge, p. 202

[14] Attridge J.M., Roberts D.H., Wardle J.F.C., 1999, ApJ 518, L87

[15] Axford W.I., Leer E., Skadron A., 1977, in: Proc. of 15th International Cosmic Ray Conference (Plodiv), vol. 11, p. 132

[16] Bade N., Beckmann V., Douglas N.G., et al. 1998, A ש̇A 334, 459

[17] Bahcall J.N., Kirhakos S., Schneider D.P., 1994, ApJ 435, L11

[18] Bahcall J.N., Kirhakos S., Schneider D.P., et al. 1995, ApJ 452, L91

[19] Ball L., Kirk J.G., 1992, ApJ 396, L39

[20] Ball L., Kirk J.G., 1995, A $\& A$ 303, L57 
[21] Barnes J.E., Hernquist L., 1992, ARA \& A 30, 705

[22] Barnes J.E., Hernquist L., 1996, ApJ 471, 115

[23] Bateman H., 1964, Partial Differential Equations of Mathematical Physics, Cambridge Univ. Press, Cambridge

[24] Bednarek W., Kirk J.G., Mastichiadis A., 1996, A $\mathscr{G} A$ 307, L17

[25] Begelman M.C., 1994, in: The Nature of Compact Objects in Active Galactic Nuclei, eds. A. Robinson, R. Terlevich, Cambridge Univ. Press, Cambridge, p. 361

[26] Begelman M.C., Blandford R.D., Rees M.J., 1980, Nature 287, 307

[27] Begelman M.C., Blandford R.D., Rees M.J., 1984, Rev. Mod. Phys. 56 255

[28] Bell A.R., 1978, MNRAS 182, 147

[29] Berezhko E.G., 1981, JETP Letters 33, 399

[30] Berezhko E.G., 1982a, Geomagnetizm i Aeronomiia 22, 353

[31] Berezhko E.G., 1982b, Sov. Astr. Lett. 8, 403

[32] Berezhko E.G., 1984, Advances in Space Research 4, 405

[33] Berezhko E.G., Krymskii G.F., 1981, Sov. Astr. Lett. 7, 352

[34] Berezhko E.G., Krymskii G.F., 1982, Izvestiia AN SSSR, Seriia Fiz. 46, 1656

[35] Beskin V.S., Rafikov R.R., 2000, MNRAS 313, 433

[36] Bhatnagar P.L., Gross E.P., Krook M., 1954, Phys. Rev. 94, 511

[37] Biretta J.A., 1993, in: Astrophysical Jets, eds. D. Burgarella, M. Livio, C.P. O'Dea, Cambridge Univ. Press, Cambridge, p. 263

[38] Blandford R.D., 1990, in: Blandford R.D., Netzer H., Woltjer L, Active Galactic Nuclei, (Saas Fee 20). Springer: Berlin, p. 161

[39] Blandford R.D., 1993, in: Astrophysical Jets, eds. D. Burgarella, M. Livio, C.P. O'Dea, Cambridge Univ. Press, Cambridge, p. 15

[40] Blandford R.D., Rees M.J., 1974, MNRAS 169, 395

[41] Blandford R.D., McKee C.F., Rees M.J., 1977, Nature 267, 211

[42] Blandford R.D., Znajek R.L., 1977, MNRAS 179, 433

[43] Blandford R.D., Ostriker J.P., 1978, ApJ 221, 29

[44] Blandford R.D., Königl A., 1979, ApJ 232, 34

[45] Blandford R.D., Payne D.G., 1982, MNRAS 199, 883 
[46] Blandford R.D., Netzer H., Woltjer L., 1990, Active Galactic Nuclei (Saas-Fee Advanced Course 20 Lecture Notes), eds. T.J.-L. Courvoisier, M. Mayor, Springer: Berlin

[47] Britzen S., Witzel A., Krichbaum T.P., et al. 2000, $A \& A$ 360, 65

[48] Brotherton M.S., Gregg M.D., Becker R.K., et al. 1999, ApJ 514, L61

[49] Browne I.W.A., Jackson N., 1992, in: Physics of Active Galactic Nuclei, eds. W.J. Duschl, S.J. Wagner. Springer, Berlin, p. 628

[50] Buchholz H., 1953, Die konfluente hypergeometrische Funktion (Ergebnisse der angewandten Mathematik ; 2), Springer: Berlin

[51] Camenzind M., 1986, A\&A 156, 137

[52] Camenzind M., 1989, in: Accretion Disks and Magnetic Fields in Astrophysics, ed. G. Belvedere, Kluwer, Dordrecht, p. 129

[53] Camenzind M., 1995, in: Reviews in Modern Astronomy 8, ed. G. Klare, Hamburg, p. 201

[54] Camenzind M., 1996, in: Solar and Astrophysical Magnetohydrodynamic Flows, ed. K.C. Tsinganos, Kluwer, Dordrecht, p. 699

[55] Camenzind M., Krockenberger M., 1992, A\&A 255, 59

[56] Cao X., 1997, MNRAS 291, 145

[57] Catanese M., 1999, in: Takalo L.O., Sillanpää A. (eds.) BL Lac Phenomenon. PASP Conf. Ser. 159

[58] Catanese M., Bradbury S.M., Breslin A.C., et al. 1997, ApJ 487, L143

[59] Catanese M., Weekes T.C., 1999, PASP 111, 1193

[60] Catanese M., Sambruna R.M., 2000, ApJ 534, L39

[61] Cavaliere A., Malquori D., 1999, ApJ 516, L9

[62] Cecil G., Wilson A.S., Tully R.B., 1992, ApJ 390, 365

[63] Celotti A., Fabian A.C., Rees M.J., 1998, MNRAS 293, 239

[64] Celotti A., Blandford R.D., 2000, in: Proc. ESO Workshop on "Black Holes in Binaries and Galactic Nuclei", Garching (Sept. 1999), eds. L. Kaper, E.P.J. van den Heuvel, P.A. Woudt, Springer: Berlin, to appear (astro-ph/0001056)

[65] Chandrasekhar S., 1943, ApJ 97, 255

[66] Chedia O.V., Kahniashvili T.A., Machabeli G.Z., Nanobashvili I.S., 1996, Ap\&SSS 239, 57 
[67] Chiaberge M., Celotti A., Capetti A., Ghisellini G., 2000, A\&A 358, 104

[68] Contopoulos I, Kazanas D., Fendt C., 1999, ApJ 511, 351

[69] Conway J.E., Wrobel J.M., 1995, ApJ 439, 98

[70] Dermer C.D., Schlickeiser R., 1992, Science 257, 1642

[71] Dermer C.D., Schlickeiser R., Mastichiadis A., 1992, A $\&$ A 256, L27

[72] Djannati-Atai A., Piron F., Barrau A., et al. 1999, A $\mathscr{G} A$ 350, 17

[73] Dondi L., Ghisellini G., 1995, MNRAS 273, 583

[74] Drury L.O'C., 1983, Rep. Prog. Phys. 46, 973

[75] Duffy P., Ball L., Kirk J.G., 1995, ApJ 447, 364

[76] Duschl W.J., Wagner S.J. (Eds.), 1992, Physics of Active Galactic Nuclei, Spinger: Berlin

[77] Earl J.A., Jokipii J.R., Morfill G., 1988 ApJ 331, L91

[78] Eckart A., Genzel R., 1996, Nature 383, 415

[79] Eracleous M., Halpern J.P., Gilbert A.M., et al. 1997, ApJ 490, 216

[80] Falcke H., Biermann P.L., Duschl W.J., Mezger P.G., 1993, A\&A 270, 102

[81] Falcke H., Biermann P.L., 1995, A\&A 293, 665

[82] Fan J.H., Xie G.Z., Bacon R., 1999, A\&AS 136, 13

[83] Fanaroff B.L., Riley J.M., 1974, MNRAS 167, 31P

[84] Fendt C., 1997a, A\&A 319, 1025

[85] Fendt C., 1997b, A\&A 323, 999

[86] Fendt C., Memola E., 2001, A\&A 365, 631

[87] Fermi E., 1949, Phys. Rev 75, 1169

[88] Fermi E., 1954, ApJ 119, 1

[89] Frank A., Lery T., Gardiner T.A., et al. 2000, ApJ 540, 342

[90] Frank J., King A., Raine D., 1995, Accretion Power in Astrophysics, (second edition), Cambridge Univ. Press: Cambridge

[91] Fritze v.-Alvensleben U., 1996, in: Leitherer C. et al. (eds) From Stars to Galaxies. ASP Conf. Ser. 98, p. 496

[92] Gangadhara R.T., 1996, A\& A 314, 853

[93] Gangadhara R.T., Lesch H., 1997, A\&A 323, L45 
[94] Gaskell C.M., 1996a, in: Jets from Stars and Galactic Nuclei (Lecture Notes in Physics, vol. 471), ed. W. Kundt. Springer, Berlin, p. 165

[95] Gaskell C.M., 1996b, ApJ 464, L107

[96] Gebhardt K., Bender R., Bower G., et al. 2000, ApJ 539, L13

[97] Genzel R., Pichon C., Eckart A., Gerhard O.E., Ott T., 2000, MNRAS 317, 348

[98] George I.M., Fabian A.C., 1991, MNRAS 249, 352

[99] Giovannini G., Taylor G.B., Arbizzani E., et al. 1999, ApJ 522, 101

[100] Gold T., 1968, Nature 218, 731

[101] Gold T., 1969, Nature 221, 25

[102] Ghosh K.K., Ramsey B.D., Sadun A.C., Soundararajaperumal S., 2000, ApJS 127, 11

[103] Gould A., Rix, H-W., 2000, ApJ 532, L29

[104] Guilbert P.W., Rees M.J., 1988, MNRAS 233, 475

[105] Haehnelt M.G., Natarajan P., Rees M., 1998, MNRAS 300, 817

[106] Hanasz M., Sol H., Sauty C., 2000, MNRAS 316, 494

[107] Hayashida N., Hirasawa H., Ishikawa F., et al. 1998, ApJ 504, L71

[108] Heidt J., 1999, in: Takalo L.O., Sillanpää A. (eds.) BL Lac Phenomenon. PASP Conf. Ser. 159, p. 367

[109] Henriksen R.N., Rayburn D.R., 1971, MNRAS 152, 323

[110] Hillas A.M., 1999, Astropart. Phys. 11, 27

[111] Ho L.C., 1998, in: Chakrabarti S.K. (ed.) Observational Evidence for Black Holes in the Universe. Kluwer, Dordrecht, p. 157

[112] Ivanov P.B., Papaloizou J.C.B., Polnarev A.G., 1999, MNRAS 307, 79

[113] Iyer S., Prasanna A.R., 1993, Class. Q. Grav. 10, L13

[114] Jester S., Röser H.-J., Meisenheimer K., et al. 2001, in: Particles and Fields in Radio Galaxies, eds. R.A. Laing, K.M. Blundell, (ASP Conf. Ser.), to appear (astro-ph/0011413)

[115] Jokipii J.R., Morfill G.E., 1990, ApJ 356, 255

[116] Kaastra J.S., Roos N., 1992, A\&A 254, 96

[117] Kahniashvili T.A., Machabeli G.Z., Nanobashvili I.S., 1997, Physics of Plasmas 4, 1132

[118] Kamke E., 1944, Differentialgleichungen, Leibzig 
[119] Kanbach G., 1996, in: Proc. of the Heidelberg Workshop on GammaRay Emitting AGN, eds. J.G. Kirk, M. Camenzind, C. v. Montigny, S. Wagner, Heidelberg, October 1996 (MPI H-V37-1996), p.1

[120] Kataoka J., Mattox J.R., Quinn J., et al. 1999, ApJ 514, 138

[121] Katz J.I., 1991, ApJ 367, 407

[122] Katz J.I., 1997, ApJ 478, 527

[123] Kauffmann G., 1996, MNRAS 281, 487

[124] Kellermann K.I., Pauliny-Toth I.I.K., 1969, it ApJ 155, L71

[125] Kellermann K.I., Sramek R., Schmidt M., et al., 1989, AJ98, 1195

[126] Khachikian E.Y., Weedman D.W., 1974, ApJ 192, 581

[127] Khanna R., Camenzind M., 1996, A\&A 307, 665

[128] Kirk J.G., Schlickeiser R., Schneider P., 1988, ApJ 328, 269

[129] Kirk J.G., Melrose D.B., Priest E.R., 1994, Plasma Astrophysics, eds. A.O. Benz, T.J.-L. Couvoisier, Springer (Berlin)

[130] Kirk J.G., Rieger F.M., Mastichiadis A., 1998, A $\mathscr{G} A$ 333, 452

[131] Kirk J.G., Duffy P., 1999, J. Phys. G: Nucl. Part. Phys. 25, R163

[132] Kochanek C.S., Falco E.E., Muñoz J.A., 1999, ApJ 510, 590

[133] Konopelko A., 1999, Astropart. Phys. 11, 135

[134] Kormendy J., Richstone D., 1995, ARA\&A 33, 581

[135] Kranich D., deJager O.C., Kestel M., et al. 1999, in: Proc. of 26th International Cosmic Ray Conference (Salt Lake City), vol. 3, p. 358

[136] Krawczynski H., Coppi, P.S., Maccarone T., Aharonian F.A., 2000, A $\because A$ 353, 97

[137] Krymskii G.F., 1977, Akademiia Nauk SSSR, Doklady 234, 1306

[138] Kunieda H., Awaki H., Koyama K., et al. 1990, Nature 345, 786

[139] Lamer G., Wagner S.J., 1998, A $\& A$ 331, L13

[140] Landau L.D., Lifshitz E.M., 1960, Mechanics, Pergamon Press, Oxford

[141] Lesch H., Birk G.T., 1997, A\&A,324, 461

[142] Lehto H.J., Valtonen M.J., 1996, ApJ 460, 207

[143] Lery T., Frank A., 2000, ApJ 533, 897

[144] Lesch H., Appl S., Camenzind M., 1989, A\&A 225, 341

[145] Levinson A., 1992, ApJ 401, 73 
[146] Lightman A.P., White T.R., 1988, ApJ 335, 57

[147] Lindquist R.W., 1966, Ann.Phys. 37, 487

[148] Livio M., 1999, Physics Reports 311, 225

[149] Lynden-Bell D., 1969, Nature 223, 690

[150] Macchetto F., Marconi A., Axon D.J., et al. 1997, ApJ 489, 579

[151] Machabeli G.Z., Rogava A.D., 1994, Phys. Rev. A 50, 98

[152] Magorrian J., Tremaine S., Richstone D., et al. 1998, AJ 115, 2285

[153] Mannheim K., 1993, A\&A 269, 67

[154] Mannheim K., 1997, in: XXXIInd Recontres des Moriond, (Les Arcs, Savoie, France), eds. Y. Girand-Héraud, J. Tran Thanh Van, Editions Frontieres, p. 17

[155] Mannheim K., 1998, Science 279, 684

[156] Mannheim K., Krülls W.M., Biermann P.L., 1991, A $\& A$ 251, 723

[157] Mannheim K., Westerhoff S., Meyer H., Fink H.-H., 1996, A\&\&A 315, 77

[158] Maraschi L., Ghisellini G., Celotti A., 1992, ApJ 397, L5

[159] Marcowith A., Henri G., Pelletier G., 1995, MNRAS 277, 681

[160] Marscher A.P., 1999, Astropart. Phys. 11, 19

[161] Marscher A.P., Marchenko-Jorstad S.G., Mattox J.R., et al. 2000, in: Astrophysical Phenomena Revealed by Space VLBI, eds. H. Hirabayashi, P.G. Edwards and D.W. Murphy, published by the Institute of Space and Astronautical Science, p. 39.

[162] Mastichiadis A., Kirk J.G., 1997, A $6 A$ 320, 19

[163] Meisenheimer K., Röser H.-J, Schlötelburg M., 1996, A\&A 307, 61

[164] Meisenheimer K., Yates M.G., Röser H.-J., 1997, A\& A 325, 57

[165] Melia F., Falcke H., 2001, ARA \& A 39, to appear

[166] Melrose D.B., 1980, Plasma Astrophysics, Volume 2, Gordon \& Breach: New York, p. 105

[167] Melrose D.B., 1994, in: Kirk J.G., Melrose D.B., Priest E.R., 1994, Plasma Astrophysics, eds. A.O. Benz, T.J.-L. Couvoisier, Springer (Berlin), p. 113

[168] Merritt D., 1999, in: Galaxy Dynamics from the Early Universe to the Present, eds. F. Combes, G. Mamon, V. Charmandaris, ASP Conf. Ser. 197, 221 
[169] Mihalas D., Mihalas B.W., 1984, Foundations of Radiation Hydrodynamics, Oxford University Press, Oxford

[170] Misner C.W., Thorne K.S., Wheeler J.A., 1973, Gravitation, Freeman: San Francisco.

[171] Miyoshi M., Moran J., Herrnstein J., et al. 1995, Nature 373, 127

[172] Morse P.M., Feshbach H., 1953, Methods of Theoretical Physics, Vol. I, McGraw-Hill Book Company: New York.

[173] Mortlock D.J., Webster R.L., Francis P.J., 2000, MNRAS, in press

[174] Narayan R., 1997, in: Proc. IAU Symp. 188, The Hot Universe, p. 41

[175] Narayan R., Yi I., 1994, ApJ 428, L13

[176] Netzer H., 1990, in: Blandford R.D., Netzer H., Woltjer L, Active Galactic Nuclei, (Saas Fee 20). Springer: Berlin, p. 57

[177] Nilsson K., Pursimo T., Takalo L.O., et al. 1999, PASP 111, 1223

[178] Nishikawa D., Hayashi S., Chamoto N., et al. 1999, in: Proc. of 26th International Cosmic Ray Conference (Salt Lake City), vol. 3, p. 354

[179] Osterbrock D.E., 1981, ApJ 249, 462

[180] Ostriker J.P., Tremaine S.D., 1975, ApJ 202, L113

[181] Ostrowski M., 1990, A\& A 238, 435

[182] Ostrowski M., 1999, in: Ostrowski M., Schlickeiser R. (eds), Plasma Turbulence and Energetic Particles in Astrophysics (Proc. Internat. Conf. Cracow), Obserwatorium Astronomiczne, Uniwersytet Jagiellonski, Kraków 1999, p. 256

[183] Owen F.N., Odea C.P., Inoue M, Eilek J.A., 1985, ApJ 294, L85

[184] Padovani P., 1999, in: High Energy Emission from AGN and Unified Schemes, Proc. of Vulcano Workshop 1998 'Frontier Objects in Astrophysics and Particle Physics', (astro-ph/9901130)

[185] Parker E.N., 1965, Planet. Space Sci. 13, 9

[186] Perlman E.S., Biretta J.A., Zhou F., et al. 1999, AJ 117, 2185

[187] Peterson B., 1997, An introduction to active galactic nuclei, Cambridge Univ. Press, Cambridge

[188] Petry D., Böttcher M., Connaughton V., et al. 2000, ApJ 536, 742

[189] Pian E., Vacanti G., Tagliaferri G., et al. 1998, ApJ 492, L17

[190] Pohl M., Schlickeiser R., 2000, A\&广A 354, 395 
[191] Polnarev A.G., Rees M., 1994, A\&A 283, 301

[192] Protheroe R.J., Bhat C.L., Fleury P., et al. 1998, in: Proc. 25th International Cosmic Ray Conference (Durban), vol. 8, p.317

[193] Quinn J., Bond I.H., Boyle P.J., et al. 1999, ApJ 518, 693

[194] Rees M.J., 1966, Nature 211, 468

[195] Rees M.J., 1984, ARA \& A 22, 471

[196] Rees M.J., 1994, in: Muñoz-Tuñón C. \& Sánchez F. (eds.) The Formation and Evolution of Galaxies. Cambridge Univ. Press, Cambridge, p. 503

[197] Richstone D, 1998, in: Folkner W.M. (ed.) Laser Interferometer Space Antenna. AIP Conf. Proc. 456, p. 41

[198] Richstone D., Ajhar E.A., Bender R., et al. 1998, Nature 395A, 14

[199] Rieger F.M. 1997, Diplomarbeit (M.Sc. thesis), Ruprecht-KarlsUniversität Heidelberg

[200] Rieger F.M., Kirk J.G., Mastichiadis A., 1999, in: Büchner J., et al. (eds.) Plasma Astrophysics and Space Physics, Kluwer: Dordrecht, p.699

[201] Rieger F.M., Mannheim K., 1999, in: Büchner J., et al. (eds.) Plasma Astrophysics and Space Physics, Kluwer: Dordrecht, p.705

[202] Rieger F.M., Mannheim K., 2000a, A $\mathscr{\mho} A$ 353, 473

[203] Rieger F.M., Mannheim K., 2000b, A\&A 359, 948

[204] Rieger F.M., Mannheim K., 2001a, in: Aharonian F.A., Völk H. (eds) High Energy Gamma-Ray Astronomy, AIP Conf. Proc., in press

[205] Rieger F.M., Mannheim K., 2001b, in: Aharonian F.A., Völk H. (eds) High Energy Gamma-Ray Astronomy, AIP Conf. Proc., in press

[206] Riffert H., 1986, ApJ 310, 729

[207] Roland J, Teyssier R., Roos N., 1994, A\&A 290, 357

[208] Roos N., 1988, ApJ 334, 95

[209] Roos N., Kaastra J.S., Hummel C.A., 1993, ApJ 409, 130

[210] Rybicki G.B., Lightman A.P., 1979, Radiative Processes in Astrophysics, Wiley, New York

[211] Salucci P., Ratnam C., Monaco P., Danese L., 2000, MNRAS 317, 488

[212] Samuelson F.W., Biller S.D., Bond I.H., et al. 1998, ApJ 501, L17 
[213] Scarpa R., Urry C.M., Falomo R., et al. 1999, ApJ 521, 134

[214] Schlickeiser R., 1989, ApJ 336, 243

[215] Schlickeiser R., 1996, A\&AS 120, 481

[216] Schmidt M., 1963, Nature 197, 1040

[217] Schramm K.-J., Borgeest U., Camenzind M., et al. 1993, A\&A 278, 391

[218] Seyfert C., 1943, ApJ 97, 28

[219] Sikora M., Begelman M.C., Rees M.J., 1994, ApJ 421, 153

[220] Sillanpää A., Haarala S., Valtonen M.J., et al. 1988, ApJ 325, 628

[221] Skilling J., 1975, MNRAS 172, 557

[222] Sol H., Pelletier G., Asséo E., 1989, MNRAS 237, 411

[223] Sonego S., Massar M, 1996, MNRAS 281, 659

[224] Spada M., 1999, Astropart. Phys. 11, 59

[225] Spada M., Salvati M., Pacini F., 1999, ApJ 511, 136

[226] Sparks W.B., Biretta J.A., Macchetto F., 1996, ApJ 473, 254

[227] Spruit H.C., 1994, in: Cosmical Magnetism, ed. D. Lynden-Bell, Kluwer: Dordrecht, p. 33

[228] Spruit H.C., Foglizzo, Stehle R., 1997, MNRAS 288, 333

[229] Stewart J., 1971, Nonequilibrium Relativistic Kinetic Theory, Springer: Berlin

[230] Stickel M., Fried J.W., Kühr H., 1993, A\&AS 98, 393

[231] Subramanian P., Becker P.A., Kazanas D., 1999, ApJ 523, 203

[232] Swain M.R., Bridle A.H., et al. 1998, ApJ 507, L29

[233] Tanaka Y., Nandra K., Fabian A.C., et al. 1995, Nature 375, 659

[234] Taub A.H.,1967, Relativistic Hydrodynamics, in: Ehlers J. (ed.), Relativity Theory and Astrophysics. 1. Relativity and Cosmology. American Mathematical Society, Providence, Rhode Island (Lectures in Applied Mathematics, Vol. 8), p. 170

[235] Thorne K.S., Price R.H., Macdonald D.A. (eds), 1986, Black Holes: The Membrane Paradigm. Yale Univ. Press

[236] Tölke F., 1967, Praktische Funktionenlehre, Bd.3., Springer: Berlin.

[237] Toomre A., Toomre J., 1972, ApJ 178, 623 
[238] Urry M.C., Padovani P., 1995, PASP 107, 803

[239] Villata M., Raiteri C.M., Sillanpää A., Takalo L.O., 1998, MNRAS 293, L13

[240] Villata M., Raiteri C.M., 1999, A\&\&A 347, 30

[241] Wagner S.J., Lamer G., Bicknell G. V., 1999, Astronom. Nach. 320, 226

[242] Wagner S.J., Witzel A., 1995, ARA \& A 33, 163

[243] Wandel A., Peterson B.M., Malkan M.A., 1999, ApJ 526, 579

[244] Webb G.M., 1985, ApJ 296, 319

[245] Webb G.M., 1989, ApJ 340, 1112

[246] Webb G.M., 1992, in: Particle acceleration in cosmic plasmas, AIP Conf. Proc. 264, New York, p.287

[247] Webb G.M., Jokipii J.R., Morfill G.E., 1993, in: Proc. 23rd Internat. Cosmic Ray Conf., Calgary, Canada 2, 271

[248] Webb G.M., Jokipii J.R., Morfill G.E., 1994, ApJ 424, 158

[249] White S.D.M., 1997, in: Börner G., Gottlober S. (eds.) The Evolution of the Universe. Report of the Dahlem Workshop. Berlin 1995, p. 227

[250] Williams L.L, Jokipii J.R., 1991, ApJ 371, 639

[251] Williams L.L, Jokipii J.R., 1993, ApJ 417, 725

[252] Wolfram S., 1996, The Mathematica book. Cambridge Univ. Press: Cambridge

[253] Woltjer L., 1990, in: Blandford R.D., Netzer H., Woltjer L, Active Galactic Nuclei, (Saas Fee 20). Springer: Berlin, p. 1

[254] Zeldovič Ya.B., Novikov I.D., 1964, Sov. Phys. Dokl. 158, 811 



\section{Acknowledgements}

I am greatly indebted to several people for giving support and encouragement during the process of this thesis:

In particular, I have benefited very much from the inspiring discussions with my supervisor Karl Mannheim. He has shared with me his fascination for new astrophysical themes, introduced me to the art of writing papers and encouraged me in following up some of my own ideas. I would like to thank him very much for his patience, interest and responsibility.

Furthermore I would like to thank Prof. K. Beuermann for his immediate readiness to write the additional report on this thesis.

I am also indebted to various people (V. Beskin, R. Buras, J.H. Fan, R.T. Gangadhara, J. Katz, H. Lesch) whose interests, comments or responses on my published results have contributed to this thesis in its final form.

I am really grateful for the atmosphere and the help I have experienced in our high energy astrophysics group. My sincere gratitude is to Klaus Beuermann, Boris Gänsicke, Rick Hessman, Klaus Reinsch, Bettina Hoffmann, Jens Kube and all the others who shared my everyday life at the Institute. I especially would like to thank those sharing and enjoying the room with me: Andreas Fischer (for all his support during the beginnings), Christian Hettlage (also for his assistance in the Mathematica calculations), Tanja Kneiske (also for her checking the references) and Matthias Schreiber (also for providing the latex-macro and for pleasant chat) - it was a nice time!

I would like to thank as well many of my friends, especially from the German IFES group for discussions about God, world and human life, opening my eyes up to a life much richer than mere theoretical calculations.

Last but not least I would like to thank my family for their patience and confidence. In particular, I deeply would like to thank my wife Damaris for all her love and encouragement throughout these years. She has closely shared with me the phases of enthusiasm and disappointment during all stages of this work.

Finally, support of this work by the Deutsche Forschungsgemeinschaft (MA $1545 / 2-1)$ is gratefully acknowledged. 



\section{List of Publications}

\section{Refereed Journals}

Kirk J.G., Rieger F.M., Mastichiadis A., 1998, Particle acceleration and synchrotron emission in blazar jets, $A \mathscr{E} A \mathbf{3 3 3}, 452$

Rieger F.M., Mannheim K., 2000a, Particle acceleration by rotating magnetospheres in active galactic nuclei, $A \& A$ 353, 473

Rieger F.M., Mannheim K., 2000b, Implications of a possible 23 day periodicity for binary black hole models in Mkn 501, A\& A 359, 948

\section{Conference proceedings}

Rieger F.M., Kirk J.G., 1997, Particle acceleration and synchrotron emission at shocks in relativistic jets, in $A G$-Abstract Series 13, 66

Rieger F.M., Mannheim K., 1998, Test particle acceleration due to centrifugal force in AGN, in AG-Abstract Series 14, 67

Kirk J.G., Rieger F.M., Mastichiadis A., 1999, The Variability Patterns of Synchrotron Emission and Particle Acceleration in Blazars, in: Takalo L.O., Sillanpää A. (eds.), BL-Lac Phenomenon, ASP Conference Series Nr. 159, p. 325

Rieger F.M., Kirk J.G., Mastichiadis A., 1999, On the nonthermal Xray emission in blazar jets, in: Büchner J., et al. (eds.) Plasma Astrophysics and Space Physics, Kluwer: Dordrecht, p.699 
Rieger F.M., Mannheim K., 1999, On the quasi-periodical oscillations in Mkn 501, in AG-Abstract Series 15, 63

Rieger F.M., Mannheim K., 1999, Centrifugal acceleration in jets from AGN, in: Büchner J., et al. (eds.) Plasma Astrophysics and Space Physics, Kluwer: Dordrecht, p.705

Rieger F.M., Mannheim K., 2001a, Test particle acceleration by rotating jet magnetospheres, in: Aharonian F.A., Völk H. (eds.) High Energy Gamma-Ray Astronomy, AIP Conf. Proc. 558, 827

Rieger F.M., Mannheim K., 2001b, A possible black hole binary in Mkn 501, in: Aharonian F.A., Völk H. (eds.) High Energy Gamma-Ray Astronomy, AIP Conf. Proc. 558, 716 


\section{Curriculum Vitae}

Name

Born

Nationality

Marital status

\section{Education}

Sept. 1978 - July 1982

Sept. 1982 - June 1991

13.06.1991

Oct. 1991 - March 1997

16.07.1993

25.03 .1997

since July 1997
Frank Michael Rieger

November 22, 1971 in Pforzheim

(Baden-Württemberg)

German

married to Damaris Rieger, born Dürr

Schiller Grundschule, Mühlacker

Theodor-Heuss-Gymnasium, Mühlacker

Acquisition of the German matriculation standard, Abitur

Studies of physics at the Ruprecht-Karls-

Universität Heidelberg

Vordiplom

Diploma in physics with the thesis

Teilchenbeschleunigung und Synchrotronemission in Blazar-Jets, supervisor:

John G. Kirk (MPI für Kernphysik, Heidelberg)

$\mathrm{PhD}$ studies in astrophysics at

the Universitäts-Sternwarte Göttingen,

supervisor: Karl Mannheim

Memberships

1996 -

$1997-$

$1998-$
Deutsche Physikalische Gesellschaft

(German Physical Society)

Deutsche Astronomische Gesellschaft

(German Astronomical Society)

British Society for the Philosophy of Science 